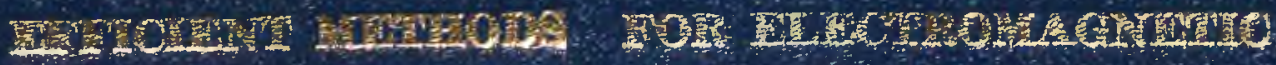

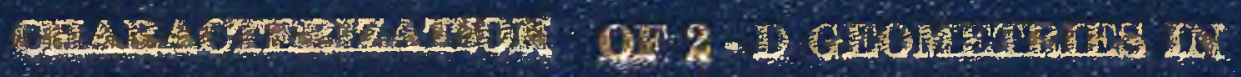

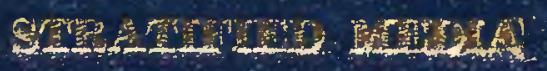

\title{
25 TFESSIS
}

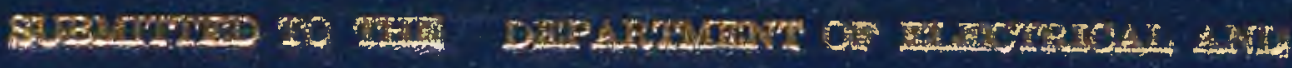

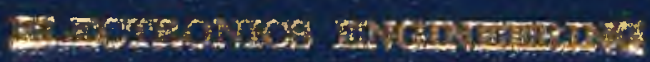

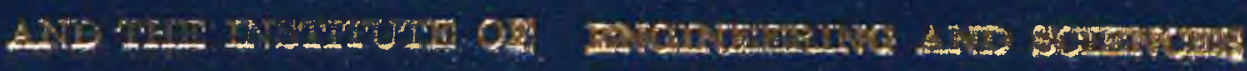

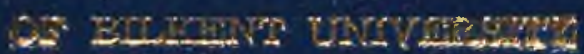

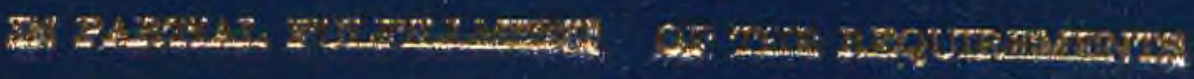

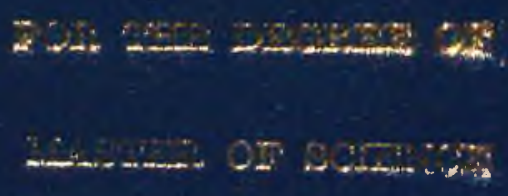

3..

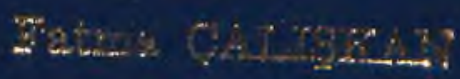

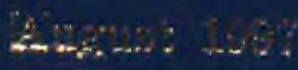




\title{
EFFICIENT METHODS FOR ELECTROMAGNETIC CHARACTERIZATION OF 2-D GEOMETRIES IN STRATIFIED MEDIA
}

\author{
A THESIS \\ SUBMITTED TO THE DEPARTMENT OF ELECTRICAL AND \\ ELECTRONICS ENGINEERING \\ AND THE INSTITUTE OF ENGINEERING AND SCIENCES \\ OF BILKENT UNIVERSITY \\ IN PARTIAL FULFILLMENT OF THE REQUIREMENTS \\ FOR THE DEGREE OF \\ MASTER OF SCIENCE \\ Fatma Galiskan. \\ $\mathrm{By}$
}

Fatma Çalışkan

August 1997 
$Q C$

661

. C 35

1997

B. 
I certify that I have read this thesis and that in my opinion it is fully adequate, in scope and in quality, as a thesis for the degree of Master of Science.

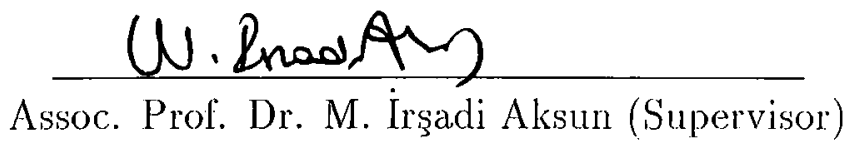

I certify that I have read this thesis and that in my opinion it is fully adequate, in scope and in quality, as a thesis for the degree of Master of Science.

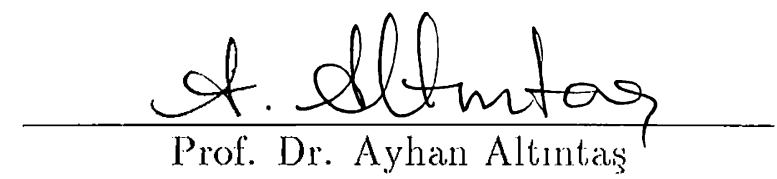

I certily that I have read this thesis and that in my opinion it, is lully adcouate, in scope and in quality, as a thesis for the degrce of Master of Science.

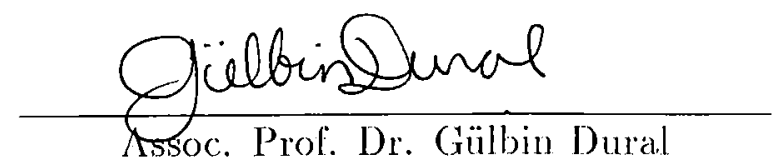

Approved for the Institute of Engineering and Sciences:

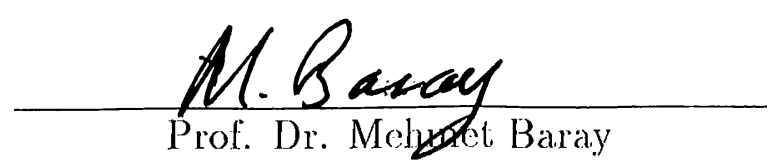

Director of Institute of Engineering and Sciences 


\title{
ABSTRACT \\ EFFICIENT METHODS FOR ELECTROMAGNETIC CHARACTERIZATION OF 2-D GEOMETRIES IN STRATIFIED MEDIA
}

\author{
Fatma Çalışkan \\ M.S. in Electrical and Electronics Engineering \\ Supervisor: Assoc. Prof. Dr. M. İrşadi Aksun \\ August 1997
}

\begin{abstract}
Numerically efficient method of moments (MoM) algorithms are developed for and applied to 2-D geometries in mult ilayer media. These are, namely. the spatialdomain MoM in conjunction with the closed-form Green's functions. the spectraldomain MoM using the generalized pencil of functions (GPOF) algorithm and a FFT algorithm to evaluate the MoM matrix entries. These approaches are mainly to improve the computational efficiency of the evaluation of the MoM matrix entries. Among these, the spectral-domain Moll using the GPOF algorithm is the most efficient approach for printed multilayer geometries. The assessment of the efficiency of this method is performed on several problems. by comparing the matrix fill times for these three approaches. In addition a new iterative algorithm
\end{abstract}


is developed to solve the MoM matrix equation, which is based on dividing a large object into subregions and solving the matrix equation on each subregion by considering the effects of other regions. This iterative algorithm is applied to some large geometries and is compared to a traditional $\mathrm{Ll}$ decomposition algorithm for the assessment of its numerical efficiency. It is observed that the iterative algorithm is numerically more efficient as compared to the $\mathrm{LU}$ decomposition.

Keyu:ords : Method of moments, Planarly layered media, Green's functions, Iterative method 


\title{
ÖZET
}

\section{ÇOK KATMANLI ORTAMLARDA 2 BOYUTLU GEOMETRILERIN ELEKTROMANYETIK TANIMLAMASI IÇİ ETKILI YÖNTEMLER}

\author{
Fatma Çalıskan \\ Elektrik ve Elektronik Mühendisliḡi Bölümü Yühsek Lisans \\ Tez Yöneticisi: Doç. Dr. M. İrşadi Aksun
}

Ag̃ustos 1997

Cok katmalı ortamlarda 2 boyutlu geometriler için saỵıal olarak etkin moment metodu (MoM) algoritmaları geliştirildi ve 2 boyutlu geometrilere uygulandı. Mo.I matris elemanlarım hesaplamak için kullanılan গöntemler. kapalı formda Green fonksiyonları ile birlikte kullanılan gerçek uzay moment metodu. generalized pencil of functions (GPOF) algoritması kullanılarak yapılan spektral uzay moment metodu ve moment metodu matris elemanlarm hesaplamak için hızlı Fourier dönüşümü (FFT) algoritması kullanılmasıdır. Bu yöntemler moment metodu matris elemanların işlemsel olarak verimli bir şekilde bulmak işin kullamrlar. Bu yöntemler işinde sok tabakalı basılı geometriler işin en etkili olan GPOF algoritması kullamlarak yapilan spektral uzạ moment metodudur. Bu 
metodun etkili olduğu bir çok problem üzerinde ü̧ metodun matris elemanlarını hesaplama süreleri karşılaştırılarak gösterildi. Buna ek olarak moment metodu matris denklemini çözmek için yeni yinelemeli bir algoritma geliştirildi. Bu yōntem büyük bir nesneyi alt bölgelere parçalayıp, her bölgenin matris denklemini diğer bölgelerin etkilerini dūşūnerek hesaplamaktadır. Bu algoritma bazı büyük geometrilere uygulandı ve sayısal etkisini değerlendirmek için geleneksel bir yöntem olan LU ayrıştırma algoritması ile karşılaştırıldı. Sonuç olarak. yinelemeli algoritmanın LU decomposition algoritmasından sayısal olarak daha etkili olduğu gözlendi.

Anahtar Kelimeler: Moment metodu, Düzlemsel cok katmanlı ortam. Green fonksiyonlari, Yinelemeli yöntem 


\section{ACKNOWLEDGEMENT}

I would like to express my deepest gratitude to Assoc. Prof. Dr. M. Irşadi Aksun and Asst. Prof. Dr. Levent Gürel for their supervision and encouragement in all steps of the development of this work.

I would like to thank Prof. Dr. Aỵhan Altıntaş and Assoc. Prof. Dr. Gülbin Dural for reading and commenting on the thesis and for the honor they gave me by presiding the jury. I would also like to thank Prof. Dr. Erdem Yazgan and Asst. Prof. Dr. Adnan Köksal for supporting me during my undergraduate studies.

Many thanks to Noyan. Ertem, Tolga, Siğdem. Deniz. Kürşat. Karim. Ạ. han and Tuns for their valuable help. Sincere thanks are also extended to my officemates Erdem, Fehmi and Moez. I also thank all my friends for their moral support.

It is a pleasure to express my special thanks to my family. especially to my mother, father and Zeynep. for their endless love. patience, support and encouragement. 
To my mother, father and Zeynep

viii 


\section{Contents}

1 Introduction 1

2 Green's Functions in Spectral and Spatial Domains 4

2.1 Green`s Functions in the Spectral Domain 6

2.2 Closed-Form Green's Functions in the Spatial Domain . . . . . . 11

3 Formulation of Electric Field Integral Equation in 2-D 21

3.1 Method of Moments ................. 22

3.2 Electric Field in $2-\mathrm{D} \ldots \ldots \ldots \ldots . \ldots . \ldots . \ldots . \ldots$

3.3 Basis and Testing Functions ............... 2 i

3.4 MoM Formulation in Spatial Domain . . . . . . . . . . . . 30

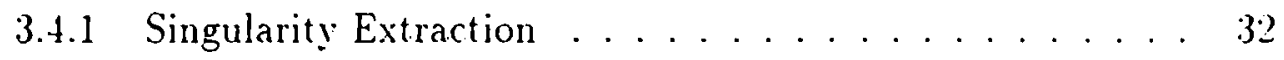

3.5 MoM Formulation using FFT . . . . . . . . . . . . . 34

3.6 MoM Formulation in Spectral Domain 39

4 Numerical Examples $\quad \mathbf{4 2}$

4.1 Examples for the MoM Formulation in the Spatial Domain . . . . 43

4.1 .1 A Single Horizontal Strip . . . . . . . . . . . . . . 43

4.1.2 Analytical Solution of Cylinders in a Homogeneous Medium AS 
4.1.3 A Conducting Cylinder in an Inhomogeneous Medium . . 5:3

4.1.4 A Rectangular Cylinder in an Inhomogeneous Medium . . 55

4.2 Comparison of the Methods . . . . . . . . . . 57

4.3 Examples for the MoM Formulation in the Spectral Domain . . 64

5 Solving Linear Equations by the Iterative Method 73

5.1 Comparison of the Iterative Method and LU Decomposition 76

5.1 .1 Example 1.................... 76

5.1 .2 Example 2..................... 87

5.1 .3 Example $3 \ldots \ldots \ldots . \ldots . \ldots . \ldots 9$

$\begin{array}{llr}6 & \text { Conclusions } & 96\end{array}$ 


\section{List of Figures}

2.1 Sources embedded in a multilayer medium. . . . . . . . .

2.2 Definition of the Sommerfeld integration path, and the paths $C_{a p 1}$ and $C_{a p 2}$ used in two-level approximations. . . . . . . . . 14

2.3 The magnitude of the Green's function for the vector potential $G_{x x}^{4} 18$

2.4 The magnitude of the Green's function for the vector potential $G_{:=}^{A} \quad 18$

2.5 The magnitude of $\int G_{z x}^{A} d x \ldots \ldots \ldots$

2.6 The magnitude of the Green's function for the vector potential $G_{z r}^{\mathcal{A}} \quad 19$

2.i The magnitude of the Green's function for the scalar potential $G_{x}^{q_{e}} \quad 20$

2.S The magnitude of the Green's function for the scalar potential $G_{*}^{q_{\sigma}} \quad 20$

3.1 Strip of width $2 u$ located near interface between two semi-infinite half-spaces and illuminated by source in upper half-space . . . . 26

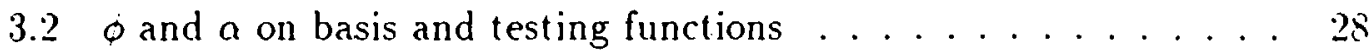

3.3 Single triangular testing and basis functions . . . . . . . . 29

3.4 Basis and testing functions on a single horizontal strip of width $2 \mathrm{~W} \quad 3.3$

4.1 Strip of width $2 u$ located near interface between two semi-infinite half-spaces and illuminated by source in upper half-space . . . . 43 
4.2 Normalized current density on a four wavelength strip for the TM excitation (reproduced from [15]). See Fig. 4.1 and the following text for the geometry and its parameters. . . . . . . . . . 45

4.3 Normalized current density on a four-wavelength strip from the spatial-domain MoM for the TM excitation. See Fig. 4.1 and the following text for the geometry and its parameters. . . . . . . . 45

4.4 Normalized current density on a strip below interface for $\theta=0^{\circ}$ and the TM excitation (reproduced from [16]). See Fig. 4.1 and the following text for the geometry and its parameters. . . . . . 46

4.5 Normalized current density on a strip below interface for $\theta=0^{\circ}$ and the TM excitation from the application of the spatial-domain MoM. See Fig. 4.1 and the following text for the geometry and its parameters.

4.6 Normalized current density on a strip below interface for $\theta=0^{\circ}$ and the TE excitation (reproduced from [17]). See Fig. t.1 and the following text for the geometry and its parameters. . . . . 47

$4 . \overline{7}$ Normalized current density on a strip below interface for $\theta=0^{\circ}$ and the TE excitation from the application of the spatial-domain MoM. See Fig. 4.1 and the following text for the geometry and its parameters. ...................... . . 4

4.8 A plane wave incident upon a conducting cylinder in a homogeneous medium . . . . . . . . . . . . . . . . . 4

4.9 The magnitude of the current density on a cylinder in a homogeneous medium for T.M excitation 
4.10 The magnitude of the current density on a cylinder in a homogeneous medium for TE excitation

4.11 Conducting cylinder located near interface between two semi-infinite half-spaces and illuminated by a plane wave in upper half-space. .

4.12 Normalized current density on a circular cylinder of radius $R=$ $0.375 \lambda_{a}$ for the TM excitation with various sampling points along the circumferential direction. See Fig. 4.11 and the following text for the geometry and its parameters. . . . . . . . . . . 54

4.13 Rectangular cylinder located near interface between two semi-infinite half-spaces and illuminated by a plane wave in upper half-space .

4.14 Normalized current density on a rectangular cylinder of cross section $0.25 \lambda_{a}$ (width) and $0.1 \lambda_{a}$ (height) for the TM excitation with various sampling points along the periphery. See Fig. 4.13 and the following text for the geometry and its parameters.

4.15 Normalized current density on a rectangular cylinder of cross section $0.25 \lambda_{a}$ (width) and $0.1 \lambda_{a}$ (height) for the TE excitation with various sampling points along the periphery. See Fig. 4.13 and the following text for the geometry and its parameters.

1.16 The real and imaginary parts of the nornalized current deusities for the TE excitation and for $\theta=0^{\circ}$

4.17 The real and imaginary parts of the normalized current densities for the TE excitation and for $\theta=-45^{\circ} \ldots \ldots \ldots$

4.18 The real and imaginary parts of the normalized current densities for the TM excitation and for $\theta=0^{\circ}$ 
4.19 The real and imaginary parts of the normalized current densities for the TM excitation and for $\theta=-45^{\circ}$

4.20 Normalized current densities on the strip for the TE excitation and $h=-0.1 \lambda_{b}$

4.21 Normalized current densities on the strip for the T.M excitation

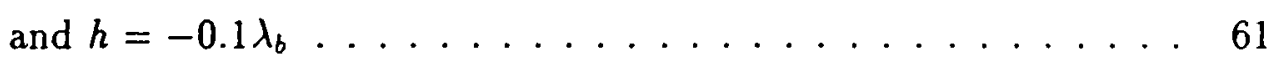

4.22 A two-strip, three layer geometry. . . . . . . . . . . . 62

4.23 The magnitudes of the current densities on the two strips for the TM excitation

4.24 The magnitudes of the current densities on the two strips for the

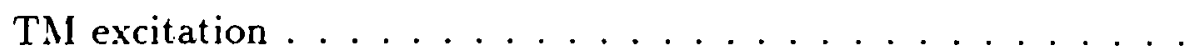

4.25 A geometry of ten strips located side by side in a homogeneous medium. . . . . . . . . . . . . . . . . .

4.26 The magnitude and phase of the current densities for the TE excitation, and for $k w=1.0 \ldots \ldots \ldots 66$

4.27 The magnitude and phase of the current densities for the TE excitation. and for $k w=2.0 \ldots \ldots \ldots 6$

4.28 The magnitude and phase of the current densities for the TE excitation, and for $k w=3.0 \ldots \ldots \ldots$

4.29 The magnitude and phase of the current densities for the TE excitation, and for $k w=4.0 \ldots \ldots$. . . . . . . . 6 i

4.30 The magnitude and phase of the current densities for the TE excitation. and for $k w=5.0 \ldots \ldots \ldots . \ldots . \ldots . \ldots$

1.31 The magnitude and phase of the current densities for the TM excitation, and for $k w=1.0 \ldots \ldots \ldots$. $\ldots \ldots \ldots$ 
4.32 The magnitude and phase of the current densities for the TM excitation, and for $k w=2.0 \ldots \ldots \ldots$

4.33 The magnitude and phase of the current densities for the TM excitation, and for $k w=3.0 \ldots \ldots \ldots 69 \ldots \ldots \ldots$

4.34 The magnitude and phase of the current densities for the TM excitation, and for $k w=4.0 \ldots \ldots \ldots$

4.35 The magnitude and phase of the current densities for the TM excitation, and for $k w=5.0 \ldots \ldots \ldots \ldots$

4.36 Three strips located in the same medium . . . . . . . . . 71

4.37 The magnitudes of the current densities on the three strips for the

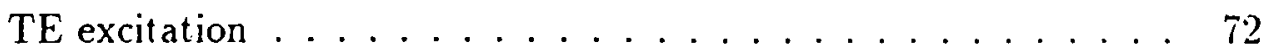

4.38 The magnitudes of the current densities on the three strips for the

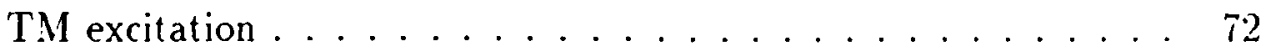

5.1 Strip of width $2 u$ located near interface between two semi-infinite half-spaces and illuminated by source in upper half-space . . . . i

5.2 The current magnitude of the iterative method for the T.M excitation when iteration number is increasing for $N=1000$

5.3 The current magnitude of the it erative method and LI' decomposition for the TM excitation and for $N=1000$.

5.4 The current magnitude of the iterative method for the TE excitation when iteration number is increasing for $N=1000$

5.5 The current magnitude of the iterative method and $\mathrm{Ll}^{\circ}$ decomposition for the TE excitation and for $N=1000 \ldots \ldots . \ldots 5$

5.6 The error plot of the iterative method for the T.II excitation and

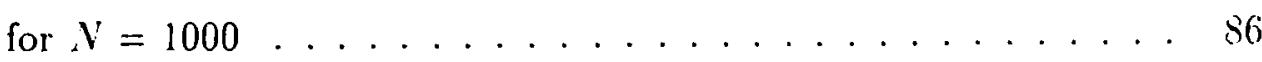


5.T The error plot of the iterative method for the TE excitation and for $N=1000 \ldots \ldots \ldots \ldots \ldots \ldots$

5.8 Two strips are located parallel to each other where the distance between them is $\lambda_{2} \ldots \ldots \ldots \ldots$

5.9 The magnitude of the current densities for the iterative method and $L C$ decomposition for the TM excitation, $N=1000$ and example $2 \ldots \ldots \ldots \ldots \ldots \ldots$

5.10 The magnitude of the current densities for the iterative method and $L C$ decomposition for the TE excitation, $N=1000$ and exam-

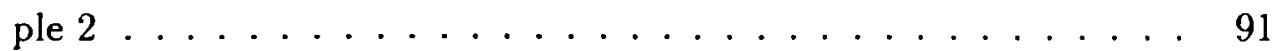

5.11 Three strips of width $2 w$ located in homogeneous medium with $0.5 w$ spacing between them on the same $z \ldots \ldots . \ldots 2$

5.12 The current magnitude of the iterative method and LU decomposition for $\theta=0^{\circ}$ and T.M excitation ............ . 94

5.13 The current magnitude of the iterative method and LU decomposition for $\theta=60^{\circ}$ and TM excitation ...........94

5.14 The current magnitude of the iterative method and $L U^{\top}$ decomposition for $\theta=0^{\circ}$ and TE excitation $\ldots \ldots \ldots . \ldots 95$

5.15 The current magnitude of the iterative method and LU! decomposition for $\theta=60^{\circ}$ and $\mathrm{TE}$ excitation 


\section{List of Tables}

4.1 The CPU times of the spectral domain, the spatial domain and the FFT approaches for TE and TM excitations

4.2 The CPU times of the spectral domain, the spatial domain and the FFT approaches for, (a) TE and (b) TM excitations

4.3 The C.PU times of the spectral domain, the spatial domain and the FFT approaches for the TE and TM excitations . . . . . . 64

5.1 The values of $\Lambda_{1}$ and $\Lambda_{2}$ for different $N$ for example $1 . \ldots$ is

5.2 (a) The CPL times of the iterative method and $\mathrm{LC}^{\circ}$ decomposition

(b) The number of iterations of the iterative method for the T.M excitation and example 1.

5.3 The CPU time and number of iterations of the iterative method for the TM excitation when $N$ is set to (a) 200. (b) 400 .

5.4 (a) The CPLI times of the iterative method and LI' decomposition (b) The number of iterations of the iterative method for the TE excitation and example 1.

5.5 The CPL time and number of iterations of the iterative method for the TE excitation when .1 is set to (a) 200 . (b) $100 \ldots . . .8$

5.6 The values of $\lambda_{1}$ and $l_{2}$ for various $\Lambda$ for example $2 \ldots \ldots$ 
5.7 (a) The CPU times of the iterative method and LL' decomposition (b) The iteration number of the iterative method for the TM excitation and example 2.

5.8 (a) The CPU times of the iterative method and LU! decomposition (b) The iteration number of the iterative method for the TE excitation and example 2.

5.9 The CPU times of the iterative method and LU decomposition for (a) TM and (b) TE excitations and (c) the iteration numbers of the TM and TE excitations when $\theta$ is $0^{\circ}$ and $60^{\circ} \ldots \ldots 9$ 


\section{Chapter 1}

\section{Introduction}

Adrances in high speed digital computers have led to the development of more sophisticated numerical methods to solve large electromagnetic problems of practical interest which, by classical techniques. would be virtually impossible. The basic techniques that are used in electromagnetic problems are mainly the method of moments (MoM) [1, 2]. finite element methods (FEM) [3] and the finite difference time domain (FDTD) methods [4], all of which basicly transform integral. differential or integro-differential equations into algebraic equations. Therefore. the computational efficiency of these techniques is dependent on the efficiency of forming a set of linear equations and the number of unknowns.

The Method of Moments is very popular for the solution of open field problems particularly for printed geometries in planar stratified media. It is presently. recognized as the most powerful approach for the analysis of printed antenna configurations and for the characterization of radiation and coupling phenomena in printed circuit discontinuities. A large number of applications of the $M 0.1 /$ can be found in the literature $[5,6,7]$. 
The main advantages of Mo.I are its accuracy. versatility and the ability to compute near -as well as far-zone parameters. The basic idea of the MoM is to reduce an integral equation to a matrix equation, and to solve the matrix equation by a known technique.

The first step of the Mo.M formulation is to write an integral equation describing the electromagnetic problem, which could be the mixed potential integral equation (MPIE) or the electric field integral equation (EFIE) for the printed geometries. These integral equations require related Green's functions. either of the vector and scalar potentials (for MPIE formulation) or of the electric fields (for EFIE formulation). Since the spectral-domain Green's functions are available in closed forms. their spatial-domain counterparts are obtained via an efficient inverse Fourier transform algorithm. Once the Green`s functions are obtained, the solution due to a general source in 2-D can be obtained by the principle of linear superposition. The next step in the Moll formulation is to expand the unknown function in terms of known basis functions with unknown coefficients. then is to implement the boundary condition in int egral sense through the testing procedure. Following these steps. the integral equation is transformed to a matrix equation, whose entries are double integrals in the spatial domain, one for the convolution integral to find the electric field, and one for the testing procedure to apply the boundary condition. However, in the spectral-domain application of the MoM. the matrix entries become single integrals over infinite domain. Consequently; the computational efficiency of the Moll lies in the evaluation of the MoM matrix entries. of course for moderate size geometries requiring a few hundred of unknowns. For a geometry requiring a large number of unknowns, the 
matrix solution time dominates the overall performance of the technique, therefore the efficiency of the method is defined by the efficiency of the linear system solver.

In this thesis, three different implementations of the MoM are studied, which are, namely, the spatial-domain MoM using the closed-form Green's functions. the spectral-domain MoM using the GPOF algorithm [10] to find the matrix entries, and the spectral-domain MoM using a FFT algorithm to evaluate the matrix entries. It is observed that the most efficient one is the spectral-domain MoM using the GPOF algorithm. However, for large geometries requiring a large number of unknowns, the efficiency of the overall method can be improved by using an iterative algorithm, developed in this thesis. for the solution of the matrix equation.

In Chapter 2, a method to obtain Green's functions in the spectral and spatial domains is presented. The details of the MoM formulations in the spectral and spatial domains are given in Chapter 3. Then. several numerical examples of these approaches are presented in Chapter 4. An efficient iterative method to solve the matrix equation is introduced in Chapter 5 with some examples. and finally: conclusions and fut ure work are given in Chapter 6. 


\section{Chapter 2}

\section{Green's Functions in Spectral and Spatial Domains}

Green's functions, either in the spatial or spectral domain. play an important role in driving integral equations for electromagnetic problems. Especially for planar multilayer geometries, they reduce the dimension of the problem from 3-D to 2.5D by incorporating the layer information. such as the dielectric constants. thicknesses and the number of layers. through satisfying the boundary conditions at the interfaces between the lavers. Therefore, the efficient calculation of Creen's functions is quite important for the efficiency of the method employed in the characterization of such geometries. In this chapter, the derivation of Creen's functions in the spectral domain is first presented, then their spatial domain counterparts are obtained in closed forms.

Green's functions of the vector and scalar potentials in the spectral and spatial domains are obtained for the sources of horizontal and vertical electric dipoles 
placed in multilayer planar media, where the layers are assumed to ext end to infinity in transverse directions. The spatial-domain Green's functions are obtained by taking the inverse Fourier transform of the corresponding Green ${ }^{\circ}$ functions in the spectral domain whose analytical expressions are available for planar. multilayer geometries. Hence, the main difficulty in obtaining the spatial-domain Green's functions is the transformation of the spectral-domain Green's functions. which can be done analytically for a few special cases. Here. we present a technique that will allow the spatial-domain Green's functions to be approximated in closed forms, and that will completely eliminate the computational difficulty.

To obtain the inverse Fourier transform analytically, Green`s functions in the spectral domain are approximated in terms of complex exponentials. One way to perform this approximation is to use Prony's method, in which the number of samples required must be twice the number of complex exponentials [ 8$]$. It is obvious that this leads to difficulty in sampling rapid variations of the spectral-domain Green's functions, unless a large number of exponentials is used. Although the least-square Prony method improves its ability to account for the rapid changes with a moderate number of exponentials [9], it still requires several trial and error iterations. because of its noise sensitivity, which makes the technique inefficient and not robust. Another exponential approximation technique is the generalized pencil of function (GPOF) method [10]. which is more robust and less noise sensitive when compared to the original and least-square Prony methods. and also provides a good measure for choosing the number of exponentials required in the approximation. However, it still requires a study of the spectral-domain beharjor of the Green's function in advance. in order to decide on the approximation parameters such as the number of sampling points and the maximum value of 
the sampling range. In addition, one would need to take thousands of samples in order to be able to approximate a slow converging function with rapid changes. because both the Prony and the GPOF methods require uniform sampling of the function.

Recently, a new approach based on a two-level approximation has been proposed to overcome the previously mentioned difficulties, and it has been demonstrated that the new approach is very robust and computationally much more efficient than the original one and its variants. The two-level approach divides the range of approximation into two parts, the first one covers the region where the function to be approximated has rapid transitions, whereas the function is smooth in the second region. Therefore, it is no longer necessary to take thousands of samples to account for a rapid transition that occurs in a small part of the entire range, resulting in a significant reduction in the number of data points to be processed. which, in turn, translates into a substantial saving in the computation time.

We will derive the spectral-domain Green's functions for planarly stratified media in Section (2.1). and will present the method of obtaining the closed-form spatial domain Green's functions in Section (2.2).

\subsection{Green's Functions in the Spectral Domain}

Consider the planar multilayered medium shown in Fig. 2.1 where it is assumed that the layers extend to infinity in the transverse directions. The source. (horizontal electric dipole (HED) or vertical electric dipole (VED)) is embedded in region $i$ and the observation point can be located in an arbitrary layer. Each 
layer can have different electric and magnetic properties $\left(\epsilon_{r_{1}}, \mu_{r_{1}}\right)$ and thickness $\left(d_{i}\right)$. Perfect electric conducting planes and half-spaces are also regarded as layers in this formulation.

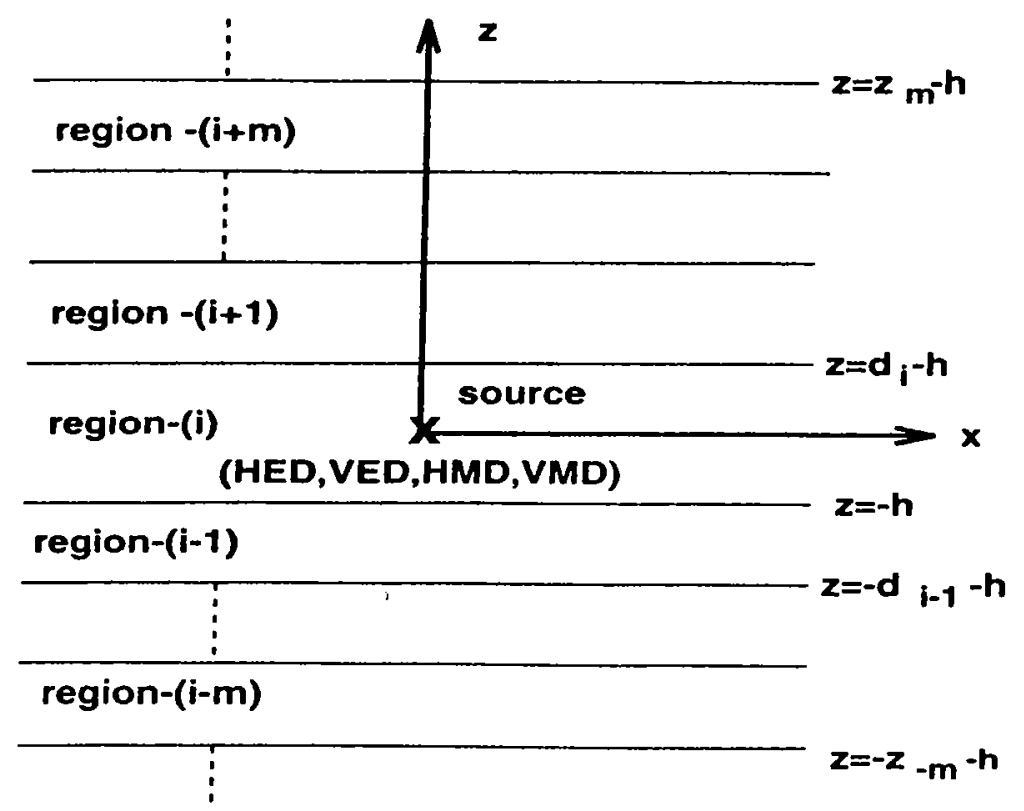

Figure 2.1: Sources embedded in a multilayer medium.

The spectral-domain Green`s functions are first derived in the source layer. then by using an iterative algorithm applied to each transverse electric (TE) and transverse magnetic (T.M) components they are obtained in the observation layer. Then, the spectral-domain Green`s functions are approximated in terms of complex exponentials via the GPOF method after the direct terms have been extracted.

All of the Green's functions, presented herein. are for the vector and scalar potentials that are indeed not defined uniquely in stratified media [11]. In other words. different sets of Green's functions for the vector and scalar potentials can be chosen to satisfy the same boundary conditions. The following notation for 
the Green's function is commonly used and referred to as the traditional form:

$$
\overline{\bar{G}}_{A}=(\hat{x} \hat{x}+\hat{y} \hat{y}) G_{x x}+\hat{z} \hat{x} G_{z x}+\hat{z} \hat{y} G_{z y}+\hat{z} \hat{z} G_{z z}
$$

for the vector potentials, $G_{x}^{q_{e}}$ and $G_{z}^{q_{e}}$ for the scalar potentials.

The spectral-domain Green's functions of the vector and scalar potentials can be obtained from the electric and magnetic fields generated by a current dipole $\mathbf{J}=I_{0} l \delta(\mathbf{r}) \hat{\alpha}$, where $\hat{\alpha}$ is a unit vector. For the sake of illustration, the field components for an HED in a multilayer media, Fig. 2.1, can be written in the source layer (layer $i$ ) in 2-D as follows [12]:

$$
\begin{aligned}
& \epsilon_{i} E_{z_{1}}=\frac{I_{0} l}{4 \pi \omega} \int_{-\infty}^{\infty} d k_{x} k_{x} e^{-j k_{x} x}\left[ \pm e^{-j k_{z_{1}}|z|}+B_{h}^{\epsilon} e^{j k_{z_{1}} z}+D_{h}^{\epsilon} \epsilon^{-j k_{z_{1}} z}\right] \\
& \mu_{i} H_{z_{i}}=-\mu_{i} \frac{I_{0} l}{4 \pi} \int_{-\infty}^{\infty} d k_{x} \frac{k_{x}}{k_{z_{1}}} \epsilon^{-j k_{x} x}\left[\epsilon^{-j k_{z_{1}}|z|}+A_{h}^{\epsilon} \epsilon^{j k_{z_{1}} z}+C_{h}^{\epsilon} \epsilon^{-j k_{z_{i}} z}\right]
\end{aligned}
$$

where the $z$ dependence of the fields in the source region is characterized as the sum of the direct term and up- and down-going waves due to the reflections from the boundaries at $z=-h$ and $z=d_{i}-h$. respectively, where + and signs are for positive and negative $z$ values, respectively: The coefficients of the up- and down-going waves can be obtained in terms of the generalized reflection coefficients by applying the appropriate boundary conditions: (i) the down-going waves for $z>0$ are the consequence of the reflections of the up-going waves at $z=d_{i}-h$ : (ii) the up-going waves for $z<0$ are the consequence of the reflections of the down-going waves at $z=-h$. It should be noted that the other field components can be easily derived from the z-components of the fields [12]. so they are not included here. After having obtained the field components. the components of the vector potential and the scalar potential can be derived from 
the following relations:

$$
\begin{array}{r}
\Gamma \times \mathbf{A}=\mu_{i} \mathbf{H} \\
o_{d}=-\frac{\Gamma \times \mathbf{A}}{j \omega \mu_{i} \epsilon_{i}}=\frac{I_{0} l}{j \omega} \frac{\partial \dot{\phi}}{\partial l^{\prime}}
\end{array}
$$

where $\phi_{d}$ and $\phi$ are the scalar potentials for the dipole element and a point charge, respectively, and $l^{\prime}$ is replaced by $x^{\prime}$ for an HED and replaced by $z^{\prime}$ for a VED. Below are the expressions of the spectral-domain Green's functions (traditional form) in the source layer for HED and VED sources. They read:

HED:

$$
\begin{aligned}
& \tilde{G}_{x x}^{A}=\frac{\mu_{i}}{2 j k_{z_{1}}}\left\{e^{-j k_{z_{1}}^{\prime}|z|}+A_{h}^{\epsilon} e^{j k_{z_{1}}=}+C_{h}^{\varepsilon} \epsilon^{-j k_{z_{1}} z}\right\} \\
& \dot{G}_{z x}^{A}=\frac{-\mu_{i}}{2 j k_{i=1}}\left\{\frac{k_{x} k_{z_{1}}}{k_{x}^{2}}\left(A_{h}^{\epsilon}+B_{h}^{\epsilon}\right) \epsilon^{j k_{z_{1}} z}+\frac{k_{x} k_{z_{1}}}{k_{x}^{2}}\left(D_{h}^{\epsilon}-C_{h}^{\epsilon}\right) \epsilon^{-j k_{z_{1}}=}\right\}
\end{aligned}
$$

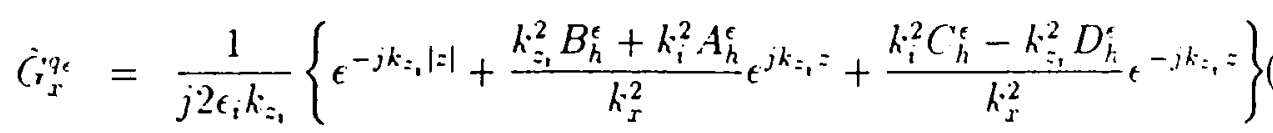

IED:

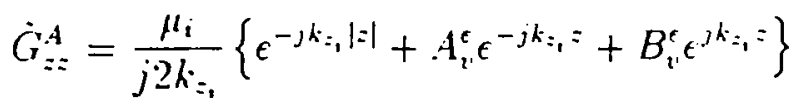

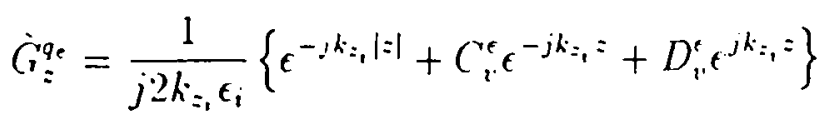

where $C_{i j}^{A}$ denotes the spectral-domain Green`s functions for the rector potential in the direction- $i$ due to a unit $j$-directed current element. $\left(i^{g e}\right.$ represents the Green s function of the scalar potential in the spectral domain due to a unit $i$-directed electric current element. $k_{1}^{2}=k_{x}^{2}+k_{z_{1}}^{2}$. the superscript $A$ represents 
the electric vector potential, and $q_{e}$ represents the electric scalar potential. The coefficients, $A_{h, v}^{e}, B_{h, v}^{e}, C_{h, v}^{e}$ and $D_{h, v^{*}}^{e}$ are functions of the generalized reflection coefficients $\tilde{R}_{T E, T M}$, and they are given by

$$
\begin{aligned}
& A_{h}^{e}=e^{-j k_{z_{1}}\left(d_{1}-h\right)} \tilde{R}_{T E}^{i, i+1}\left\{\epsilon^{-j k_{z_{1}}\left(d_{1}-h\right)}+\dot{R}_{T E}^{i, i-1} \epsilon^{-j k_{z_{1}}\left(d_{1}+h\right)}\right\} M_{i}^{T E} \\
& B_{h}^{\epsilon}=e^{-j k_{x_{1}}\left(d_{1}-h\right)} \tilde{R}_{T M}^{i, i+1}\left\{e^{-j k_{x_{1}}\left(d_{1}-h\right)}-\dot{R}_{T M}^{i, i-1} \epsilon^{-j k_{x_{1}}\left(d_{1}+h\right)}\right\} M_{i}^{T M} \\
& C_{h}^{e}=\epsilon^{-j k_{x_{1}} h} \tilde{R}_{T E}^{i, i-1}\left\{e^{-j k_{x_{i}} h}+\dot{R}_{T E}^{i, i+1} \epsilon^{-j k_{x_{1}}\left(2 d_{1}-h\right)}\right\} M_{i}^{T E} \\
& D_{h}^{e}=\epsilon^{-j k_{z_{1}} h} \tilde{R}_{T M}^{i, i-1}\left\{-\epsilon^{-j k_{z_{1}} h}+\dot{R}_{T M}^{i, i+1} e^{-j k_{z_{1}}\left(2 d_{1}-h\right)}\right\} M_{i}^{T M} \\
& A_{v}^{\epsilon}=\epsilon^{-j k_{z_{1}} h} \hat{R}_{T M}^{i, i-1}\left\{e^{-j k_{z_{1}} h}+\dot{R}_{T M}^{i, i+1} \epsilon^{-j k_{z_{1}}\left(2 d_{1}-h\right)}\right\} M_{i}^{T M} \\
& B_{v}^{\epsilon}=e^{-j k_{z_{1}}\left(d_{1}-h\right)} \tilde{R}_{T M}^{i, i+1}\left\{\epsilon^{-j k_{z_{1}}\left(d_{1}-h\right)}+\dot{R}_{T M}^{i, i-1} \epsilon^{-j k_{z_{1}}\left(d_{1}+h\right)}\right\} M_{i}^{T M H} \\
& C_{v}^{\epsilon}=\epsilon^{-j k_{z_{1}} h} \dot{R}_{T . M}^{i, i-1}\left\{-\epsilon^{-j k_{z_{1}} h}+\hat{R}_{T M}^{i, i+1} \epsilon^{-j k_{z_{2}}\left(2 d_{1}-h\right)}\right\} M_{i}^{T . M} \\
& D_{v}^{\varepsilon}=\epsilon^{-j k_{z_{1}}\left(d_{1}-h\right)} \dot{R}_{T M}^{i, i+1}\left\{-\epsilon^{-j k_{z_{1}}\left(d_{2}-h\right)}+\dot{R}_{T M}^{i, i-1} \epsilon^{-j k_{z_{1}}\left(d_{1}+h\right)}\right\} \cdot M_{i}^{T . M}
\end{aligned}
$$

where

$$
\begin{aligned}
& M_{i}^{T E . T M}=\left[1-\dot{R}_{T E . T M}^{i, i+1} \dot{R}_{T E, T M}^{i, i-1} \epsilon^{-j k_{z,} 2 d_{1}}\right]^{-1} \\
& \dot{R}_{T E . T . M}^{j+1 . j}=\frac{R_{T E . T . M}^{j+1 . j}+\dot{R}_{T E . T M M^{\mathrm{t}}}^{j, j-1}{ }^{-j k_{z}, 2 d,}}{1-R_{j, j+1} \dot{R}_{T E . T M}^{j . j-1} \mathrm{c}^{-j k_{z, j} 2 d_{j}}}
\end{aligned}
$$

Here $R$ and $\dot{R}$ are the Fresnel and the generalized reflection cocfficients [1:2] for which the subscripts $T E$ and $T . M$ represent the polarization of the wave. the 
superscripts $(i, i-1)$ or $(i . i+1)$ show the layer numbers, the subscripts $h$ and $v$ used in the coefficients $(2.11)-(2.18)$ represent the orientation of the source, horizontal and vertical, respectively, while the superscripts $\epsilon$ denotes the electric current source.

The amplitudes of the up- and down-going waves in a layer different from the source layer are related to those in the adjacent layers by,

$$
A_{j}^{-}=A_{j+1}^{-} \frac{T_{j+1, j} e^{-j\left(k_{z,+1}-k_{z},\right)\left(h+z_{-m+1}\right)}}{1-R_{j, j+1} \tilde{R}_{j, j-1} e^{-j k_{z}, 2 d_{j}}}
$$

where $A_{j}^{-}$and $A_{j+1}^{-}$are the amplitudes of the down-going waves in layers $j$ and $j+1$, respectively, $(j=i-m), T$ is the transmission coefficient, and $z_{-m}$ is the distance between the lower boundary of the source layer $i$ and the lower boundary of layer $j$. Fig. 2.1. Similarly the amplitudes of the up-going waves in layer $j=i+m$ can be written as

$$
A_{j}^{+}=A_{j-1}^{+} \frac{T_{j-1, j} \epsilon^{-j\left(k_{z_{j-1}}-k_{2,}\right)\left(z_{m-1}+d_{i}-h\right)}}{1-R_{j, j-1} \dot{R}_{j, j+1} \epsilon^{-j k_{z,} 2 d,}}
$$

Therefore, starting from the source layer, the field expressions for any layer can be obtained iteratively:

\subsection{Closed-Form Green's Functions in the Spa- tial Domain}

The scalar wave equation of a line source is written as

$$
\left[\frac{\partial^{2}}{\partial x^{2}}+\frac{\partial^{2}}{\partial z^{2}}+k_{\rho}^{2}\right] \phi(x . z)=-\delta(x) \delta(z)
$$


Because of the cylindrical symmetry of the problem, Eq. (2.23) is solved in cylindrical coordinates, i.e.,

$$
\left[\frac{\partial^{2}}{\partial \rho^{2}}+\frac{1}{\rho} \frac{\partial}{\partial \rho}+k_{\rho}^{2}\right] \phi(\rho)=-\delta(\rho)
$$

where $\delta(\rho)=\delta(x) \delta(z)$. It is known that, the solution of Eq. (2.24) is given as

$$
o(\rho)=\frac{-j}{4} H_{0}^{(2)}\left(k_{\rho} \rho\right)
$$

In addition, Eq. (2.23) can be solved by Fourier transform technique. For a fixed $z$, assuming that the Fourier transform of $\phi(x, z)$ exists, then it is expressible as an inverse Fourier transform integral as

$$
\phi(x, z)=\frac{1}{2 \pi} \int_{-\infty}^{\infty} \dot{\phi}\left(k_{x}, z\right) e^{-j k_{x} x} d k_{x}
$$

Consequently, on substituting (2.26) into (2.23), and using the fact that

$$
\delta(x)=\frac{1}{2 \pi} \int_{-\infty}^{\infty} e^{-j k_{x} x} d k_{x}
$$

one obtains

$$
\frac{1}{2 \pi} \int_{-\infty}^{\infty}\left[\frac{\partial^{2}}{\partial z^{2}}+k_{\rho}^{2}-k_{x}^{2}\right] \dot{\phi}\left(k_{x}, z\right) \epsilon^{-j k_{x} x} d k_{x}=-\frac{1}{2 \pi} \int_{-\infty}^{\infty} \delta(z) \epsilon^{-j k_{x} x} d k_{x}
$$

Therefore. we must have

$$
\left(\frac{\partial^{2}}{\partial z^{2}}+k_{z}^{2}\right) \dot{\phi}\left(k_{x}, z\right)=-\delta(z)
$$

where $h_{z}^{2}=k_{p}^{2}-k_{x}^{2}$. Thus.

$$
\dot{o}\left(k_{x}, z\right)=-\frac{j \epsilon^{-j k_{z} \mid=1}}{2 k_{z}}
$$

if only the outgoing-wave solution is considered. Hence. Eq. (2.26) becomes

$$
o(x . z)=\frac{-j}{4 \pi} \int_{-\infty}^{\infty} \frac{\epsilon^{-j k_{x} x-j k: \mid=1}}{k} d k_{x}
$$


By the uniqueness of the solution to the partial differential equation (2.23). Eq. (2.31) must be equal to Eq. (2.25) since both of them satisfy (2.23). As a result, an integral identity

$$
H_{0}^{(2)}\left(k_{\rho} \rho\right)=\frac{1}{\pi} \int_{-\infty}^{\infty} \frac{e^{-j k_{x} x-j k_{z}|z|}}{k_{z}} d k_{x}
$$

is obtained, which is used throughout this thesis quite frequently. The righthand side of Eq. (2.32) could be interpreted as an integral summation of the plane waves propagating in different directions including evanescent waves [12].

Since the principal goal of this section is to introduce a robust and efficient technique to obtain the spatial-domain Green's functions in closed-forms for planar layered media, it would be useful to first provide the definition of the spatialdomain Green's functions

$$
G^{A . q_{e}}=\frac{1}{2 \pi} \int_{-\infty}^{\infty} d k_{x} \epsilon^{-j k_{x} x} \tilde{G}^{A . q_{e}}
$$

where, $G$ and $\dot{G}$ are the Green's functions in the spatial and spectral domains. respectively, and SIP is the Sommerfeld integration path defined in Fig. 2.2.

The integral given in (2.33) cannot be integrated analytically, except for a fell special cases. However, if the spectral-domain representation of the Green's function. $\dot{G}$ in the integrand can be approximated in terms of complex exponentials, the analytical evaluation of the integral (2.33) becomes possible and can be expressed in terms of summation of the Hankel functions of the second kind. Therefore. the crucial step in the derivation of the closed-form Green s functions is the exponential approximation of $\dot{G}$.

The original approach of getting the closed-form Green's functions in the spatial domain has had some difficulties for example, the quasi-dynamic images and the surface wave poles need to be found and extracted from the Giren's 


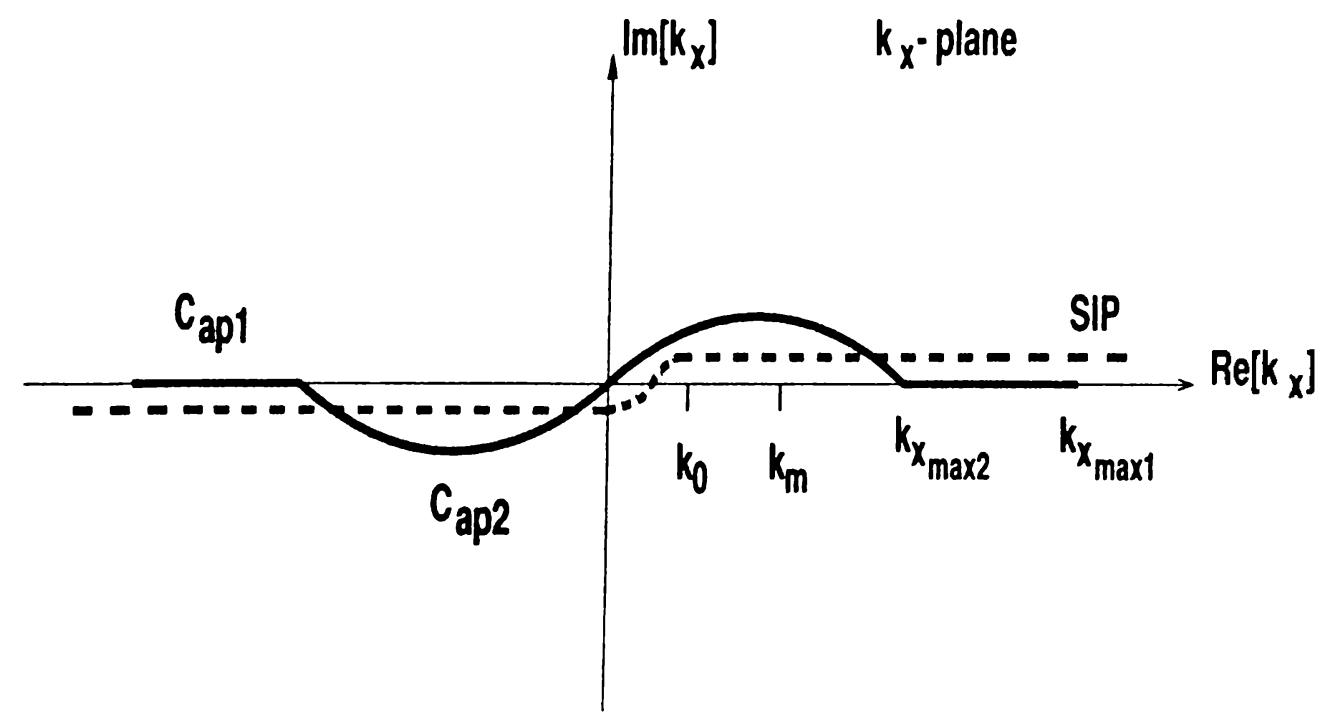

Figure 2.2: Definition of the Sommerfeld integration path, and the paths $C_{a p 1}$ and $C_{\text {ap } 2}$ used in two-level approximations.

function prior to the approximation. However, with the introduction of the twolevel approach. which is robust and very efficient, these difficult ies are eliminated [13]. In the two-level approach, a path formed by the paths $C_{a p 2}$ and $C_{a p l}$ is employed, as depicted in Fig. (2.2). and the paths $C_{a p 1}$ and $C_{a p 2}$ are defined by the following parametric equations:

$$
\begin{array}{ll}
\text { For } C_{a p 1} & k_{i_{1}}=-j k_{i}\left[T_{02}+t\right] \quad 0 \leq t \leq T_{01} \\
\text { For } C_{a p 2} & k_{i_{1}}=k_{i}\left[-j t+\left(1-\frac{t}{T_{02}}\right)\right] \quad 0 \leq t \leq T_{02}
\end{array}
$$

where $t$ is the running variable sampled uniformly in the corresponding range. The exponential approximation process begins with sampling the function to be approximated, and then the algorithm for exponential approximation is employed for the sampled values of the function. After having sampled the spectral-domain Green's function to be approximated, apart from the term $1 / j 2 k$, . the GPOF method is used to obtain the exponential approximation of the function. Which 
results in an approximation as follows:

$$
\begin{aligned}
\tilde{G}= & \frac{1}{j 2 k_{z_{1}}}\left\{\epsilon^{-j k_{z_{1}}\left|z-z^{\prime}\right|}+\sum_{n_{1}=1}^{N_{1}} C_{n_{1}} \epsilon^{-a_{n_{1}} k_{z_{1}}} \epsilon^{j k_{z_{1}}\left(z+z^{\prime}\right)}\right. \\
& +\sum_{n_{2}=1}^{N_{2}} C_{n_{2}} \epsilon^{-a_{n_{2}} k_{z_{1}}} e^{j k_{z_{1}}\left(z-z^{\prime}\right)}+\sum_{n_{3}=1}^{N_{3}} C_{n_{3}} \epsilon^{-a_{n_{3}} k_{z_{1}}} \epsilon^{-j k_{z_{1}}\left(z+z^{\prime}\right)} \\
& \left.+\sum_{n_{2}=1}^{N_{2}} C_{n_{2}} \epsilon^{-a_{n_{2}} k_{z_{1}}} e^{-j k_{z_{1}}\left(z-z^{\prime}\right)}\right\}
\end{aligned}
$$

where $C_{n}$ 's and $\alpha_{n}$ 's denote the coefficients and exponents of the complex exponentials obtained via the GPOF method.

To demonstrate the procedure on a real example. the derivation of the closedform Green's function in the spatial domain $G_{x x}^{A}$ is given below: first, the spectral domain representation is written

$$
\begin{aligned}
& \dot{G}_{x X}^{A}=\frac{1}{j 2 k_{z_{1}}}\left(\epsilon^{-j k_{1}\left|:-z^{\prime}\right|}+\tilde{R}_{T E}^{i, i+1} M_{i}^{T E} \epsilon^{-j k_{z_{1}} 2 d_{1}} \epsilon^{j k_{z_{1}}\left(=+z^{\prime}\right)}\right. \\
& +\dot{R}_{T E}^{i, i-1} \hat{R}_{T E}^{i, i+1} \cdot M_{i}^{T E^{-j k_{z}} 2 d_{1}} \epsilon^{j k_{z_{1}}\left(z-z^{\prime}\right)}+\dot{R}_{T E}^{i, i-1} \cdot M_{i}^{T E_{\epsilon}-j k_{z_{1}}\left(z+z^{\prime}\right)}
\end{aligned}
$$

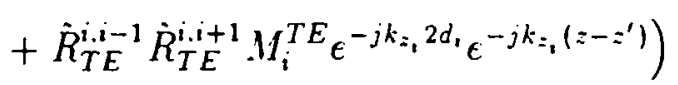

then its approximation by complex exponentials is transformed into the spatial domain as

$$
\begin{aligned}
& G_{x x}^{A}=\frac{1}{2 \pi} \int_{-\infty}^{x} d k_{x} \epsilon^{-j k_{x} x} \tilde{G}_{x x}^{A}
\end{aligned}
$$

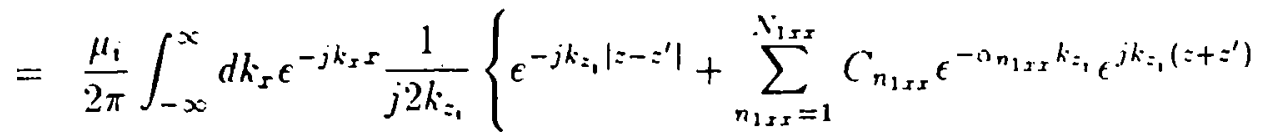

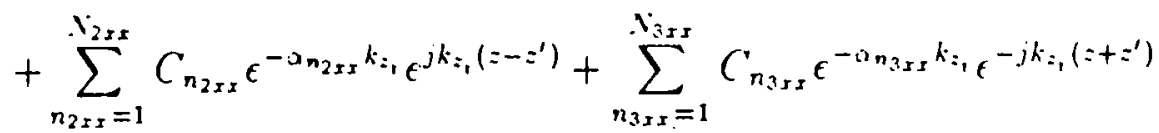

$$
\begin{aligned}
& \left.+\sum_{n_{2 x x}=1}^{r_{2 x x}} C_{n_{2 x x}} \epsilon^{-a_{n 2 x} k_{i,}} \epsilon^{-j k_{i 1}\left(z^{\prime}-z^{\prime}\right)}\right\}
\end{aligned}
$$

where $C_{n_{1 x}}$ and $\alpha_{n_{1 x}}(i=1,2,3)$ are the coefficients and exponents of the complex exponentials approximating the three term in Eq. (2.37). l'sing the identity 
derived in Eq. (2.32), the spatial-domain Green's function $G_{r x}^{A}$ is obtained as

$$
\begin{aligned}
G_{x x}^{A}= & -\frac{j \mu_{i}}{4}\left\{H_{0}^{(2)}\left(k_{i} \rho\right)+\sum_{n_{1 x x}=1}^{N_{1 x x}} C_{n_{1 x x}} H_{0}^{(2)}\left(k_{i} \rho_{n_{1 x x}}\right)+\sum_{n_{2 x x}=1}^{N_{2 x x}} C_{n_{2 x x}} H_{0}^{(2)}\left(k_{i} \rho_{n_{2 x x}}^{(1)}\right)\right. \\
& \left.+\sum_{n_{3 x x}=1}^{N_{3 x x}} C_{n_{3 x x}} H_{0}^{(2)}\left(k_{i} \rho_{n_{3 x x}}\right)+\sum_{n_{2 x x}=1}^{N_{2 x x}} C_{n_{2 x x}} H_{0}^{(2)}\left(k_{i} \rho_{n_{2 x x}}^{(2)}\right)\right\}
\end{aligned}
$$

where

$$
\begin{aligned}
\rho & =\sqrt{\left(x-x^{\prime}\right)^{2}+\left(z-z^{\prime}\right)^{2}} \\
\rho_{n_{1 x x}} & =\sqrt{\left(x-x^{\prime}\right)^{2}+\left(z+z^{\prime}+j a_{n_{1 x x}}\right)^{2}} \\
\rho_{n_{2 x x}}^{(1)} & =\sqrt{\left(x-x^{\prime}\right)^{2}+\left(z-z^{\prime}+j a_{\left.n_{2 x x}\right)^{2}}\right.} \\
\rho_{n_{2 x x}}^{(2)} & =\sqrt{\left(x-x^{\prime}\right)^{2}+\left(z-z^{\prime}-j a_{\left.n_{2 x x}\right)^{2}}\right.} \\
\rho_{n_{3 x x}} & =\sqrt{\left(x-x^{\prime}\right)^{2}+\left(z+z^{\prime}-j \alpha_{\left.n_{3 x x}\right)^{2}}\right.}
\end{aligned}
$$

It is known that $G_{y y}^{A}$ is equal to $G_{x x}^{A}$. and the formulation procedure for $C_{x}^{q_{e}} . G_{z}^{q_{e}}$ and $G_{z z}^{A}$ are similar to $G_{x x}^{A}$. For the derivation of $G_{z x}^{A}$, one should note that $k_{x}$ in the numerator of its spectral domain representation (2.7) should be eliminated in the approximation process. Therefore, $-j k_{s}$ is taken out from the expression. and $i_{z x}^{A} /\left(-j k_{s}\right)$ is approximated:

$$
\begin{aligned}
& \frac{\dot{G}_{z_{r}}^{A}}{-j k_{r}}=\frac{\mu_{i}}{2 j k_{z,}}\left\{\frac{k_{z=1}}{j k_{x}^{2}}\left(A_{h}^{\epsilon}+B_{h}^{\varepsilon}\right) \epsilon^{j k_{z_{1}}=}+\frac{k_{z_{1}}}{j k_{r}^{2}}\left(D_{h}^{e}-C_{h}^{e}\right) \epsilon^{-j k_{i_{1}}=}\right\}
\end{aligned}
$$

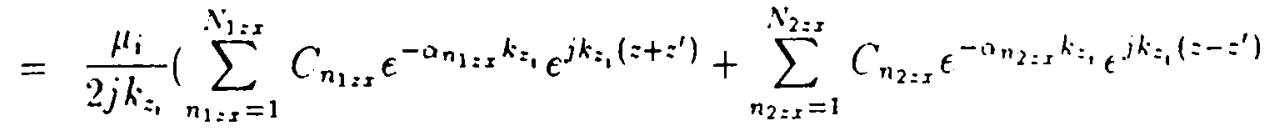

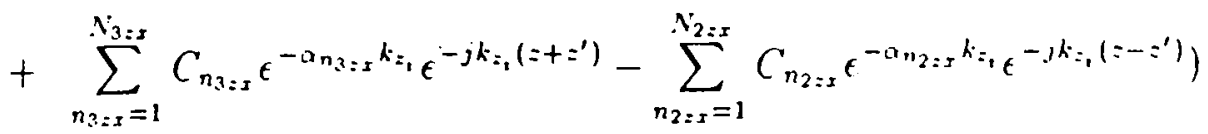

Vsing the following property of the Fourier transform.

$$
\begin{aligned}
& G(x, z) \longleftrightarrow \dot{C}\left(k_{x}, z\right) \\
& \int d x G(x, z) \longleftrightarrow \frac{\dot{G}\left(k_{s} . z\right)}{-j k_{r}}
\end{aligned}
$$


the integral of $G_{z x}^{A}$ is obtained as

$$
\begin{aligned}
\int d x G_{z x}^{A}= & \frac{j \mu_{i}}{4}\left\{\sum_{n_{1 x x}=1}^{N_{1 x x}} C_{n_{1 x x}} H_{0}^{(2)}\left(k_{i} \rho_{n_{1 x x}}\right)+\sum_{n_{2: x}=1}^{N_{2: x}} C_{n_{2: x}} H_{0}^{(2)}\left(k_{i} \rho_{n_{2: x}}^{(1)}\right)\right. \\
& \left.+\sum_{n_{3: x}=1}^{N_{3 x x}} C_{n_{3 x x}} H_{0}^{(2)}\left(k_{i} \rho_{n_{3 z x}}\right)-\sum_{n_{2 x x}=1}^{N_{2: x}} C_{n_{2: x}} H_{0}^{(2)}\left(k_{i} \rho_{n_{2: x}}^{(2)}\right)\right\}(2 .) 0
\end{aligned}
$$

Since the derivative of Hankel function is given by

$$
\frac{d\left(H_{0}^{(2)}(\alpha x)\right)}{d x}=-\alpha H_{1}^{(2)}(\alpha x)
$$

$G_{z x}^{A}$ can be obtained as

$$
\begin{aligned}
G_{z x}^{.4} & =-\frac{j \mu_{i}}{4}\left\{\sum_{n_{1 x x}=1}^{N_{1 x x}} C_{n_{1 x x}} \frac{x-x^{\prime}}{\rho_{n_{12 x}}} H_{1}^{(2)}\left(k_{i} \rho_{n_{12 x}}\right)+\sum_{n_{2: x}=1}^{N_{2 x x}} C_{n_{2: x}} \frac{x-x^{\prime}}{\rho_{n_{2 x x}}^{(1)}} H_{1}^{(2)}\left(k_{i} \rho_{n_{2: x}}^{(1)}\right)\right. \\
& \left.+\sum_{n_{3: x}=1}^{N_{z x} 3} C_{n_{3 z x}} \frac{x-x^{\prime}}{\rho_{n_{3: x}}} H_{1}^{(2)}\left(k_{i} \rho_{n_{3 x x}}\right)-\sum_{n_{2: x}=1}^{N_{2: x}} C_{n_{2: x}} \frac{x-x^{\prime}}{\rho_{n_{2}: x}^{(2)}} H_{1}^{(2)}\left(k_{i} \rho_{n_{2: x}}^{(2)}\right)\right\}(2.52)
\end{aligned}
$$

To give an idea how these closed-form Green's functions behave. an example is provided for two layer media: the first layer has a relative permittivity of 4 and the second laver is free space, the source is placed at the interface and hence $z=z^{\prime}=0$. The plot of Green's functions are given below. 


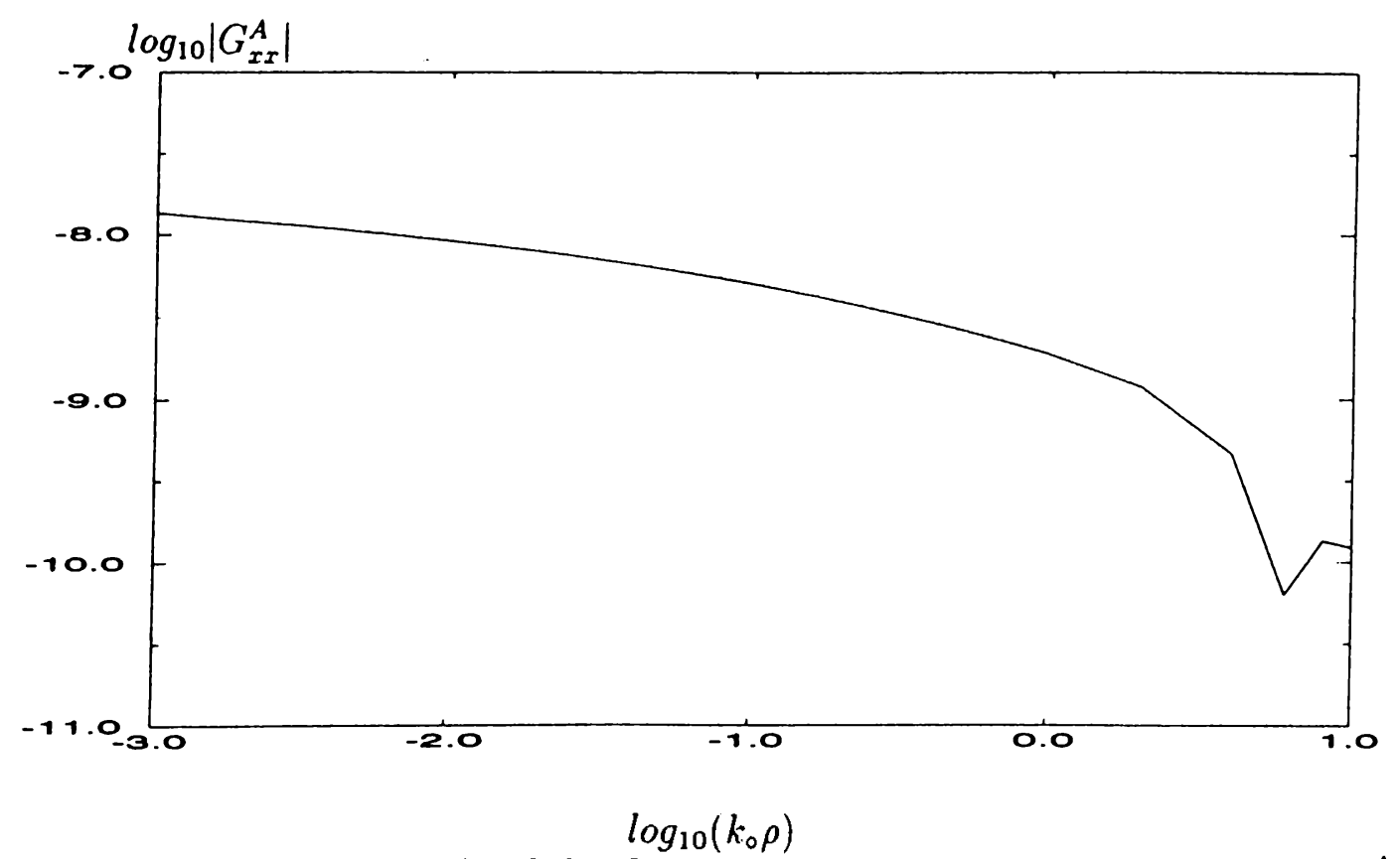

Figure 2.3: The magnitude of the Green's function for the vector potential $G_{x r}^{A}$

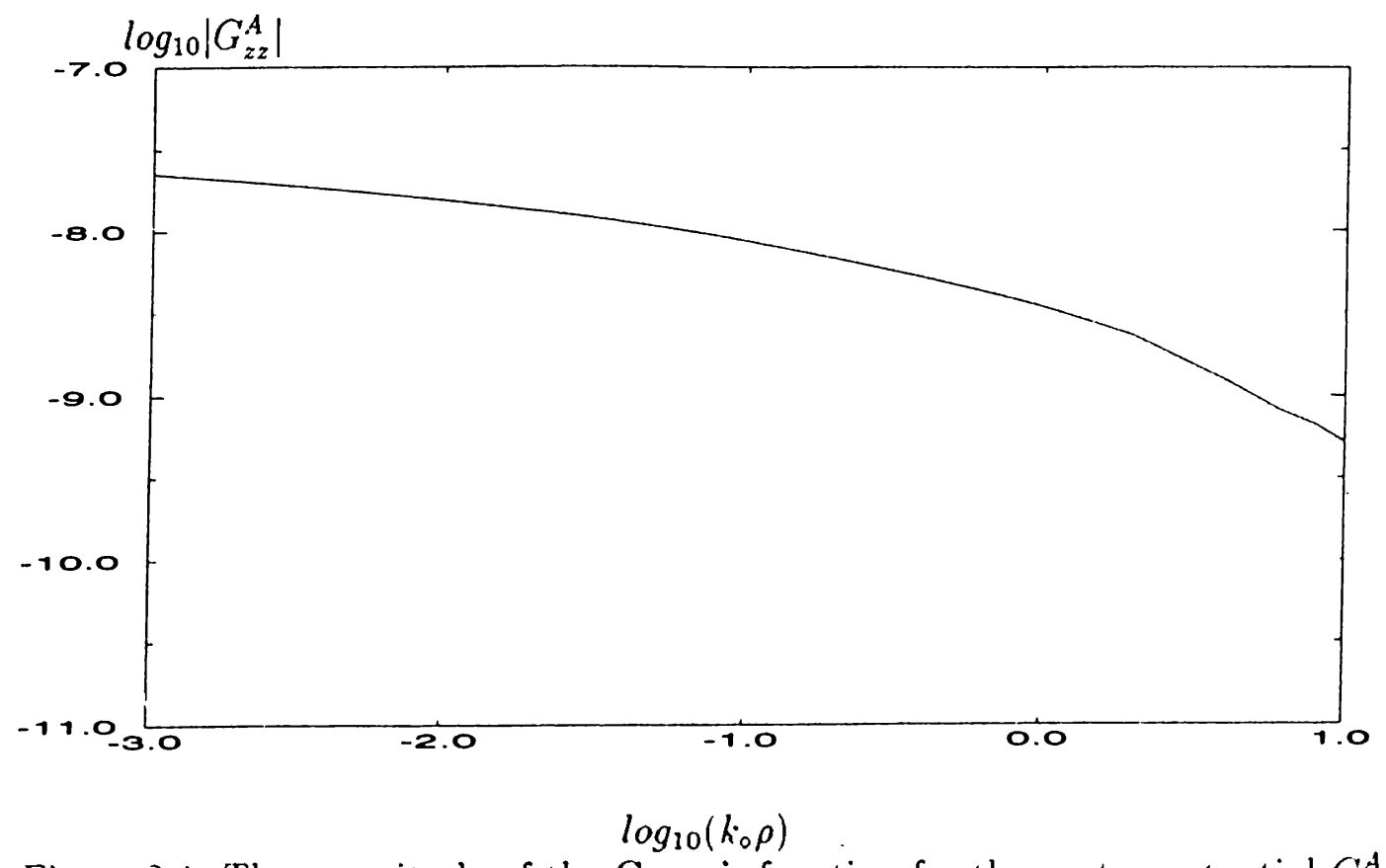

Figure 2.4: The magnitude of the Green's function for the vector potential $G_{z:}^{A}$ 


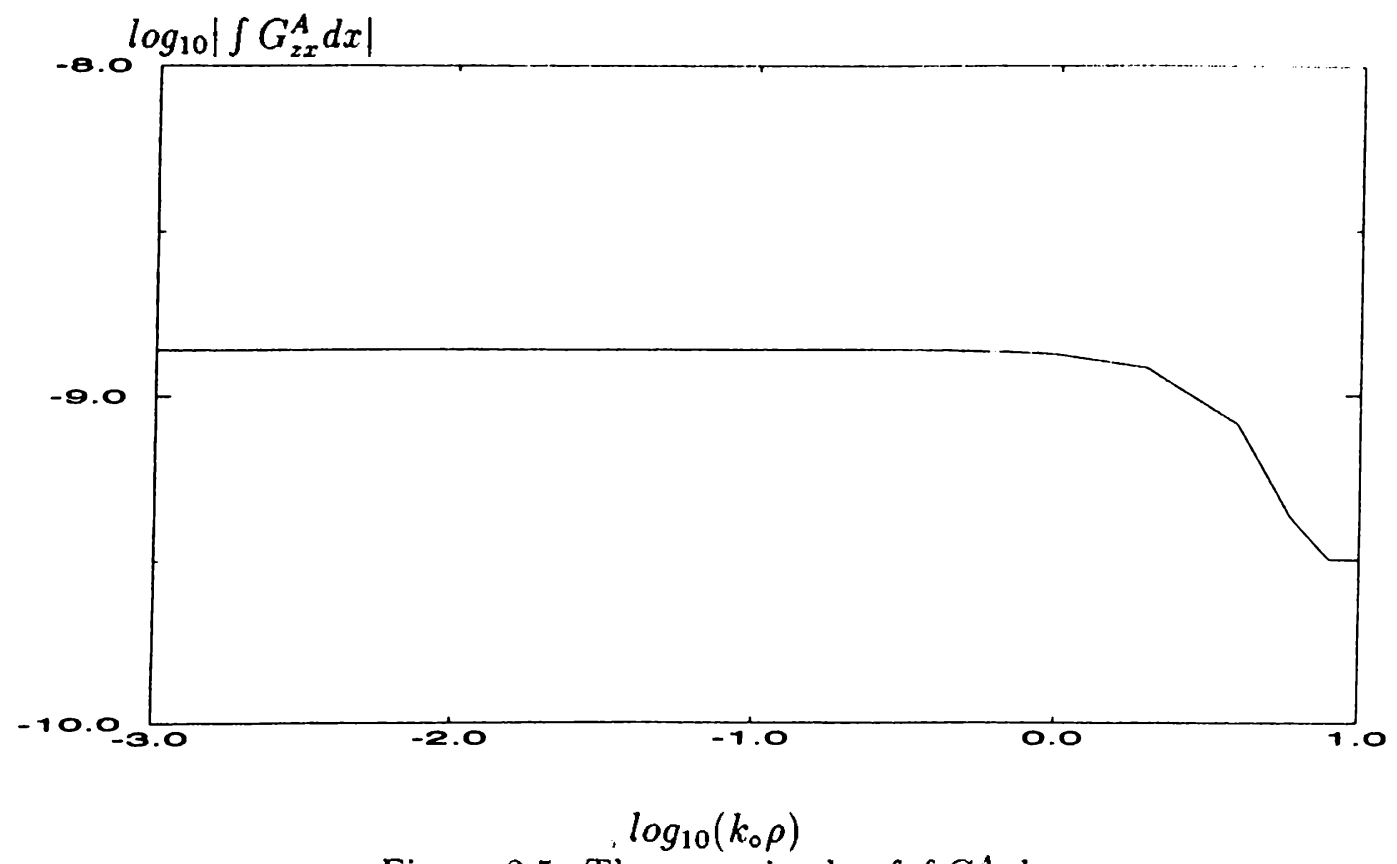

Figure 2.5: The magnitude of $\int G_{z x}^{A} d x$

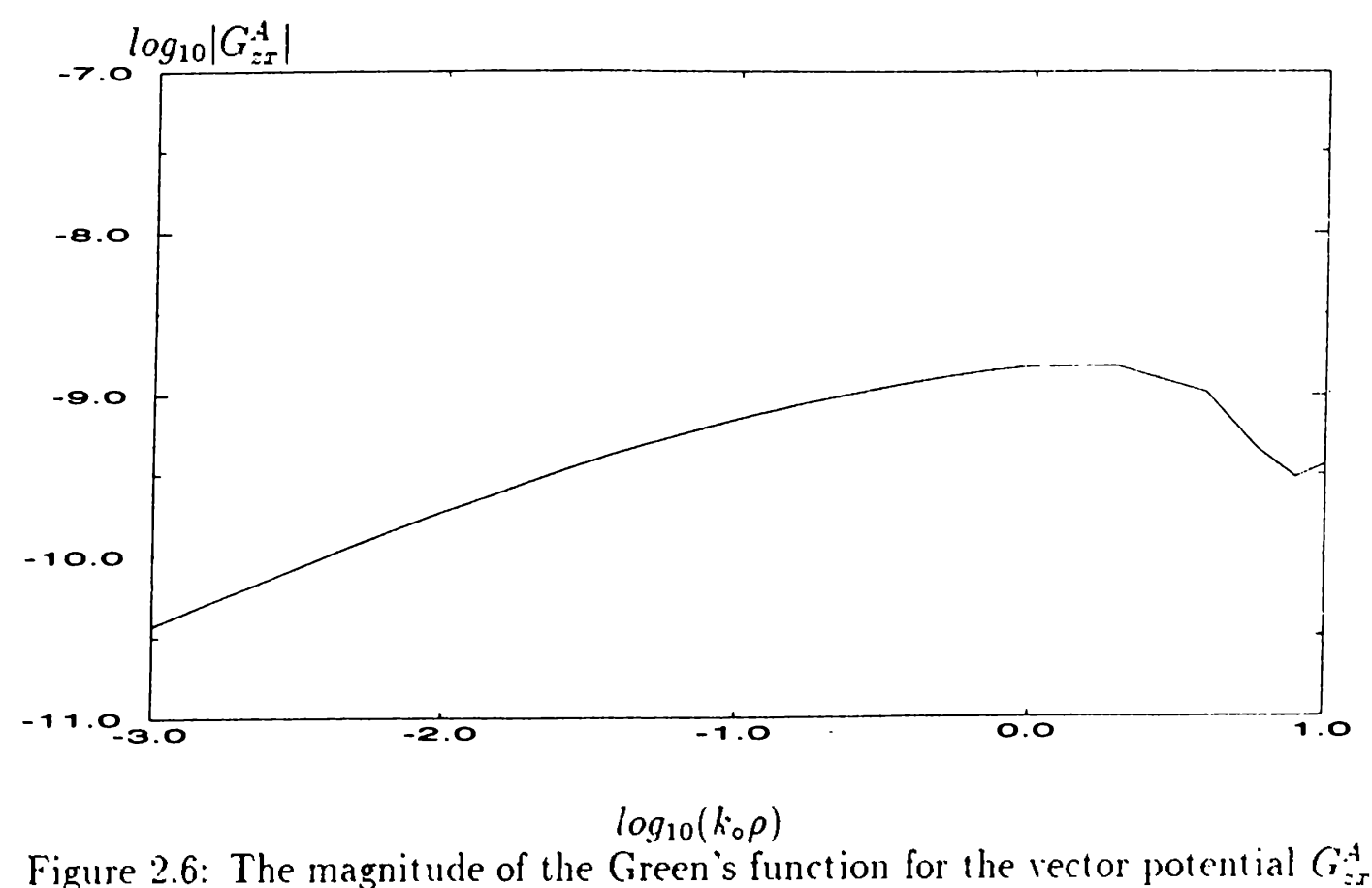




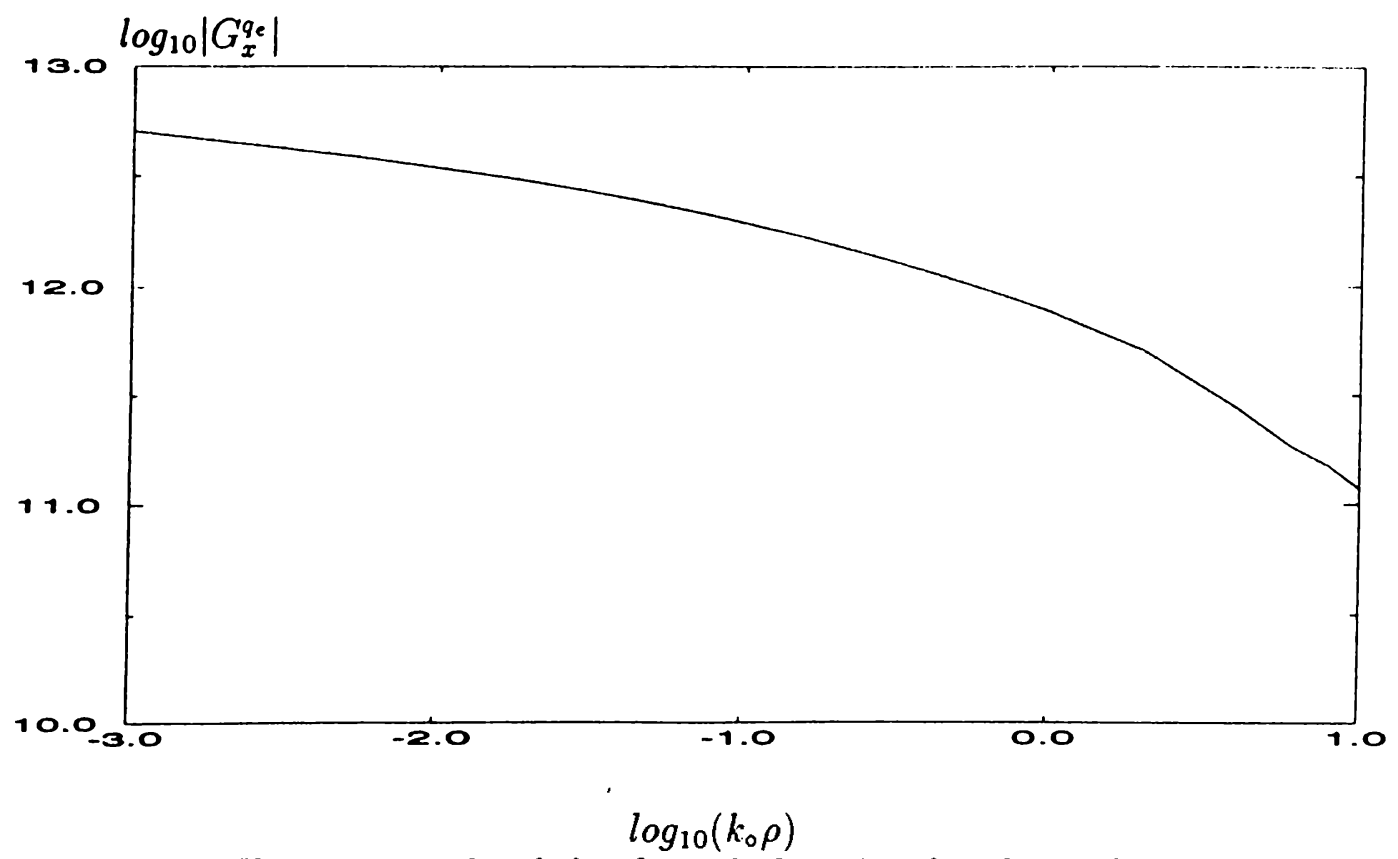

Figure 2.7: The magnitude of the Green's function for the scalar potential $G_{x}^{q e}$

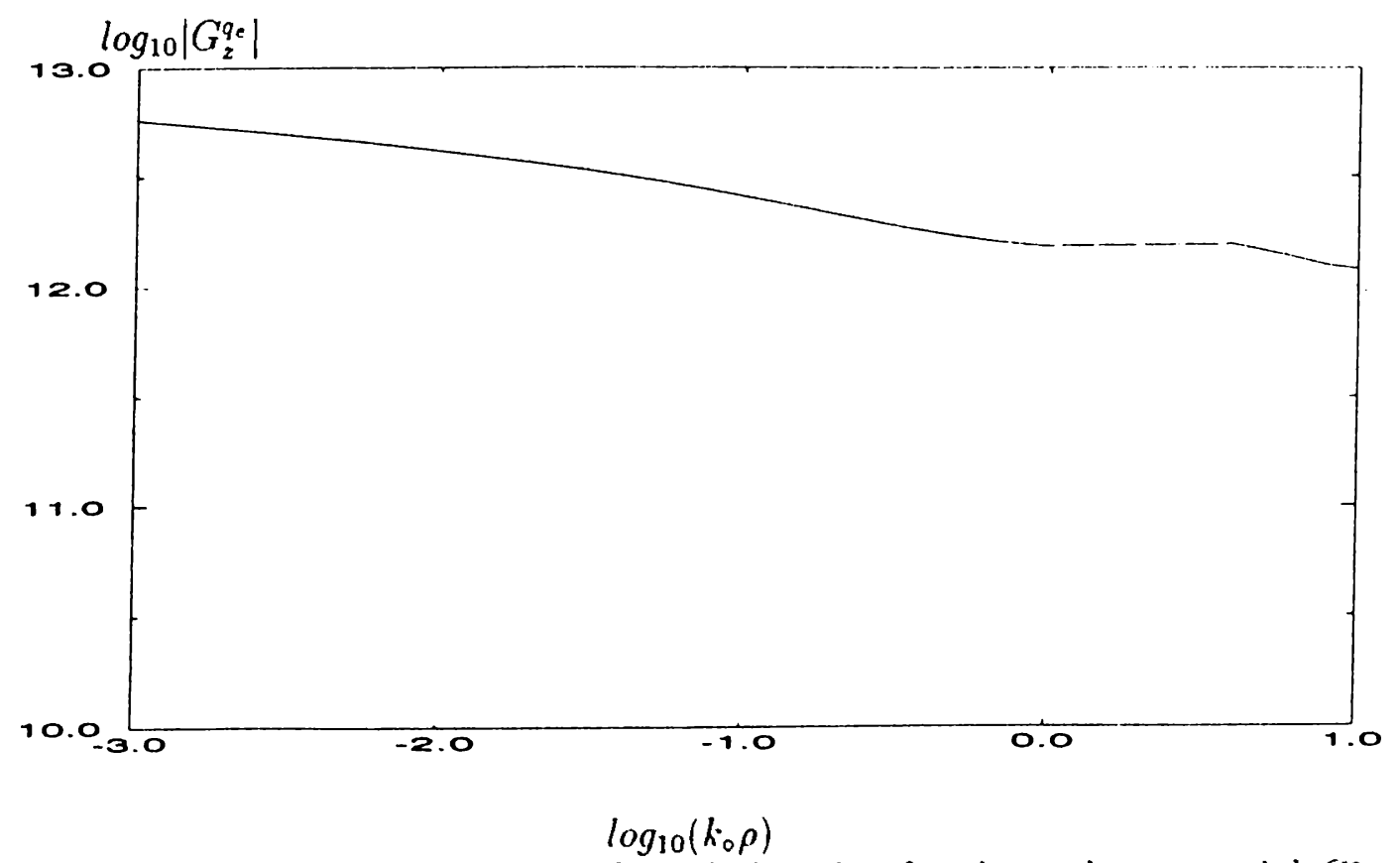

Figure 2.S: The magnitude of the Green`s function for the scalar potential Cige 


\section{Chapter 3}

\section{Formulation of Electric Field Integral Equation in 2-D}

Method of Moments is one of the most popular numerical techniques employed in electromagnetic simulations of printed circuits, and antennas. Hence, in this thesis we are mainly concerned with the analy'sis of 2-D printed structures with the use of the various forms of the MoM. Although the main algorithm is the same in these forms. which is the discretization of unknown in terms of known basis functions and application of the boundary condition: in integral sense. the implementation of these steps can be carried out in different domains or via different algorithm. For example, the whole algorithm can be carried out in the spatial and spectral domain, or the implementation of the boundary condition in integral sense can be performed via the fast. Fourier transform. 


\subsection{Method of Moments}

Although the $\mathrm{MlOM}$ in this thesis is applied for the solution of an integral equation. the general setting of the $\mathrm{MoM}$ is given here for the sake of completeness. For this purpose, the following operator equation is considered,

$$
L(f)=g
$$

where $g$ represents a known source contribution. $L$ denotes a linear operator operating on an unknown parameter $f$, that has to be determined. The first step in the application of the MoM for the solution of Eq. (3.1) is to expand the unknown parameter by a set of known basis functions with unknown coefficients as

$$
f=\sum_{n} a_{n} f_{n}
$$

where $f_{n}$ represents the basis function spanning the domain of the operator $L$. and $a_{n}$ is the corresponding unknown coefficient. Ising the representation of $f$ in Eq. (3.2) and the linearity property of $L$, the following equation is obtained:

$$
\sum_{n} a_{n} L\left(f_{n}\right)=g
$$

The second step in MoM is to use a set of weighting functions. or testing functions. $w_{1}, w_{2}, w_{3} \ldots$ in the range space of $L$. and to take their inner products with both sides of Eq. (3.3). resulting in

$$
\begin{aligned}
& \left\langle w_{1} . g\right\rangle=\sum_{n} a_{n}\left\langle w_{1} \cdot L \cdot f_{n}\right\rangle \\
& \left\langle u_{2} \cdot g\right\rangle=\sum_{n} a_{n}\left\langle w_{2}, L f_{n}\right\rangle
\end{aligned}
$$


These equations can be written in matrix form as

$$
\left[g_{m}\right]=\left[l_{m n}\right]\left[a_{n}\right]
$$

where

$$
\begin{gathered}
{\left[g_{m}\right]=\left[\begin{array}{c}
<w_{1} . g> \\
<w_{2} . g> \\
\vdots
\end{array}\right]} \\
{\left[l_{m n}\right]=\left[\begin{array}{c}
<w_{1}, L f_{1}><w_{1}, L f_{2}>\ldots \\
<w_{2}, L f_{1}><w_{2}, L f_{2}>\ldots \\
\ldots \ldots \ldots \ldots \ldots \ldots \ldots
\end{array}\right]} \\
{\left[\alpha_{n}\right]=\left[\begin{array}{c}
\alpha_{1} \\
\alpha_{2} \\
\vdots
\end{array}\right]}
\end{gathered}
$$

If the matrix $[l]$ is nonsingular. then

$$
\left[\alpha_{n}\right]=\left[l_{m n}\right]^{-1}\left[g_{m}\right]
$$

and the solution for $f$ is obtained from Eq. (3.2). If the matrix [l] is of infinite order, then its inversion can not be performed unless [I] is diagonal. If the sets $f_{n}$ and $w_{n}$ are finite. the matrix is of finite order. and can be inverted by known methods.

The solution depends on the choice of the basis functions. which should be linearly independent and should be chosen such that some linear combination Eq. (3.2) can approximate f reasonably well. The solution also depends on the set of testing function $w_{1}, w_{2} \ldots .$. which should be linearly independent and should best represent the properties of $g$. Some additional factors which atfect 
the choice of $f_{n}$ and $w_{n}$ are (1) the accuracy of solution desired. (2) the ease of evaluation of the matrix elements, (3) the size of the matrix that can be inverted. and (4) the realization of a well-conditioned matrix [l] [1].

The name method of moments derives from the original terminology that $\int x^{n} f(x) d x$ is the nth moment of $f$. When $x^{n}$ is replaced by an arbitrary $w_{n}$. we continue to call the integral a moment of $f$. The name method of weighted residuals derives from the following interpretation. If Eq. (3.3) represents an approximate equality, then the difference between the exact and approximate $L f$ is

$$
g-\sum_{n} a_{n} L f_{n}=r
$$

which is called the residual $r$. The inner products $\left\langle w_{n}, r\right\rangle$ are called the weighted residuals. Eq. (3.4) is obtained simply by setting all weighted residuals to zero.

\subsection{Electric Field in 2-D}

The general form of scattered electric fields can be written as

$$
\begin{aligned}
& E_{x}=-j u G_{r s}^{4} * J_{s}+\frac{1}{j u} \frac{\partial}{\partial r}\left(C_{r}^{q e} * \Gamma \cdot \mathbf{J}\right) \\
& E_{y}=-j u C_{y y}^{4} * J_{y}+\frac{1}{j u} \frac{\partial}{\partial y}\left(C^{q_{e}} * \Gamma \cdot \mathbf{J}\right) \\
& E_{z}=-j w G_{z=x}^{A} * J_{x}-j u C_{z y}^{A} * J_{y}-j u G_{z:}^{A} * J_{z}+\frac{1}{j w} \frac{\partial}{\partial z}\left(C^{* r} * \Gamma \cdot \mathbf{J}\right)
\end{aligned}
$$

where * denotes convolution. and $G_{x x}^{A}=G_{y y}^{4}$. The fields $E_{x}$. $E_{y}$ and $E_{\text {: represent }}$ the scattered electric fields in $x, y$ and $z$ directions. respectively. The term Gid represents $i$-directed vector potential at $\mathbf{r}$ due to a $j$-directed clectric dipole of unit strength located at $\mathbf{r}^{\prime}$. While $G^{q_{e}}$ represents the scalar potcmtial by a unit 
point charge associated with an electric dipole. Since the traditional form of the Cireen's functions is employed in the formulation, Gireen's function of the scalar potential is not unique for horizontal and vertical electric dipoles as stated previously. Hence, the term involving the Green's function of the scalar potential. which is common in Eqs. (3.9)-(3.11), can be explicitly written as

$$
G^{q_{e}} * \Gamma \cdot \mathbf{J}=C_{x}^{q_{e}} * \frac{\partial J_{x}}{\partial x}+G_{y}^{z_{e}} * \frac{\partial J_{y}}{\partial y}+C_{i}^{\prime *} * \frac{\partial J_{z}}{\partial z}
$$

where $C_{s}^{q_{e}}=C_{y}^{q_{e}}$ and $C_{z}^{q_{e}}$ denote Green's functions of the scalar potential for a horizontal and vertical electric dipoles, respectively. Howerer. the equations (3.9)-(3.11) can be simplified in $2-\mathrm{D}$, because $J_{x}, J_{y}$ and $J_{z}$ are functions of $x$ and $z$ only, that is,

$$
\begin{aligned}
\frac{\partial}{\partial y}\left(G^{q_{e}} * \Gamma \cdot \mathbf{J}\right) & =0 \\
\frac{\partial J_{y}}{\partial y} & =0
\end{aligned}
$$

which result in the following scattered electric field equations:

$$
\begin{gathered}
E_{x}^{s}=-j \omega \cdot G_{x x}^{A} * J_{x}+\frac{1}{j \omega} \frac{\partial}{\partial x}\left[G_{x}^{q_{e}} * \frac{\partial}{\partial x} J_{x}+C_{i}^{q_{e}} * \frac{\partial}{\partial z} J_{z}\right] \\
E_{y}^{s}=-j \omega \cdot G_{y y}^{A} * J_{y} \\
E_{z}^{s}=-j \omega\left(G_{z x}^{A} * J_{x}+G_{z:}^{A} * J_{z}\right)+\frac{1}{j \omega} \frac{\partial}{\partial z}\left[C_{z}^{\prime q_{*}} * \frac{\partial}{\partial x} J_{x}+C_{z}^{\prime z *} * \frac{\partial}{\partial z} J_{z}\right]
\end{gathered}
$$

For the calculation of the incident field. a simple case in 2-D represented in Fig. 3.1 is considered. If the excitation is transverse electric (TE) to $y$. then the magnetic field is in $y$-direction, and written as

$$
\boldsymbol{H}_{t e}^{i}=\dot{y} H_{i}\left(\epsilon^{\jmath\left(k_{x} x+k_{z}: i\right)}+\dot{R}_{T, i}^{i, i-1} \epsilon^{\jmath\left(k_{x} x-k_{z}=\right)}\right)
$$



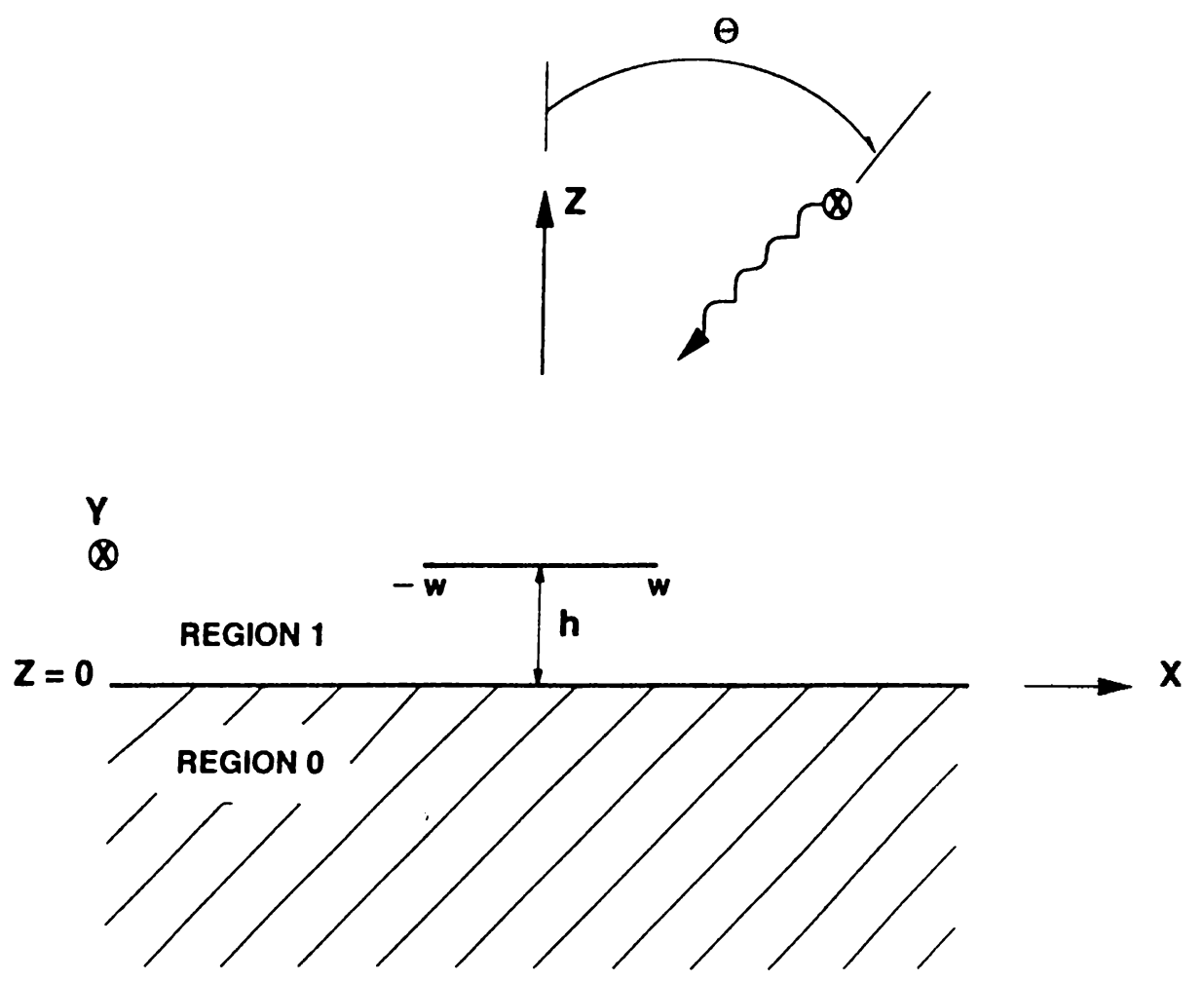

Figure 3.1: Strip of width $2 w$ located near interface between two semi-infinite half-spaces and illuminated by source in upper half-space

Since the electric field integral equation is used throughout this thesis. the incident electric field is obtained from

$$
\Gamma \times H_{t \epsilon}^{i}=j w^{\prime} \epsilon_{i} E_{t e}^{i}
$$

as

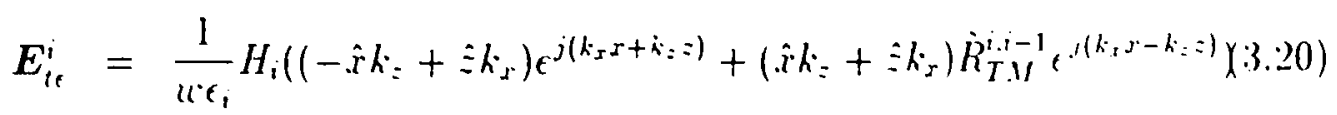

When the excitation is transverse magnetic (T.M) to $\mathrm{y}$. the electric ficld becomes in $y$ direction, and written as

$$
E_{t m}^{\prime}=\hat{y} E_{i}\left(c^{J\left(k_{x} x+k_{:}:\right)}+\dot{R}_{T E}^{\prime, i-1} \epsilon^{J\left(k_{z} r-k_{z}: z\right)}\right)
$$


Since most of the examples in the literature are given as the current density normalized with respect to the incident magnetic field. this field component needs to be determined. From the following Maxwell's equation

$$
\nabla \times E_{t m}^{i}=-j u \cdot \mu_{i} H_{t m}^{i}
$$

the incident magnetic field is obtained as

$$
\boldsymbol{H}_{t m}^{i}=\frac{-1}{u \mu_{i}} E_{i}\left(\left(-\hat{x} k_{z}+\hat{z} k_{x}\right) e^{j\left(k_{x} x+k_{z} z\right)}+\left(\hat{x} k_{z}+\hat{z} k_{x}\right) \dot{R}_{T E}^{i, i-1} \epsilon^{j\left(k_{x} x-k_{z}=1\right)} 03\right.
$$

where

$$
\begin{aligned}
& k_{i}=\sqrt{k_{x}^{2}+k_{z}^{2}} \\
& k_{x}^{\prime}=k_{i} \sin \theta \\
& k_{z}=k_{i} \cos \theta
\end{aligned}
$$

and $\dot{R}_{T E}^{i, i-1}$ and $\dot{R}_{T M}^{i, i-1}$ are given in Eq. (2.20).

\subsection{Basis and Testing Functions}

The Method of Moments converts an integral equation to a matrix equation and solves the matrix equation through the expansion of the unknown function in terms of known basis functions and weighting the resulting expression by the testing functions. Therefore. one needs to choose the basis and testing functions judiciously to minimize the error in average sense. Since the Galerkin method is used in the study: the basis and testing functions are chosen from the same set of functions.

In this section. the basis and testing functions that are used throughout this 
thesis are presented. The current densities for T.M and TE excitations are expanded in terms of sub-domain basis functions as

$$
\begin{aligned}
& J_{y}(v)=\sum_{m=1}^{M_{t m}} I_{y m} B_{y m}(v) \\
& J_{v}(v)=\sum_{m=1}^{M_{t e}} I_{v m} B_{v m}(v)
\end{aligned}
$$

and the testing functions $T_{n}(u)$ are used as in Fig. 3.2, where $u$ and $v$ are functions of $x$ and $z$.
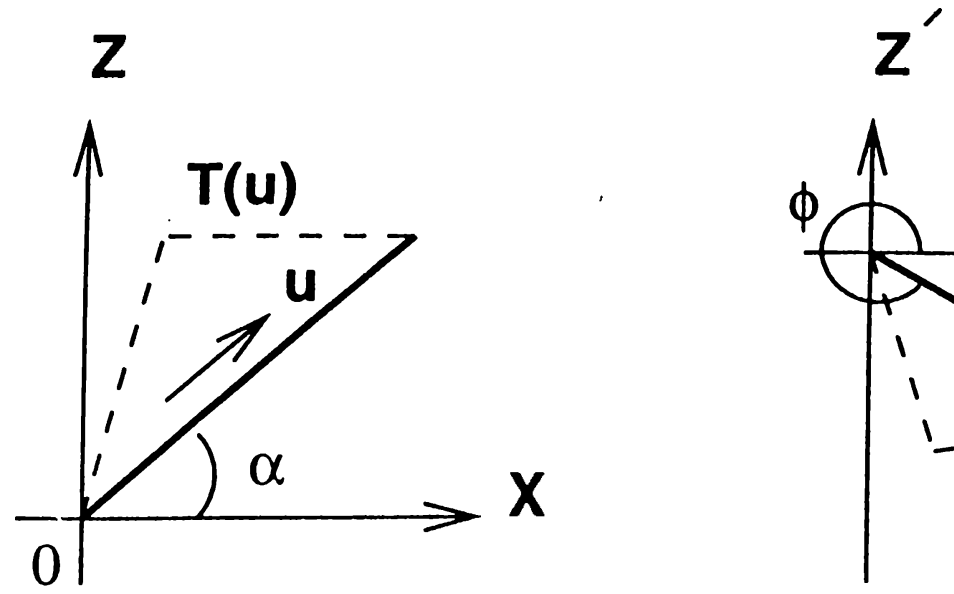

Figure 3.2: 0 and $\alpha$ on basis and testing functions

These testing and basis functions are chosen as triangular functions. Fig. 3.3. and given $b y$

$$
B(v)=\left\{\begin{array}{cl}
\frac{r-v_{1}}{\left(r_{2}-v_{1}\right)\left(r_{3}-r_{1}\right)} & \text { if } r_{1} \leq r \leq v_{2} \\
\frac{r_{3}-r}{\left(r_{3}-r_{2}\right)\left(r_{3}-r_{1}\right)} & \text { if } r_{2} \leq r \leq v_{3} \\
0 & \text { otherwise }
\end{array}\right.
$$



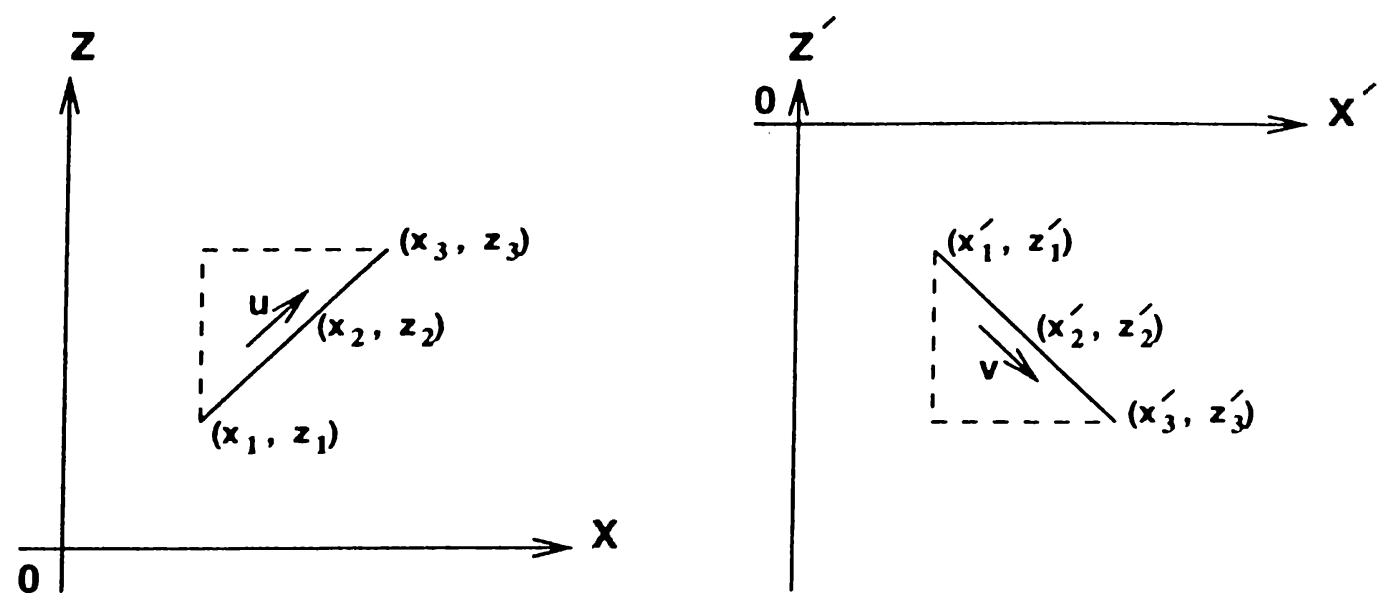

Figure 3.3: Single triangular testing and basis functions

$$
T(u)=\left\{\begin{array}{cl}
\frac{u-u_{1}}{\left(u_{2}-u_{1}\right)\left(u_{3}-u_{1}\right)} & \text { if } u_{1} \leq u \leq u_{2} \\
\frac{u_{3}-u}{\left(u_{3}-u_{2}\right)\left(u_{3}-u_{1}\right)} & \text { if } u_{2} \leq u \leq u_{3} \\
0 & \text { otherwise }
\end{array}\right.
$$

where

$$
\begin{gathered}
0=\arctan \left(\frac{z_{3}^{\prime}-z_{1}^{\prime}}{x_{3}^{\prime}-x_{1}^{\prime}}\right) \\
x^{\prime}=r \cos \phi \\
z^{\prime}=r \sin \dot{ }
\end{gathered}
$$

and

$$
\begin{gathered}
a=\arctan \left(\frac{z_{3}-z_{1}}{x_{3}-r_{1}}\right) \\
x=u \cos n \\
z=u \sin n
\end{gathered}
$$


Since both the basis and testing functions are in the same form. the Fourier transform of the testing function is given here as

$$
\tilde{T}\left(k_{u}\right)=\frac{-\left(u_{3}-u_{2}\right) \epsilon^{j k_{u} u_{1}}+\left(u_{3}-u_{1}\right) \epsilon^{j k_{u} u_{2}}-\left(u_{2}-u_{1}\right) \epsilon^{j k_{u} u_{3}}}{k_{u}^{2}\left(u_{2}-u_{1}\right)\left(u_{3}-u_{1}\right)\left(u_{3}-u_{2}\right)}
$$

to be used in the spatial domain applications of MoM.

\subsection{MoM Formulation in Spatial Domain}

The total electric field for TE excitation in region $i$ is the summation of the scattered electric fields and the incident electric field

$$
E_{t e}^{t}=\hat{x} E_{x}^{s}+\hat{z} E_{z}^{s}+E_{t \epsilon}^{i}
$$

Similarly, the total electric field for TM excitation is written as

$$
\boldsymbol{E}_{t m}^{t}=\hat{y} E_{y}^{s}+\boldsymbol{E}_{t m}^{i}
$$

Boundary conditions for the tangential electric fields for both TM and TE excitations are applied on the conducting body as

$$
\left(E_{t m}^{t}\right)_{t a n}=0 \quad \text { and } \quad\left(E_{t \varepsilon}^{t}\right)_{t a n}=0
$$

and substituting Eqs. (3.38) and (3.39) results in the following electric field integral equations (EFIE):

$$
\begin{aligned}
\left(\hat{x} E_{x}^{s}+\hat{z} E_{z}^{s}\right)_{t a n} & =-\left(E_{t e}^{i}\right)_{t a n} \\
\left(\hat{y} E_{y}^{s}\right)_{t a n} & =-\left(E_{t m}^{i}\right)_{t a n}
\end{aligned}
$$

With the application of the testing procedure of the Moll to these integral equalions

$$
\left\langle T_{\imath n}(u) . \hat{r} E_{s}^{s}+\vdots E_{s}^{s}\right\rangle=-\left\langle T_{\imath n}(u) . E_{t !}^{i}\right\rangle
$$


for TE excitation. and

$$
\left\langle T_{y n}(u), E_{y}^{s}\right\rangle=-\left\langle T_{y n}(u) \cdot E_{t m}^{i}\right\rangle
$$

for TM excitation are obtained. Using the testing function of

$$
\begin{aligned}
\mathbf{T}_{\mathbf{v n}}(u) & =\hat{u} T_{v n}(u) \\
& =(\hat{x} \cos \alpha+\hat{z} \sin \alpha) T_{\imath n}(u)
\end{aligned}
$$

for TE case, the left hand side of Eq. (3.43) becomes

$$
\begin{aligned}
& \left\langle\mathbf{T}_{\mathbf{v n}}(u), \hat{v} E_{z}^{s}\right\rangle=\left\langle\mathbf{T}_{\mathbf{v n}}(u), \hat{x} E_{x}^{s}+\hat{z} E_{z}^{s}\right\rangle \\
& =\int d u\left(\cos \alpha E_{x}^{s}+\sin \alpha E_{z}^{s}\right) T_{v^{\prime n}}(u) \\
& =-j u \sum_{m=1}^{M_{t e}} I_{v m}\left[\int d u T_{\imath n}(u)\left(\cos a \cos o G_{x x}^{A} * B_{1 \cdot m}(v)\right)\right. \\
& +\int d u T_{v n}(u)\left(\sin \alpha \cos o G_{* r}^{A} * B_{\imath m}(v)\right) \\
& \left.+\int d u T_{v n}(u)\left(\sin 0 \sin \circ G_{z:}^{A} * B_{u m}(v)\right)\right] \\
& -\frac{1}{j w}\left[\int d u \frac{d T_{v n}(u)}{d u}\left(\cos ^{2} \circ C_{x}^{\eta \kappa} * \frac{d B_{v m}(v)}{d v}\right)\right. \\
& \left.+\int d u \frac{d T_{v^{\prime n}}(u)}{d u}\left(\sin ^{2} \phi C_{*}^{* u} * \frac{d B_{r^{\prime m}}(v)}{d v}\right)\right]
\end{aligned}
$$

and. the right hand side becomes

$$
\begin{aligned}
& \left\langle\mathbf{T}_{\mathbf{v n}}(u) \cdot \boldsymbol{E}^{i}\right\rangle=-\frac{k_{i} H_{i}}{u \epsilon_{i}}\left[\int d u \cos (\alpha+\theta) T_{r n}(u) \epsilon^{j\left(k_{x} x+k_{:}=1\right.}\right. \\
& \left.-\int d u \cos (\alpha-\theta) \dot{R}_{T M}^{i_{i}, i-1} T_{\imath n}(u) \epsilon^{\jmath\left(k_{x} r-k_{z}:\right)}\right] \\
& =-\frac{k_{i} H_{i}}{u \epsilon_{i}}\left[\cos (\alpha+\theta) \dot{T}_{r n}(k \sin (\alpha+\theta)) \epsilon^{\prime\left(k_{x} x_{n_{2}}+k_{z}=n_{2}\right)}\right. \\
& \left.-\dot{R}_{T M}^{i, i-1} \cos (\alpha-\theta) \grave{T}_{i n}(k \sin (\theta-\Omega)) e^{j\left(k_{2}, r_{n_{2}}-k_{z}=n_{2}\right)}\right)(3
\end{aligned}
$$

Similarly: using the testing function of

$$
\mathbf{T}_{\mathbf{y n}}(u)=\dot{y} T_{y n}(u)
$$


for T.M case, the left hand side of Eq. (3.44) becomes

$$
\left\langle\mathbf{T}_{\mathbf{y n}}(u), \hat{y} E_{y}^{s}\right\rangle=-j u \cdot \sum_{m=1}^{M_{t m}} I_{y m} \int d u T_{y n}(u) C_{y y}^{A} * B_{y m}(v)
$$

and the right hand side becomes

$$
\begin{aligned}
\left\langle\mathbf{T}_{\mathbf{y n}}(u), \boldsymbol{E}^{i}\right\rangle= & \int d u T_{y n}(u) E_{i}\left(e^{j\left(k_{x} x+k_{z} z\right)}+\dot{R}_{T E}^{i, i-1} \epsilon^{j\left(k_{x} x-k_{z}=\right)}\right) \\
= & E_{i}\left(\dot{T}_{y n}(k \sin (\alpha+\theta)) \epsilon^{j\left(k_{x} x_{n_{2}}+k_{z}=n_{2}\right)}\right. \\
& \left.+\tilde{R}_{T E}^{i, i-1} \tilde{T}_{y n}(k \sin (\theta-\alpha)) \epsilon^{j\left(k_{x} x_{n_{2}}-k_{z} z_{n_{2}}\right)}\right)
\end{aligned}
$$

Since Green's functions in the spatial domain are approximated as the summation of the Hankel functions of second kind. for small arguments they grow indefinitely and the integrals cannot be calculated efficiently. In order to solve this problem, a singularity extraction method must be used for small arguments.

\subsubsection{Singularity Extraction}

The zeroth order Hankel function of the second kind $H_{o}^{(2)}(k, \rho)$ has a logarithmic singularity for the argument $k_{i} \rho \rightarrow 0$.

$$
H_{0}^{(2)}\left(k_{i} \rho\right) \sim \frac{2 j}{\pi} \ln \left(k_{i} \rho\right)
$$

Therefore, in the calculation of Eqs. (3.46) and (3.50) for $|n-m| \leq 1$. the singularity extraction needs to be performed in order to find the self term $(n=m)$ and the interaction between neighboring basis and testing functions.

To demonst rate the singularity extraction method on a simple example. a horizont al strip in a homogeneous medium is considered. Fig. 3.4. For TM excitation. Green's function is

$$
G_{y, y}^{A}=-\frac{j \mu_{1}}{1} H_{0}^{(2)}\left(k_{i} \rho\right)
$$




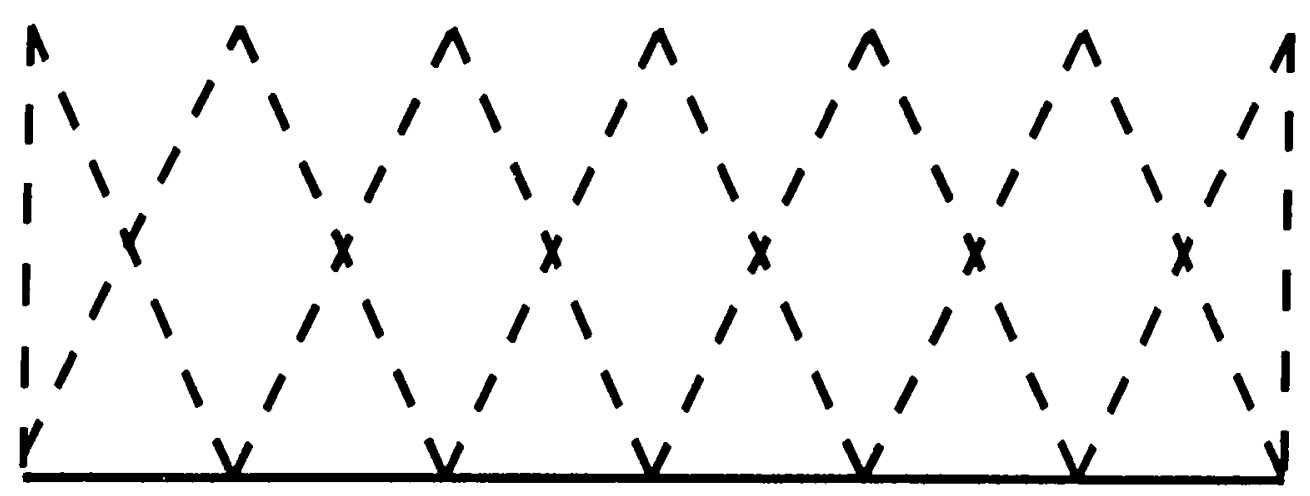

- W

w

Figure 3.4: Basis and testing functions on a single horizont al strip of width $2 w$. so the left hand side of the MoM equation (3.50) becomes

$$
\left\langle\mathbf{T}_{\mathbf{y n}}(u), \hat{y} E_{y}^{s}\right\rangle=-\frac{\dot{\alpha} \mu_{i}}{4} \sum_{m=1}^{M_{t m}} I_{y m} \int d u T_{y n}(u) \int d v \cdot H_{0}^{(2)}\left(k_{i} \rho\right) B_{y n u}(l \cdot) \cdot(3)
$$

I'sing the following notation:

$$
\begin{aligned}
& x_{n m}=x_{n_{2}}-x_{m_{2}} \\
& z_{n m}=z_{n_{2}}-z_{m_{2}} \\
& z_{n m}^{\prime}=z_{n_{2}}+z_{m_{2}}
\end{aligned}
$$

where $r_{n_{2}}, z_{n_{2}}, x_{m_{2}}$ and $z_{m_{2}}$ are the mid-point coordinates of the testing and basis functions, respectively, $\rho$ can be defined as

$$
\begin{aligned}
\rho & =\sqrt{\left(x-x^{\prime}\right)^{2}+\left(z-z^{\prime}\right)^{2}} \\
& =\sqrt{\left(x_{n m}+u \cos a-v \cos O\right)^{2}+\left(z z_{n}+u \sin O-v \sin O\right)^{2}} \\
& =\sqrt{k(u, r)} \\
& =\sqrt{r^{2}+B r+C}
\end{aligned}
$$

Where

$$
B=-2\left(x_{n m} \cos \phi+z z_{m} \sin \phi+11 \cos (\alpha-0)\right)
$$




$$
C=x_{n m}^{2}+z_{n m}^{2}+u^{2}+2 u\left(x_{n m} \cos a+z_{n m} \sin a\right)
$$

To circumvent the difficulty in numerical integration of $\mathrm{Eq}$. (3.5.5) due to the singularity of the Hankel function. one can subtract and add the small argument form of the Hankel function from Green's function. resulting in

$$
\begin{aligned}
\left\langle\mathbf{T}_{\mathbf{y z}}(u), \hat{y} E_{y}^{s}\right\rangle= & -j u \cdot \sum_{m=1}^{M_{t m}} I_{y m} \int d u T_{y n}(u) \int d v\left(H_{0}^{(2)}\left(k_{i} \rho\right)-\frac{2 j}{\pi} \ln \left(k_{i} \rho\right)\right) B_{y m}(v) \\
& -j u \cdot \sum_{m=1}^{M_{t m}} I_{y m} \int d u T_{y n}(u) \int d v \frac{2 j}{\pi} \ln \left(k_{i} \rho\right) B_{y m}(v)
\end{aligned}
$$

where the second integral on the right hand side can be evaluated analytically:

For an inhomogeneous case, since Green's function consists of a direct term plus four summations, Eq. (2.40). one needs to find the terms that can blow up for certain set of testing and basis functions and to use the same procedure as in the homogeneous case described above to extract singularities.

\subsection{MoM Formulation using FFT}

The discrete Fourier transform (DFT) plays an important role in the analysis. design and implementation of discrete-time signal processing algorithms and sysstems. The DFT is identical to samples of the Fourier transform at equally spaced frequencies. Consequently, computation of the R-point DFT corresponds to the computation of $R$ samples of the Fourier transform at $R$ equally spaced frequencies. The efficient algorithms which calculate DFT are called fast Fourier transform (FFT) algorithms [1-1].

FFT algorithms are based on the fundamental principle of decomposing the computation of the DFT of a sequence of length $R$ into successively smaller discrete Fourier transforms. The manner in which this principle is implemented 
leads to a variety of different algorithms, all with comparable improvements in computational speed. In terms of multiplications and additions. the class of FFT algorithms can be orders of magnitude more efficient than competing algorithms. The efficiency of FFT algorithms is so high. in fact, that in many cases the most efficient procedure for implementing a convolution is to compute the transform of the sequences to be convolved, multiply their transforms, and then compute the inverse transform of the product of transforms.

As previously mentioned. if the EFIE is written in the spectral domain. the convolution between Green's function and the basis function becomes multiplication, so the double integral of the MoM equation is reduced to a single integral. In this section, the application of FFT to the evaluation of this single integral will be explained for planar geometries only:

The scattered electric fields for TE and TM excitations can be written for a planar geometry (printed on $x-y$ plane) as

$$
\begin{gathered}
E_{x}^{s}=-j \omega \cdot G_{x x}^{A} * J_{x}+\frac{1}{j \cdot} \cdot \frac{\partial}{\partial x}\left(G_{x}^{q_{e}} * \frac{\partial}{\partial x} J_{x}\right) \\
E_{y}^{s}=-j \cdot \cdot\left(G_{y y}^{A} * J_{y}\right.
\end{gathered}
$$

and their Fourier transforms are

$$
\begin{aligned}
& \dot{E}_{x}^{s}=-j_{\omega} \cdot \dot{C}_{r x}^{E} j_{x}\left(k_{x}\right) \\
& \dot{E}_{y}^{s}=-j_{u} \cdot \dot{G}_{y y}^{t} \dot{j}_{y}\left(k_{x}\right)
\end{aligned}
$$

where

$$
\dot{C}_{r s}^{E}=\dot{G}_{r r}^{4}-\frac{k_{r}^{2}}{i^{2}} \dot{C}_{r}^{q r}
$$


Since the strips are horizontal. we only need the incident ficld in $x$-direction for TE excitation and in $y$-direction for T.M excitation.

$$
\begin{aligned}
& E_{x}^{i}=-\frac{k \cos \theta H_{i}}{\dot{i \epsilon}} \epsilon^{j k \sin \theta x}\left(\epsilon^{j k \cos \theta z}-\dot{R}_{T M}^{i, i-1} \epsilon^{j k \cos \theta z}\right) \\
& E_{y}^{i}=E_{i} \epsilon^{j k \sin \theta x}\left(\epsilon^{j k \cos \theta z}+\dot{R}_{T E}^{i, i-1} \epsilon^{j k \cos \theta z}\right)
\end{aligned}
$$

and their Fourier transforms are

$$
\begin{aligned}
\dot{E}_{x}^{i} & =-\frac{k \cos \theta H_{i}}{\omega \epsilon} 2 \pi \delta\left(k_{x}+k \sin \theta\right)\left(\epsilon^{j k \cos \theta z}-\dot{R}_{T M}^{i, i-1} \epsilon^{-j k \cos \theta z}\right) \\
\dot{E}_{y}^{i} & =E_{i} 2 \pi \delta\left(k_{x}+k \sin \theta\right)\left(\epsilon^{j k \cos \theta z}+\dot{R}_{T E}^{i, i-1} \epsilon^{-j k \cos \theta z}\right) .
\end{aligned}
$$

Since the total electric fields are the summation of scattered and incident electric fields, their Fourier transforms are given for TE and TM excitation as

$$
\begin{aligned}
& \dot{E}_{x}^{t}=\dot{E}_{x}^{s}+\dot{E}_{x}^{i} \\
& \dot{E}_{y}^{t}=\dot{E}_{y}^{s}+\dot{E}_{y}^{i}
\end{aligned}
$$

The basis and testing functions are chosen as triangular functions for the TE excitation :

$$
\begin{gathered}
B_{x}(x)=\left\{\begin{array}{cl}
\frac{x-x_{1}}{h_{x}} & \text { if } x_{1} \leq x \leq x_{2} \\
\frac{x_{3}-x}{h_{x}} & \text { if } x_{2} \leq x \leq x_{3} \\
0 & \text { otherwise }
\end{array}\right. \\
\dot{B}_{x}\left(k_{x}\right)=\frac{4}{h_{x} k_{x}^{2}} \epsilon^{j k_{x} x_{2}} \sin ^{2}\left(\frac{k_{x} h_{x}}{2}\right) \\
=h_{x} \epsilon^{j k_{x} x_{2}} \operatorname{sinc}^{2}\left(\frac{k_{x} h_{x}}{2}\right)
\end{gathered}
$$

and. pulse functions for the TM excitation

$$
B_{y}(x)= \begin{cases}1 & \text { if } x_{1} \leq x \leq x_{3} \\ 0 & \text { otherwise }\end{cases}
$$




$$
\begin{aligned}
\dot{B}_{y}\left(k_{x}\right) & =\frac{2}{k_{x}} \epsilon^{j k_{x} x_{2}} \sin \left(\frac{k_{x} h_{x}}{2}\right) \\
& =h_{x} \epsilon^{j k_{x} x_{2}} \sin c\left(\frac{k_{x} h_{x}}{2}\right)
\end{aligned}
$$

where $x_{2}-x_{1}=x_{3}-x_{2}=h_{x}$. Consequently, the application of the boundary condition on the total electric fields Eqs. (3.72) and (3.73) via the testing procedure results in the following set of linear equations: For TE excitation

$$
\left.-j w \sum_{m=1}^{M_{\text {te }}} I_{x m}\left\langle\dot{T}_{x n}^{*}, \dot{G}_{x x}^{E} \dot{B}_{x m}\right\rangle=-<\tilde{T}_{x n}^{*}, \tilde{E}_{x}^{i}\right\rangle \text { for } n=1 \ldots, M I_{\text {te }}
$$

where $I_{x m}$ 's are the unknown coefficients of the basis functions to be determined. and each term can be written explicitly as

$$
\left\langle\dot{T}_{x n}^{*} \cdot \dot{G}_{x x}^{E} \dot{B}_{x m}\right\rangle=\int_{-\infty}^{\infty} d k_{x}^{\prime} \epsilon^{-j k_{x}\left(x_{n_{2}}-x_{m_{2}}\right)} h_{x}^{2} \operatorname{sinc}^{4}\left(\frac{k_{x} h_{x}}{2}\right) \dot{G}_{x x}^{E}
$$

and

$$
\begin{aligned}
-<\dot{T}_{r n}^{*} . \dot{E}_{x}^{i}>= & \frac{k \cdot \cos \theta H_{i}}{\omega \epsilon} 2 \pi \epsilon^{j k \sin \theta n_{n_{2}}}\left(\epsilon^{j k \cos \theta=}-\dot{R}_{T M}^{i, i-1} \epsilon^{j k \cos \theta_{z}}\right) \times \\
& h_{x} \sin c^{2}\left(\frac{k \sin \theta h_{x}}{2}\right)
\end{aligned}
$$

For T.I excitation

$$
\left.-j u \cdot \sum_{m=1}^{M_{z m}} I_{y m}\left\langle\dot{T}_{y n}^{*}, \dot{G}_{y y}^{+} \dot{B}_{y m}\right\rangle=-<\dot{T}_{y n}^{*}, \dot{E}_{y}^{i}\right\rangle \quad \text { for } n=1 \ldots \ldots M_{t m}
$$

where $I_{y m}$ 's are the unknown coefficients of the basis functions to be determined. and each term can be written explicitly as

$$
j u\left\langle\left\langle\dot{T}_{y n_{i}}^{*} \cdot \dot{G}_{y y}^{A} \dot{B}_{y m}\right\rangle=j u \cdot \int_{-x}^{x} d k_{x} c^{-j k_{x}\left(r_{n_{2}}-r_{m_{2}}\right)} h_{x}^{2} \sin c^{2}\left(\frac{k_{x} h_{x}}{2}\right) \dot{c}_{y y}^{A}\right.
$$

and

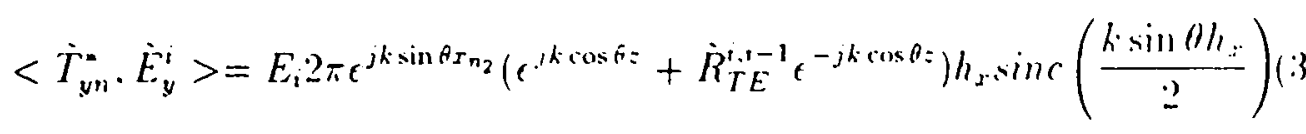


To demonstrate the application of FFT to the calculation of the Mo.M matrix entries given above, only the case for the TE excitation is given here in detail. as the case for the T.M excitation is the same as that of the TE excitation. If the matrix entries in Eq. (3.79) are examined, it is seen that the exponential term acts as the kernel of the transformation and the rest is the function to be transformed, given as

$$
h\left(k_{x}\right)=\operatorname{sinc} c^{4}\left(\frac{k_{x} h_{x}}{2}\right) \tilde{G}_{x x}^{E}
$$

where $k_{x}$ is bounded between $-K$ and $K$. Therefore. the matrix entries can be re-written as

$$
\left\langle\dot{T}_{x n}^{*}, \dot{G}_{x x}^{E} \dot{B}_{x m}\right\rangle=\check{h}_{x}^{2} \int_{-K}^{K} d k_{x} \epsilon^{j k_{x}\left(x_{m_{2}}-x_{n_{2}}\right)} h\left(k_{x}\right)
$$

which is further converted to

$$
\begin{aligned}
\int_{-K}^{K} d k_{x} \epsilon^{j k_{x} d} h\left(k_{x}\right) & =e^{-j k d} \int_{0}^{2 K} d k_{r}^{\prime} \epsilon^{j k_{x}^{\prime} d} h\left(k_{x}^{\prime}-K^{\prime}\right) \\
& \left.=\epsilon^{-j K d} \sum_{r=1}^{R} h\left(k_{x_{r}}^{\prime}-K^{\prime}\right) \epsilon^{j k_{x_{r}}^{\prime} d}\right\lrcorner=I(d)
\end{aligned}
$$

where $d=x_{m_{2}}-x_{n_{2}} \cdot k_{r_{r}}^{\prime}=(r-1) \Delta$ and $\Delta=2 K / \Lambda$.

A fast Fourier transform routine from a scientific library IMSL. DFFTC'F. is used to compute the discrete complex Fourier transform of a complex vector of size $R$. The method used is a variant of the Cooley-Tukey algorithm. which is most efficient when $R$ is a product of small prime factors. If $R$ satisfies this condition, then the computational effort is proportional to $R \log R$. 'This considerable saving has historically led people to refer to this algorithm as the 'fast Fourier transform or FFT. Specifically given an $R$-vector $x$. DFFTC'F returms in $\mathrm{c}=\mathrm{COEF}$

$$
c_{s}=\sum_{r=1}^{R} x_{r} e^{-2 r i(r-1)(s-1) / R} \text { for } s=1 \ldots . R
$$


Ising this formula, $I(d)$ can be written as

$$
I(m)=I((m-1) \Delta d)=\epsilon^{-j k(m-1) \Delta d} \sum_{r=1}^{R} h\left(k_{x_{r}}^{\prime}-K\right) \epsilon^{j 2 \pi(r-1)(s-1) / R} \Delta
$$

where $\Delta d=\pi / h^{\prime}$ and $d=(m-1) \Delta d$, or

$$
I(m)=e^{-j \pi(m-1)} \Delta \sum_{r=1}^{R} h\left(k_{x_{r}}^{\prime}-K\right) e^{j 2 \pi(r-1)(s-1) / R} \quad s=1.2 \ldots R R
$$

which is the FFT of $h\left(k_{x}\right)$ of size $R$, but, we only need $\eta_{t \epsilon}$ of this sequence since the number of basis functions is $M_{t e}$. To obtain the matrix entries. the exponents in Eq. (3.8T) and (3.90) are compared,

$$
k_{x_{r}}^{\prime} d=2 \pi(r-1)(s-1) / R
$$

where $d=x_{m_{2}}-x_{n_{2}}=(n-1) h_{r} \cdot k_{x_{r}}^{\prime}=(r-1) \Delta$. so

$$
\left.k_{x_{r}}^{\prime}\left(x_{m_{2}}-x_{n_{2}}\right)=(n-1)(r-1) h_{x}\right\rfloor=2 \pi(r-1)(s-1) / R
$$

is obtained. which results in

$$
s=\frac{(n-1) h_{x} R د}{2 \pi}+1
$$

From this relation, one can easily find the terms corresponding to the matrix entries from the FFT results.

\subsection{MoM Formulation in Spectral Domain}

In the previous section, the Mo.M matrix entries, which are single integrals. were calculated by using FFT. i.e. the integral was converted to summation and the evaluation was performed via the FFT algorithm.

In this section, a new approach is developed to find the Mo.l matrix cutries, in which the whole integrand. except the kernel. are approximated in terms of 
complex exponentials, and the inverse Fourier transforms of these exponentials are found analytically. The procedure is demonstrated for the case of the TE excitation, for which the MoM matrix entries are given as

$$
\left\langle\dot{T}_{x n}^{*}, \dot{G}_{x x}^{E} \tilde{B}_{x m}\right\rangle=\int_{-\infty}^{\infty} d k_{x} e^{-j k_{x}\left(x_{n_{2}}-x_{m_{2}}\right)} h_{x}^{2} \sin c^{4}\left(\frac{k_{x} h_{x}}{2}\right) \dot{G}_{x x}^{E}
$$

where

$$
\begin{aligned}
\hat{G}_{x x}^{E}= & \dot{G}_{x x}^{A}-\frac{k_{x}^{2}}{\omega^{2}} \dot{G}_{x}^{q_{e}} \\
\dot{G}_{x x}^{E}= & \frac{k_{z_{1}}}{j 2 k_{i}^{2}}\left\{\epsilon^{-j k_{z_{1}}\left|z-z^{\prime}\right|}-M_{i}^{T M M} \dot{R}_{T M}^{i, i+1} \epsilon^{-j k_{z_{2}} 2 d_{1}} \epsilon^{j k_{z_{1}}\left(z+z^{\prime}\right)}\right. \\
& +M_{i}^{T M} \dot{R}_{T M}^{i, i-1} \hat{R}_{T M}^{i, i+1} \epsilon^{-j k_{z_{1}} 2 d_{1}} \epsilon^{j k_{z_{1}}\left(z-z^{\prime}\right)}-M_{i}^{T M} \dot{R}_{T M}^{i, i-1} \epsilon^{-j k_{z_{1}}\left(z+z^{\prime}\right)} \\
& \left.+M_{i}^{T M} \tilde{R}_{T M}^{i, i-1} \hat{R}_{T M}^{i, i+1} \epsilon^{-j k_{z_{2}} 2 d_{1}} \epsilon^{-j k_{z_{1}}\left(z-z^{\prime}\right)}\right\} \\
= & A_{0} \epsilon^{-j k_{z_{1}}\left|=-z^{\prime}\right|}+A_{1} \epsilon^{j k_{z_{1}}\left(z+z^{\prime}\right)}+A_{2} \epsilon^{j k_{z_{1}}\left(=-z^{\prime}\right)}+A_{3} \epsilon^{-j k_{z_{1}}\left(z+z^{\prime}\right)} \\
& +A_{2} \epsilon^{-j k_{z_{1}}\left(z-z^{\prime}\right)}
\end{aligned}
$$

Defining a set of new coefficients like

$$
A_{l}^{\prime}=j 2 k_{z=} \sin c^{4}\left(\frac{k_{x} h_{x}}{2}\right) \cdot A_{l} \quad \text { for } 1=0 \ldots .3
$$

Eq. (3.94) can be written as

$$
\begin{aligned}
& \left\langle\dot{T}_{x n}^{*} \cdot \dot{C}_{x x}^{E} \dot{B}_{x m}\right\rangle=\int_{-\infty}^{\infty} d k_{x} \epsilon^{-j k_{x}\left(x_{n_{2}}-s_{m_{2}}\right)} \frac{h_{x}^{2}}{j \geq k_{z=1}}\left\{A_{0}^{\prime} \epsilon^{-j k_{z}\left|1:-\Sigma^{\prime}\right|}\right. \\
& +A_{1}^{\prime} \epsilon^{j k_{z_{1}}\left(z+z^{\prime}\right)}+A_{2}^{\prime} \epsilon^{j k_{z}\left(:-z^{\prime}\right)}+A_{3}^{\prime} \epsilon^{-j k_{z_{1}}\left(z+z^{\prime}\right)} \\
& \left.+A_{2}^{\prime} \epsilon^{-j k_{i_{1}}\left(=-z^{\prime}\right)}\right\}
\end{aligned}
$$

where $A_{l}^{\prime}$ s are approximated in terms of complex exponentials. Hence, the matrix entriss are evaluated analỵtically as

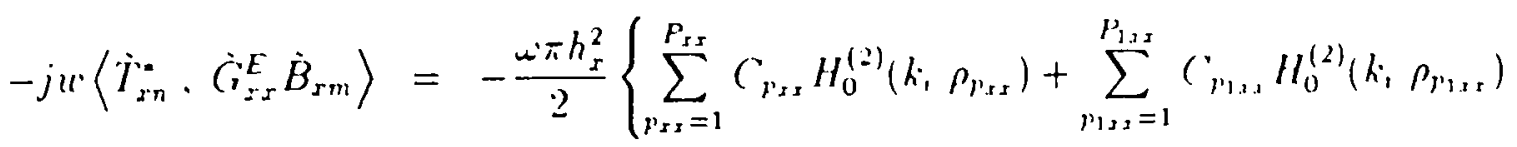




$$
\begin{aligned}
& +\sum_{p_{2 x x}=1}^{P_{2 x x}} C_{p_{2 x x}} H_{0}^{(2)}\left(k_{i} \rho_{p_{2 x}}^{(1)}\right)+\sum_{p_{3 x x}=1}^{P_{3 x x}} C_{p_{y_{x} x}} H_{0}^{(2)}\left(k_{i} \rho_{p_{3 x x}}\right) \\
& \left.+\sum_{p_{2 x x}=1}^{P_{2 x x}} C_{p_{2 x x}} H_{0}^{(2)}\left(k_{i} \rho_{p_{2 x x}}^{(2)}\right)\right\}
\end{aligned}
$$

where

$$
\begin{aligned}
\rho_{p_{x x}} & =\sqrt{\left(x_{n_{2}}-x_{m_{2}}\right)^{2}+\left(z-z^{\prime}\right)^{2}} \\
\rho_{p_{1 x x}} & =\sqrt{\left(x_{n_{2}}-x_{m_{2}}\right)^{2}+\left(z+z^{\prime}+j a_{p_{1 x x}}\right)^{2}} \\
\rho_{p_{2 x x}}^{(1)} & =\sqrt{\left(x_{n_{2}}-x_{m_{2}}\right)^{2}+\left(z-z^{\prime}+j a_{\left.p_{2 x x}\right)^{2}}\right.} \\
\rho_{p_{2 x x}}^{(2)}=\sqrt{\left(x_{n_{2}}-x_{m_{2}}\right)^{2}+\left(z-z^{\prime}-j a_{p_{2 x}}\right)^{2}} & \sqrt{\left(x_{n_{2}}-x_{m_{2}}\right)^{2}+\left(z+z^{\prime}-j a_{\left.p_{3 x}\right)^{2}}\right.}
\end{aligned}
$$

The advant ages of this approach is that. since the terms $A_{l}^{\prime}$ s are independent of $m$ and $n$. they are approximated only once and all the elements of impedance matrix are obtained from the closed form representation. Eq. (3.99). In addition. since Eq. (3.99) has z and z' explicitly, adding new strips or extending the sizes of the strips do not require any further manipulations. one just needs to eraluate Eq. (3.99) for different $z$ and $z^{\prime}$. or different $m$ and $n$. respectively. 


\section{Chapter 4}

\section{Numerical Examples}

In this chapter, some results obtaired using the three approaches derived in Chapter 3, namely the Method of Moments in the spectral and the spatial domains. and the FFT algorithm, will be given. As it was mentioned in Chapter 3. the spectral domain and the FFT approaches were formulated for horizontal strips and the spatial domain formulation can be applied to arbitrary surfaces in 2-D. However, in layered media. the spatial domain formulation can not be applied to tilted surfaces for TE excitation case because Green's functions are defined only for $x$ and $=$ directions of the current. In the first section of this chapter. the results of the spatial domain approach are given. The next section includes the CPU time comparison between the spatial, spectral domains and the FFT approaches for some examples. In the last section, examples of the spectral domain approach are provided. 


\subsection{Examples for the MoM Formulation in the Spatial Domain}

\subsubsection{A Single Horizontal Strip}
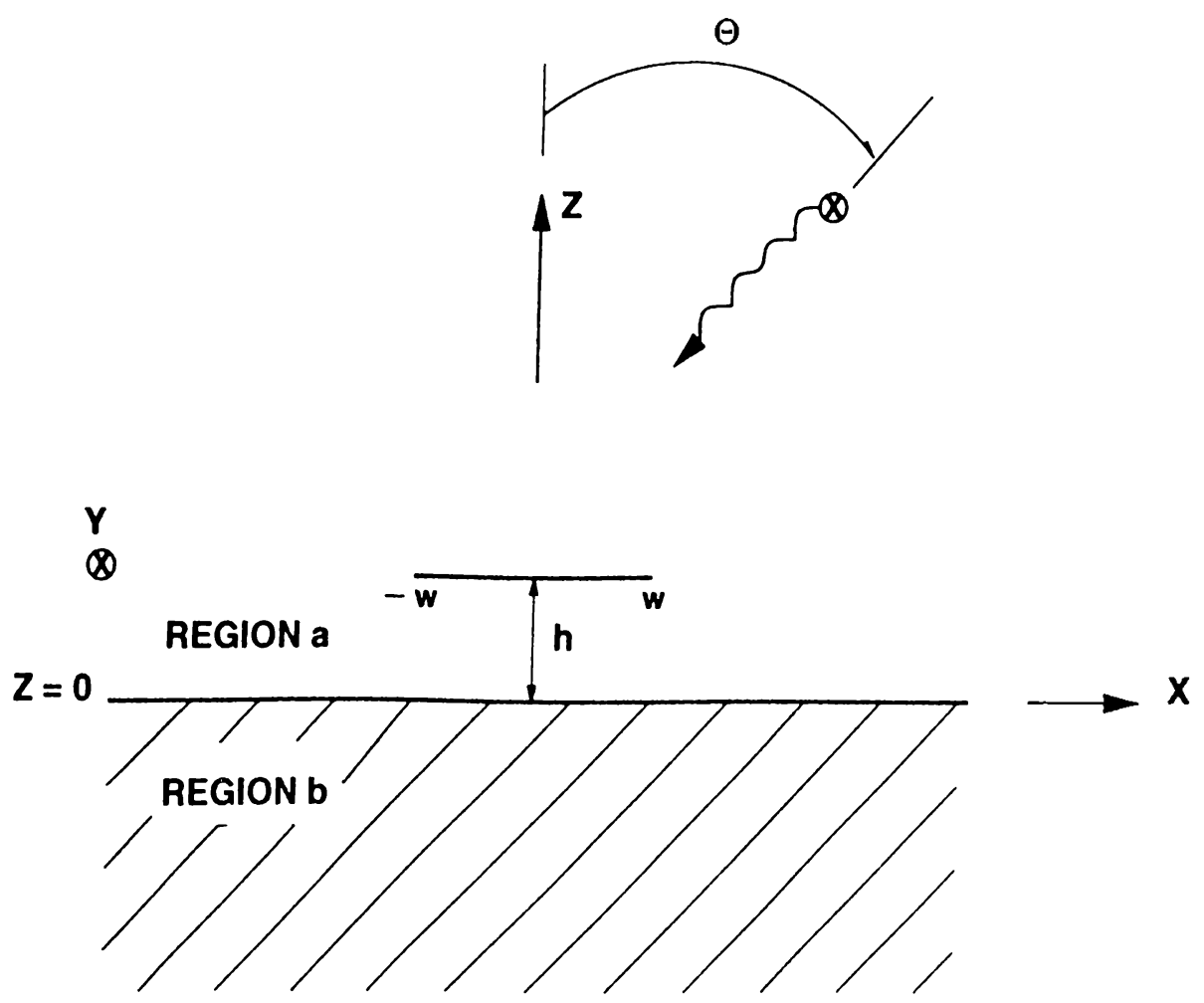

Figure 4.1: Strip of width $2 w$ located near interface between two semi-infinite half-spaces and illuminated by source in upper half-space

The spatial-domain $\mathrm{Mo.M}$ is first applied to a single horizontal strip located near the interface of two semi-infinite half-space. Fig. 4.1. The following parameters have been chosen for this example: the dielectric constants of Region $a$ and $b$. $t_{r_{a}}=1$ and $\epsilon_{r_{b}}=4:$ the width of the strip, $2 u=4 \lambda_{a}$ : the distance of the strip

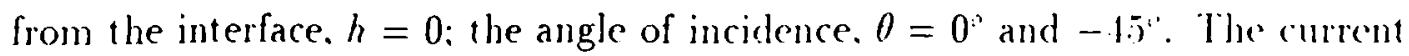
densities on the strip for the TM excitation are obtained for two different angles 
of incidence, Fig. 4.3, and compared to the results found in the literature [1:]. Fig. 4.2 note that $z$-axis in [15] corresponds to $y$-axis in our study. It js observed that the agreement is excellent, but to validate the code. another example on the same geometry is given for different values of $h .-0.1 \lambda_{b},-0.2 \lambda_{b}$ and $-0.3 \lambda_{b}$ and for $\theta=0^{\circ}$. For this example, the results are obtained for both TM and TE excitations, Figs. 4.5 and 4.7 , respectively, and compared to the results of Refs. [16], [17], reproduced in Figs. 4.4 and 4.6. Excellent agreement is again observed between the current distributions obtained by the spatial-domain MoM and found in the literature, except for, a slight derivation near the edges for T.I excitations, which might be due to the difference in number and form of the basis functions used in this study and in the literature. 


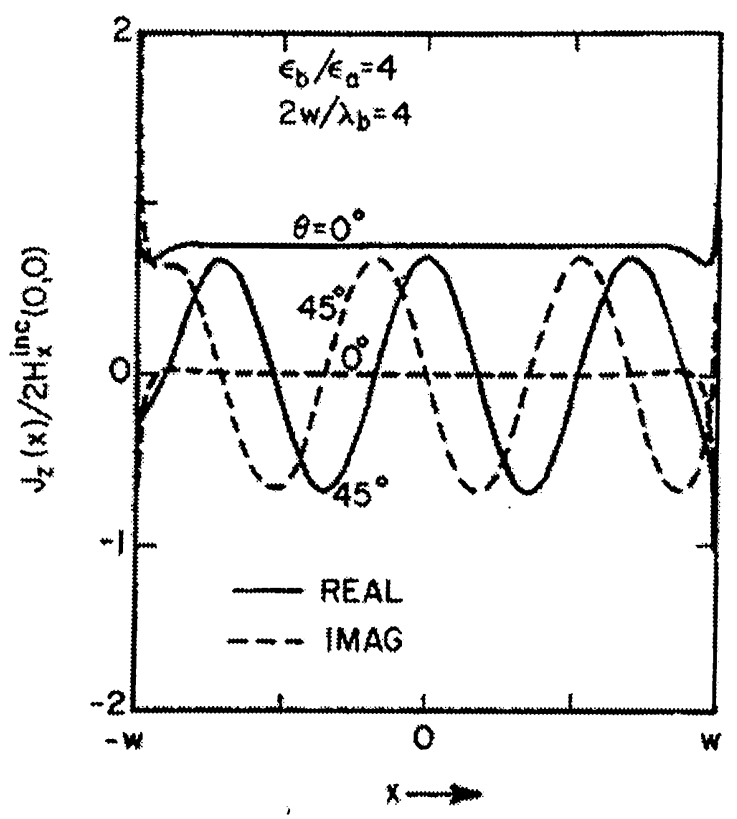

Figure 4.2: Normalized current density on a four wavelength strip for the TM excitation (reproduced from [15]). See Fig. 1.1 and the following text, for the geometry and its parameters.

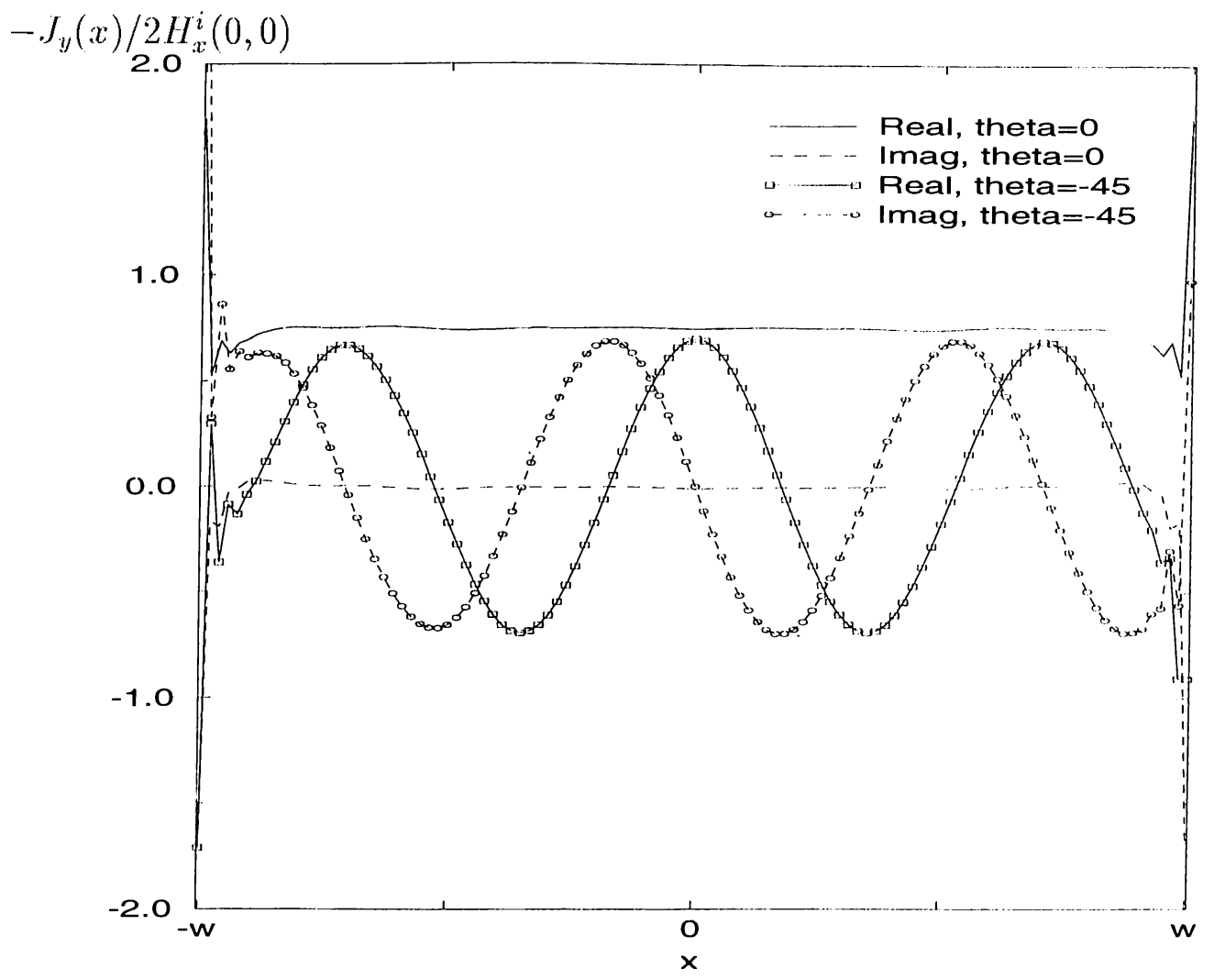

Figure 4.3: Normalized current density on a four-wavelength strip from the spatial-domain MoM for the I'M excitation. See Fig. 4.1 and tho following text for the geometry and its parameters. 


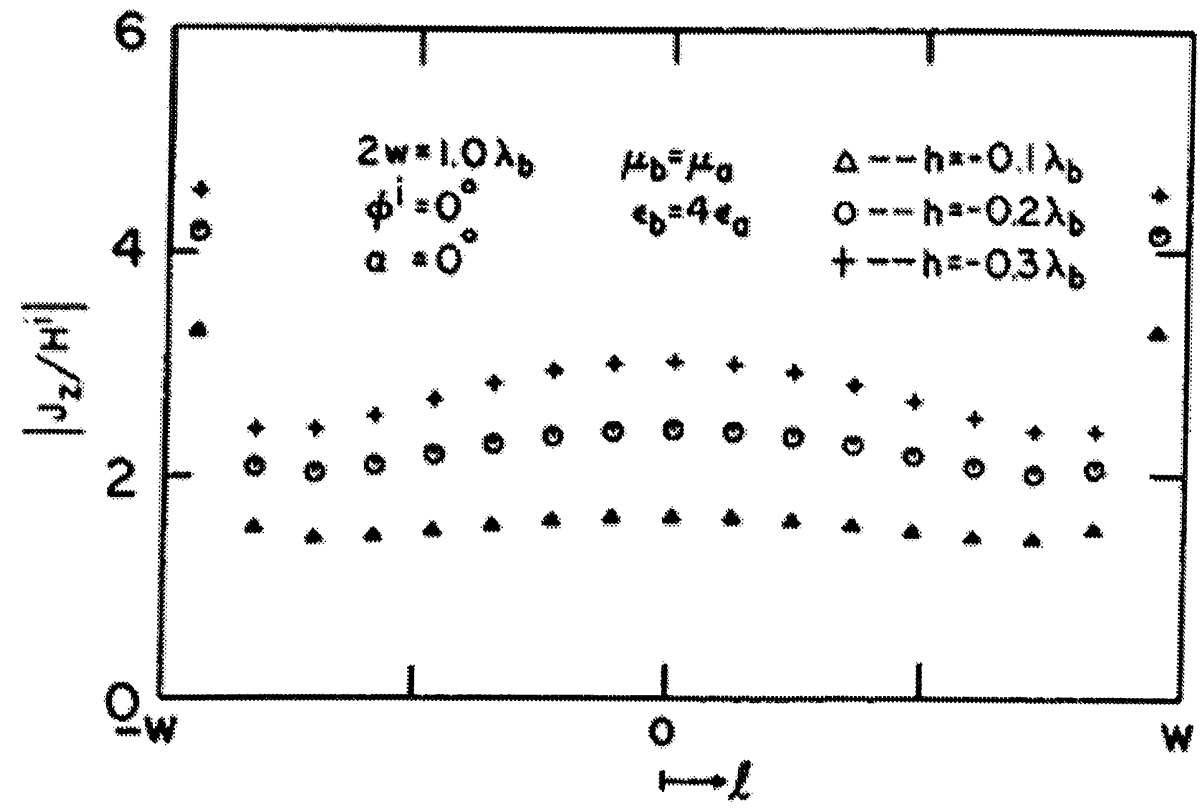

Figure 4.4: Normalized current density on a strip below interlace for $\theta=0^{\circ}$ and the TM excitation (reproduced from [16]). See Fig. 4.1 and the following text for the geometry and its parameters.

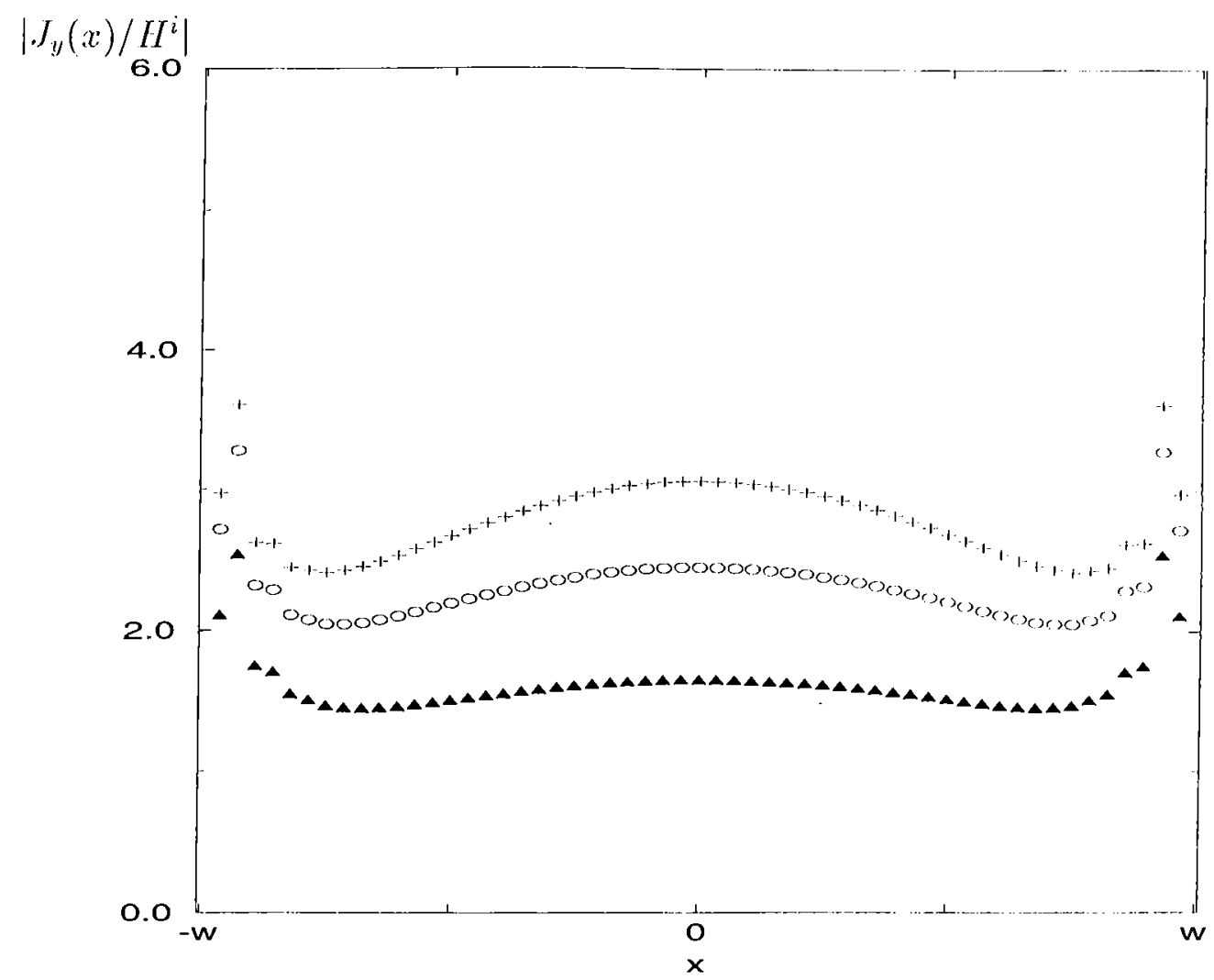

ligure 1.5: Normalized current density on a strip below interface for $\theta=0^{\circ}$ and the TM excitation from the application of the spatial-domain MoM. Seo Fig. 1.1 and the following text for the geometry and its parameters. 


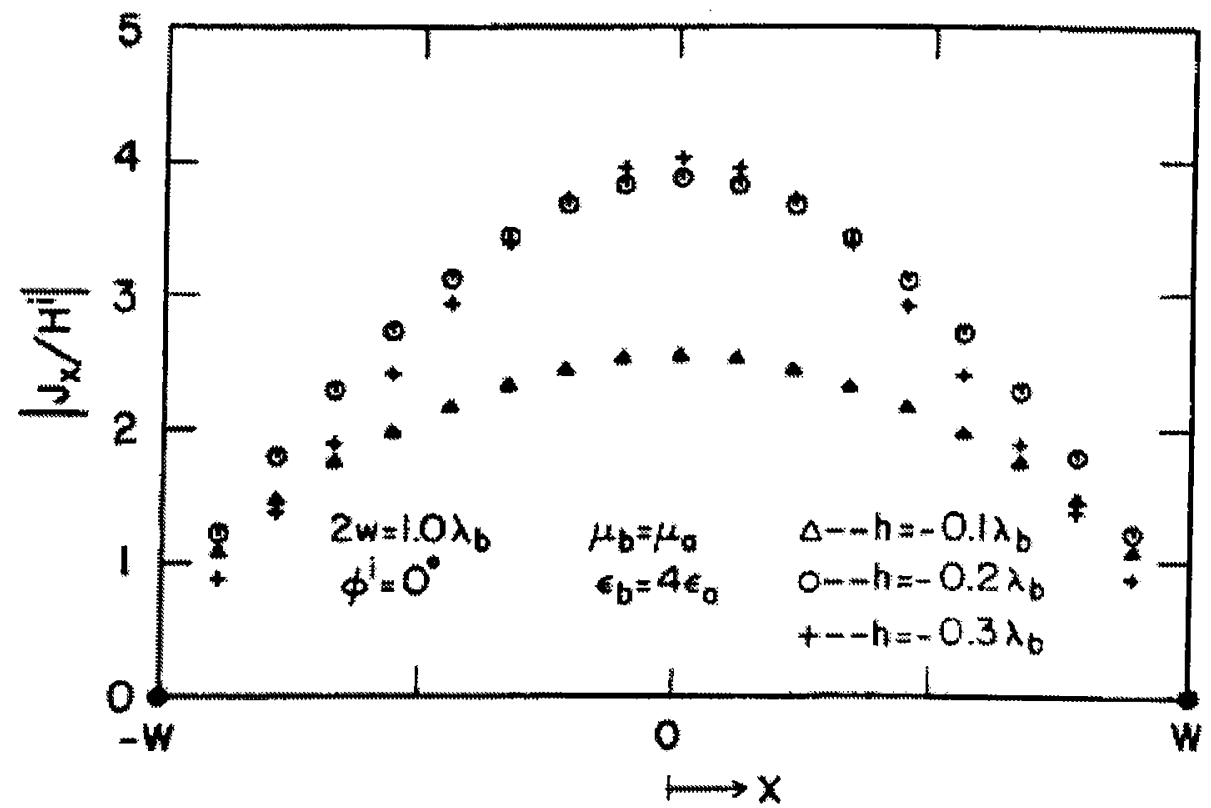

Figure 4.6: Normalized current density on a strip below interface for $\theta=0^{\circ}$ and the TE excitation (reproduced from [17]). See Fig. 4.1 and the following text for the geometry and its parameters.

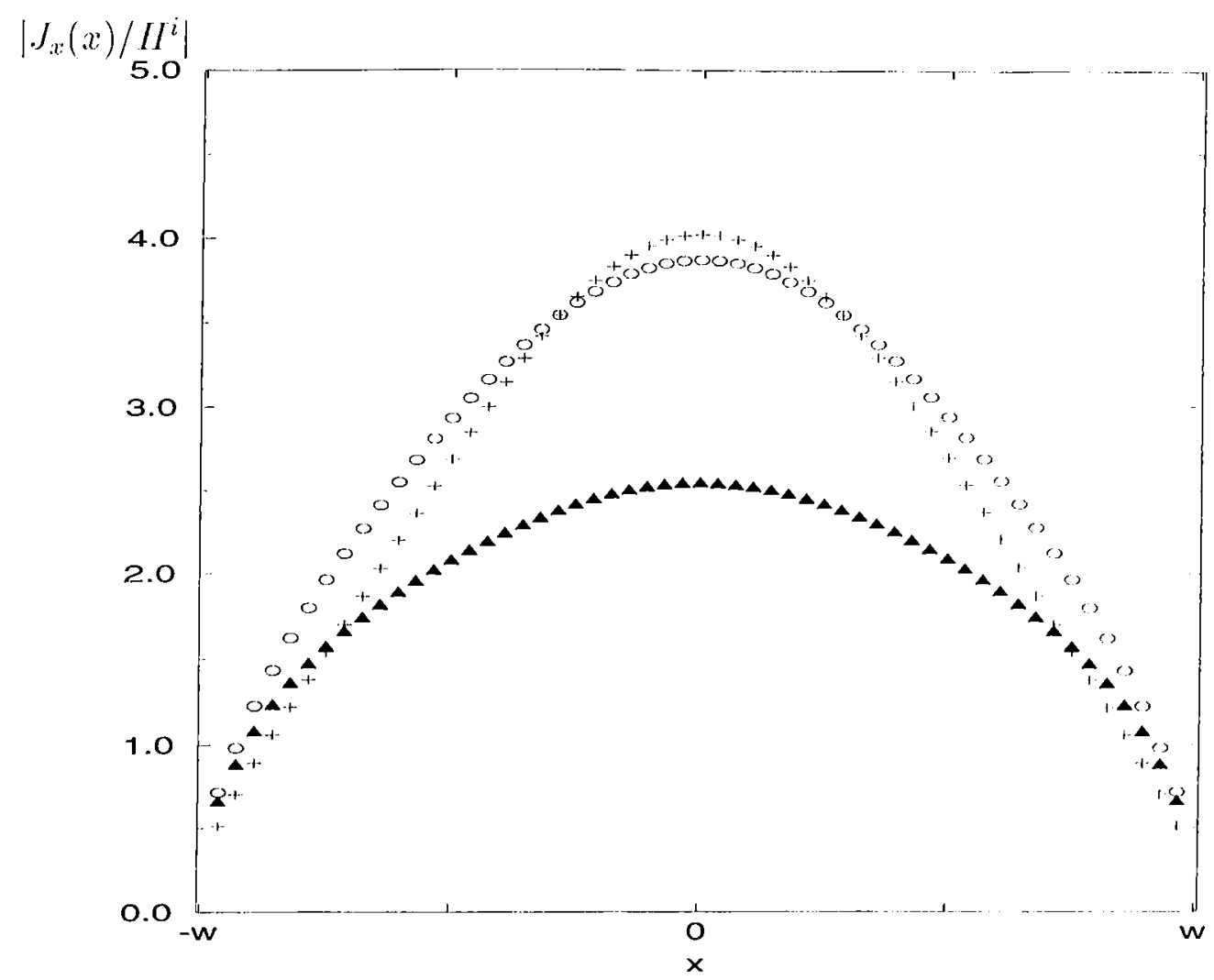

ligure 1.7: Normalized current density on a strip below interface for $0=0^{\circ}$ and the TE excitation from the application of the spatial-clomain MoM. See rig. 1.1 and the following text for the geometry and its parameters. 


\subsubsection{Analytical Solution of Cylinders in a Homogeneous Medium}

\section{Incident}

wave

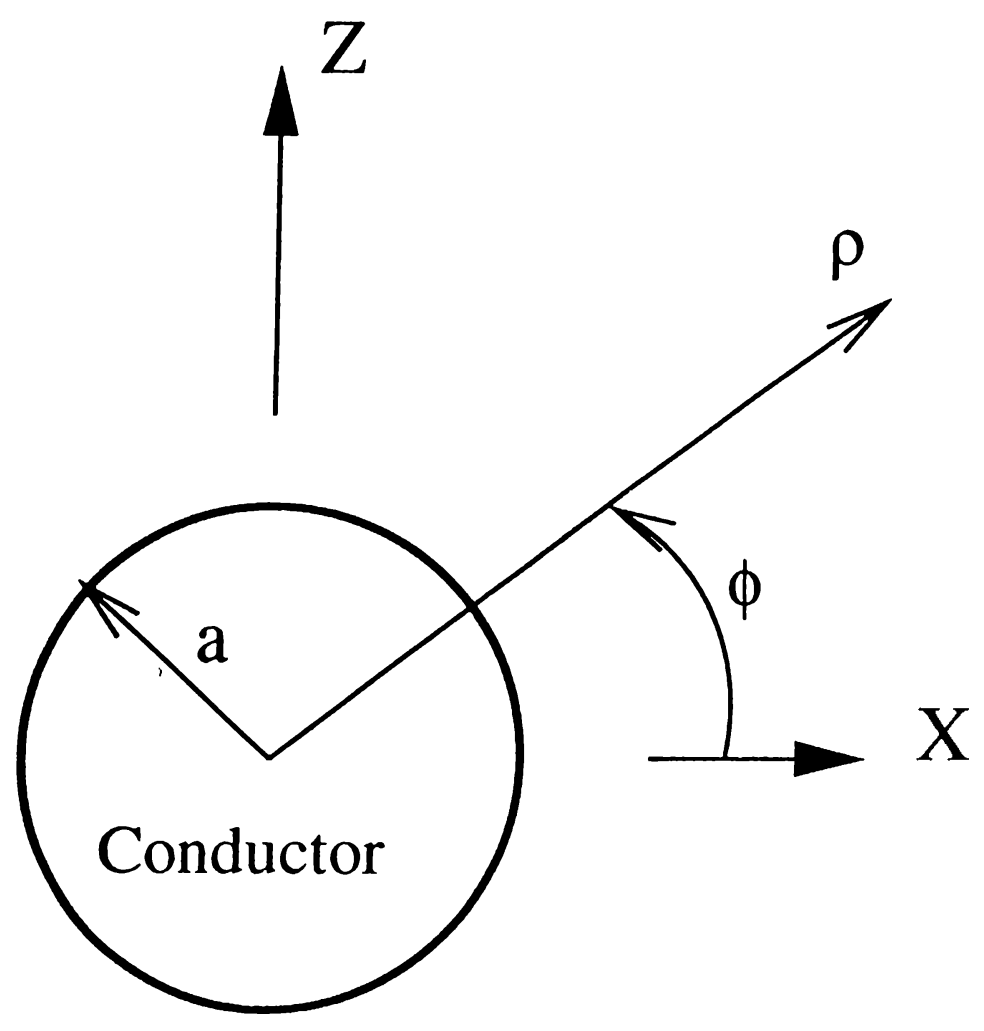

Figure 4.8: A plane wave incident upon a conducting cylinder in a homogeneous medium

The current density on a conducting cylinder in a homogeneous medium is calculated in this section for a plane wave incidence. For the T.M excitation. the incident field is $y$-polarized and written as

$$
E_{y}^{i}=E_{0} \epsilon^{-j k x}=E_{0} \epsilon^{-j k \rho \cos \phi}
$$

It is often convenient to express the elementary wave functions of one coordinate system in terms of those of another coordinate system. Two coordinate systems are considered to be distinct if their origins or orientations are different. even 
though they may be geometrically the same. This type of expressions are referred to as wave transformations [18]. Using the wave transformation for Eq. (4.1), the incident field can be written as

$$
E_{y}^{i}=E_{0} \sum_{n=-\infty}^{\infty} j^{-n} J_{n}(k \rho) \epsilon^{j n \phi}
$$

The total field with the conducting cylinder present is the sum of the incident and scattered fields, that is,

$$
E_{y}=E_{y}^{i}+E_{y}^{s}
$$

To represent outward-traveling waves, the scattered field must be of the form

$$
E_{y}^{s}=E_{0} \sum_{n_{i}=-\infty}^{\infty} j^{-n} a_{n} H_{n}^{(2)}(k \rho) \epsilon^{j n \phi}
$$

hence the total field is

$$
E_{y}=E_{0} \sum_{n=-\infty}^{\infty} j^{-n}\left[J_{n}(k \rho)+a_{n} H_{n}^{(2)}(k \rho)\right] \epsilon^{j n \phi}
$$

At the cylinder. the boundary condition $E_{y}=0$ at $\rho=a$ must be met. It is evident from Eq. (4.5) that this condition is satisfied if

$$
a_{n}=\frac{-J_{n}(k a)}{H_{n}^{(2)}(k \cdot a)}
$$

which completes the solution. The surface current density on the cylinder may. be obtained from

$$
J_{y}=\left.H_{0}\right|_{\rho=a}=\left.\frac{1}{j \omega \mu} \frac{\partial E_{y}}{\partial \rho}\right|_{\rho=a}
$$

['sing Eqs. (4.4) and (4.5). the current density for T.M excitation can be written as

$$
J_{y}=\frac{-2 E_{0}}{\omega \cdot \mu \pi a} \sum_{n=-\infty}^{\infty} \frac{j^{-n} e^{j n a}}{H_{n}^{(2)}(k \cdot a)}
$$


For the TE excitation case. the incident field is polarized transverse to $y$. so the magnetic field in $y$ direction can be written as

$$
H_{y}^{i}=H_{0} \epsilon^{-j k r}=H_{0} \sum_{n=-\infty}^{\infty} j^{-n} J_{\pi}(k \rho) \epsilon^{j n \phi}
$$

and the total field is written as

$$
H_{y}=H_{y}^{i}+H_{y}^{s}
$$

To represent outward-traveling waves, the scattered field is of the form

$$
H_{y}^{s}=H_{0} \sum_{n=-\infty}^{\infty} j^{-n} b_{n} H_{n}^{(2)}(k \rho) \epsilon^{j n o}
$$

and the total field is given by

$$
H_{y}=H_{0} \sum_{n=-\infty}^{\infty} j^{-n}\left[J_{n}(k \rho)+b_{n} H_{n}^{(2)}(k \cdot \rho)\right] e^{j n \phi}
$$

In this case, the boundary condition becomes $E_{0}=0$ at $\rho=a$. From the field equations,

$$
\begin{aligned}
E_{0} & =\frac{1}{j \omega \epsilon}\left(\Gamma \times \hat{a}_{y} H_{y}\right)_{0} \\
& =\frac{j k}{i \epsilon} H_{0} \sum_{n=-\infty}^{\infty} j^{-n}\left[J_{n}^{\prime}+b_{n} H_{n}^{(2) \prime}(k \rho)\right] \epsilon^{j n \sigma}
\end{aligned}
$$

and the boundary condition is satisfied if

$$
b_{r_{i}}=\frac{-J_{n}^{\prime}(k \cdot a)}{H_{n}^{(2) \prime}(k \cdot a)}
$$

Then the surface current density on the cylinder becomes

$$
J_{\phi}=\left.H_{y}\right|_{r=2}=\frac{j 2 H_{0}}{\pi k a} \sum_{n=-\infty}^{\infty} \frac{j^{-\pi} \epsilon^{j n \phi}}{H_{n}^{(2) \prime}(k a)}
$$

for the TE excitation case. 
The analytical solution of current densities of the cylinder in homogeneous medium obtained for the TM and TE excitations has been compared with those obtained using using the spatial-domain MOM formulation. Figs. 4.9 and 4.10 show the results of the analytical and numerical methods for both the TM and TE excitations, respectively: In this example, the radius of the cylinder is chosen as $k a=1$ and 5 , the angle of incidence, as shown in Fig. 4.8 , is $-90^{\circ}$. It is observed that the numerical results agree quite well with the analytical results. 


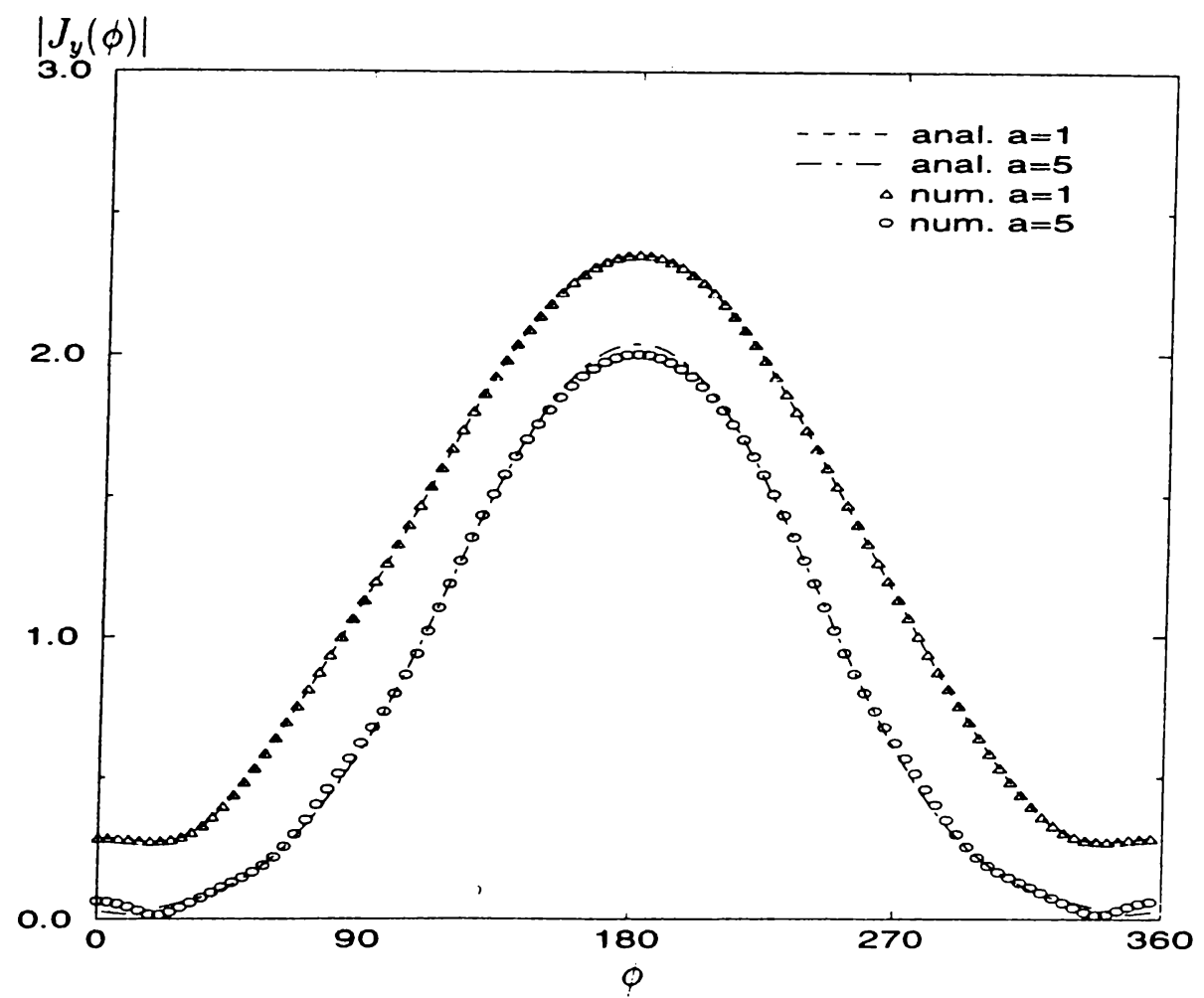

Figure 4.9: The magnitude of the current density on a cylinder in a homogeneous medium for TM excitation

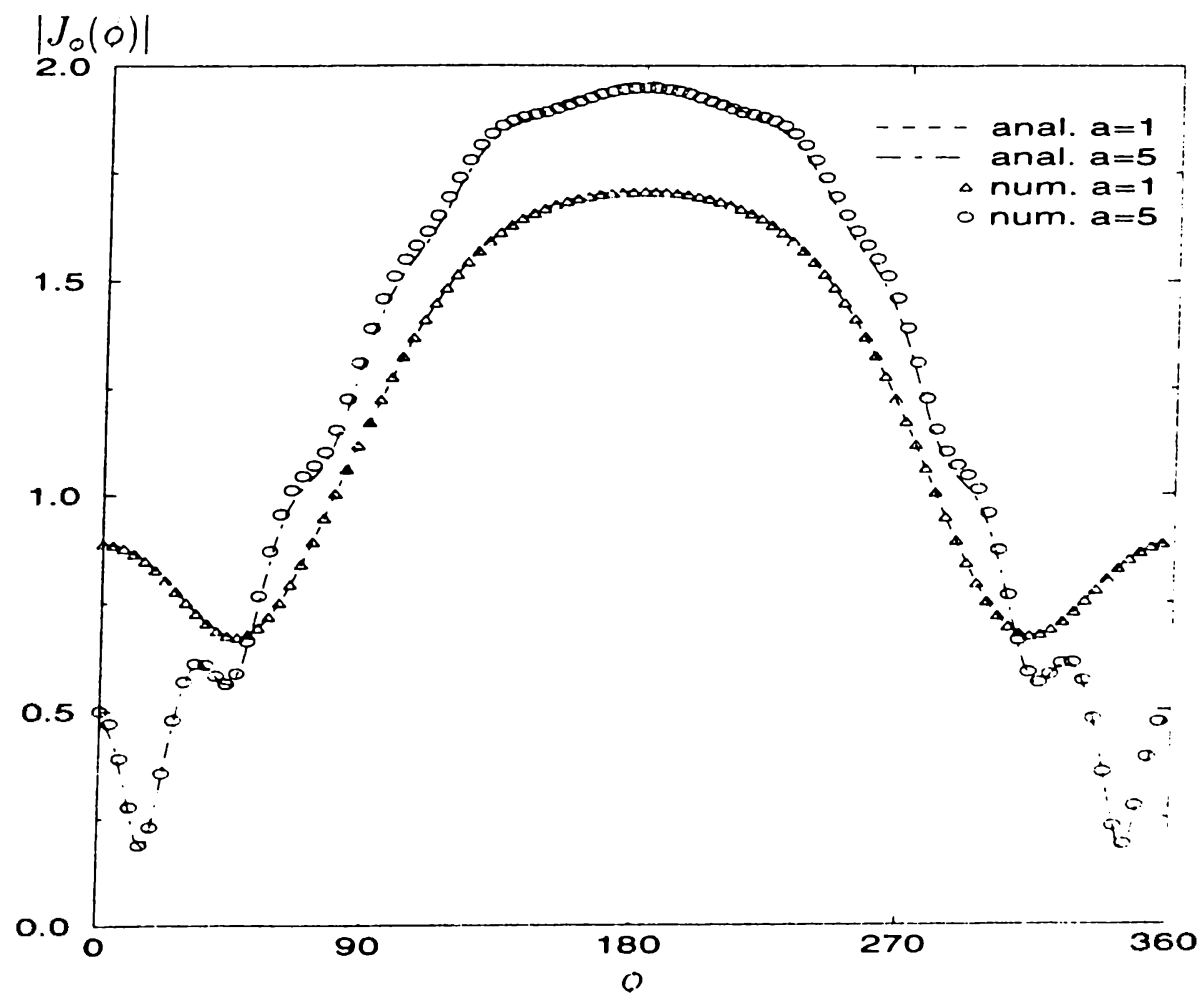

Figure t.10: The magnitude of the current density on a cylinder in a homogeneous medium for TE excitation 


\subsubsection{A Conducting Cylinder in an Inhomogeneous Medium}

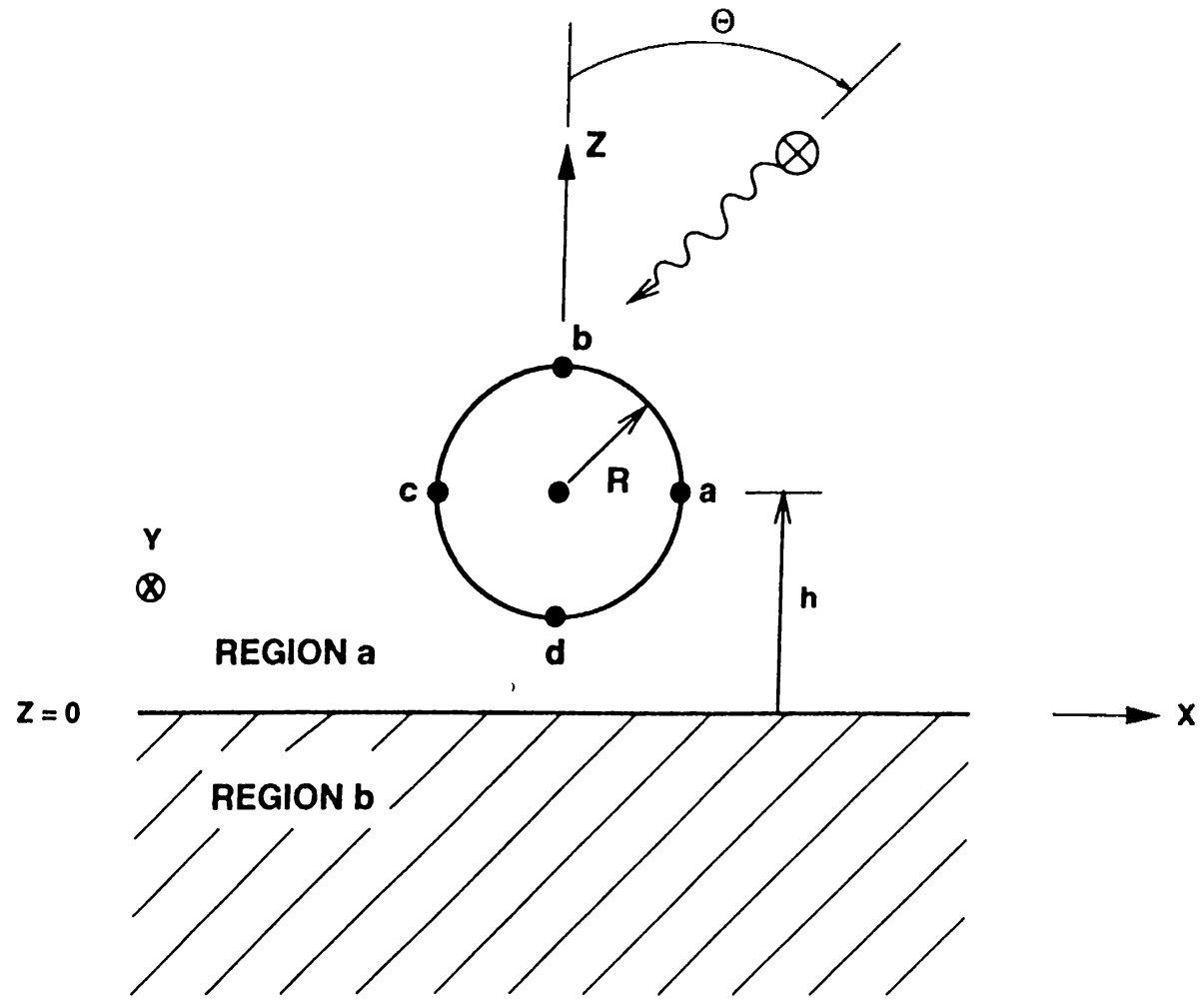

Figure 4.11: Conducting cylinder located near interface between two semi-infinite half-spaces and illuminated by a plane wave in upper half-space.

After having examined a conducting cylinder in a homogeneous medium with a view of ralidating the code. the spatial-domain MoM, in this section the example of a conducting cylinder located near interface between two semi-infinite halfspaces is studied for the T.M excitation, Fig. 4.11. The parameters used in this study are as follows: $\epsilon_{r_{a}}=1, \epsilon_{r_{b}}=4$; the incident angle $\theta=0^{\circ}$ : the radius of the cylinder $R=0.375 \lambda_{2}: h=R+0.05 \lambda_{a}$.

For the conducting geometries having both $x$ and z rariations. such as tilted surfaces. both of the Green s functions, $G_{x}^{q_{e}}$ and $C_{i}=$ are required and they are not equal to each other except for the case of a homogeneous medium. For these 


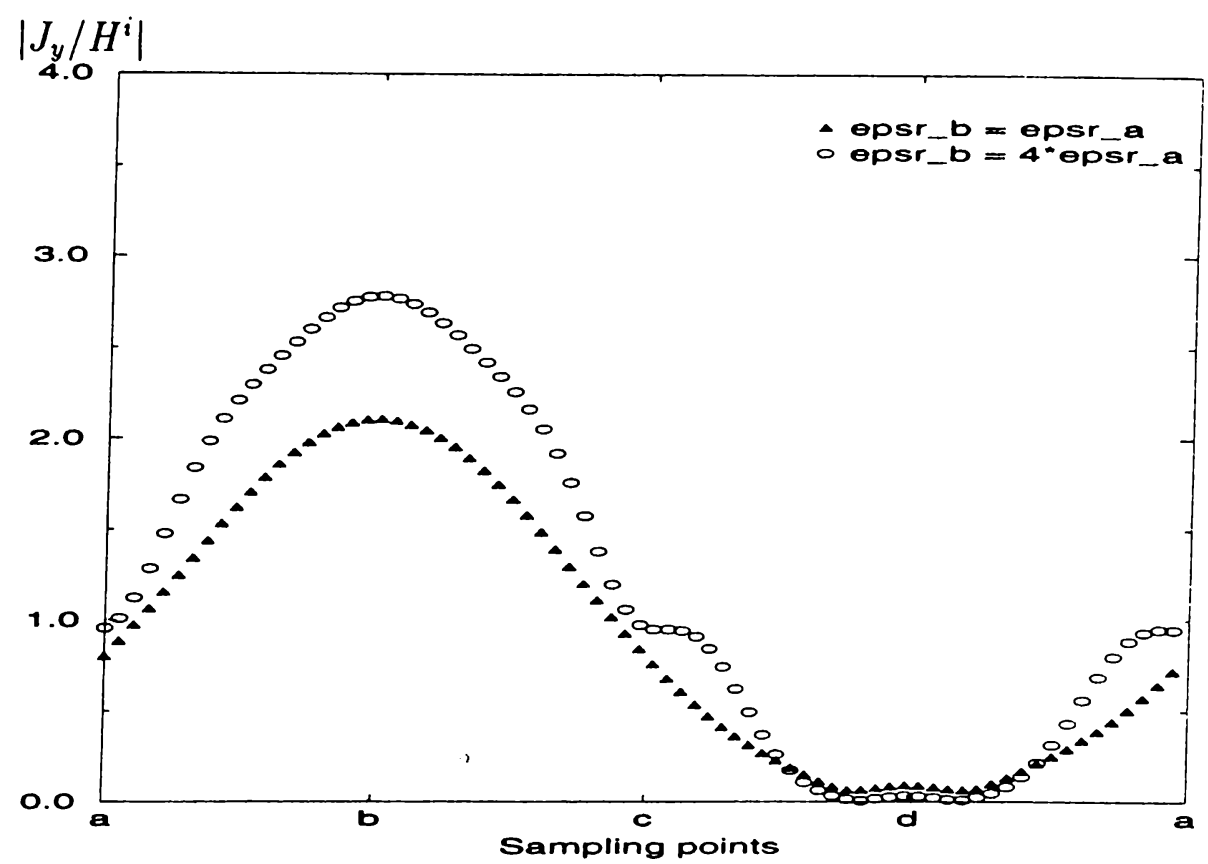

Figure 4.12: Normalized current density on a circular cylinder of radius $R=$ $0.375 \lambda_{a}$ for the TM excitation with various sampling points along the circumferential direction. See Fig. 4.11 and the following text for the geometry and its parameters.

geometries, the evaluation of the integral equation of the integral equation is not possible for the TE excitation since both $G_{x}^{q_{e}}$ and $G_{z}^{q_{e}}$ are required for the formulation. Therefore only the TM excitation is considered in the example of conducting cylinder in this section since the geometry includes both $r$ and $z$ variations.

The normalized current densities for homogeneous case $\left(\epsilon_{r_{b}}=\epsilon_{r_{a}}\right)$ and for inhomogeneous case $\left(\epsilon_{r_{b}}=4 \epsilon_{r_{a}}\right)$ are calculated and given in Fig. 4.12 for a rarious sampling points along the circumferential direction. The results obtained here agree with the results in the literature. [16]. 


\subsubsection{A Rectangular Cylinder in an Inhomogeneous Medium}

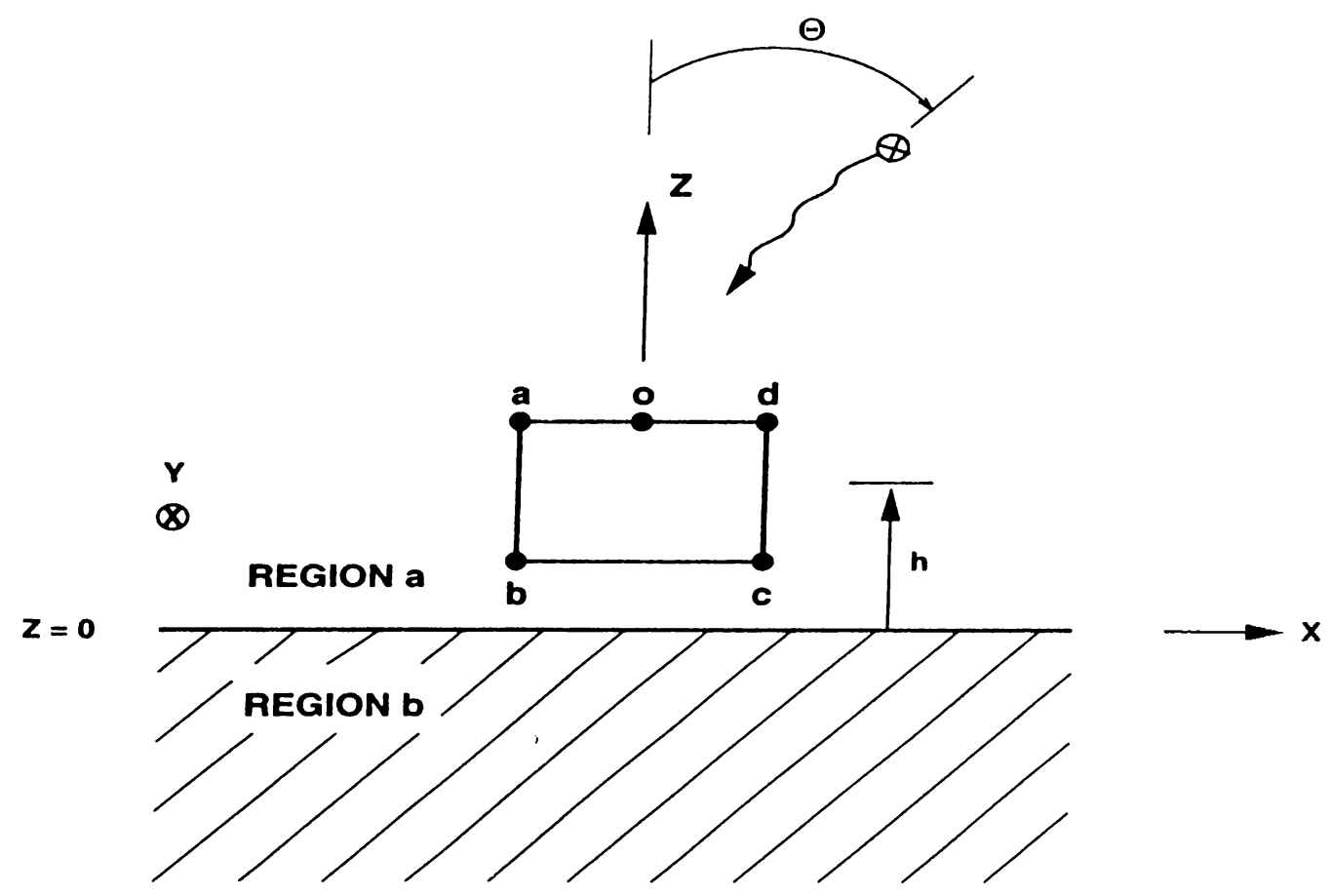

Figure 4.13: Rectangular cylinder located near interface between two semi-infinite half-spaces and illuminated by a plane wave in upper half-space

In this example. a rectangular conducting cylinder located near the interface between two semi-infinite half-spaces is examined for the TM and TE excitations. Fig. 4.13. Since there is no tilted surface for this geometry it is possible to find the current density for the TE excitation as well. The geometry is defined as follows: $\epsilon_{r_{a}}=1 . \epsilon_{r_{b}}=4$ : the incidence angle $\theta=0^{\circ}$. width $=0.25 \lambda_{a}$. height $=0.1 \lambda_{a} ; h=0.05 \lambda_{a}$. The normalized current densities for homogeneous case $\left(\epsilon_{r_{b}}=\epsilon_{r_{a}}\right)$ and for inhomogeneous case $\left(\epsilon_{r_{b}}=4 \epsilon_{r_{a}}\right)$ are calculated for both the TM and TE excitations. as given in Figs. 4.14 and 4.15. respectively: and it is observed that the results obtained here perfectly agree with the results found in the literature. [16], [1i]. 


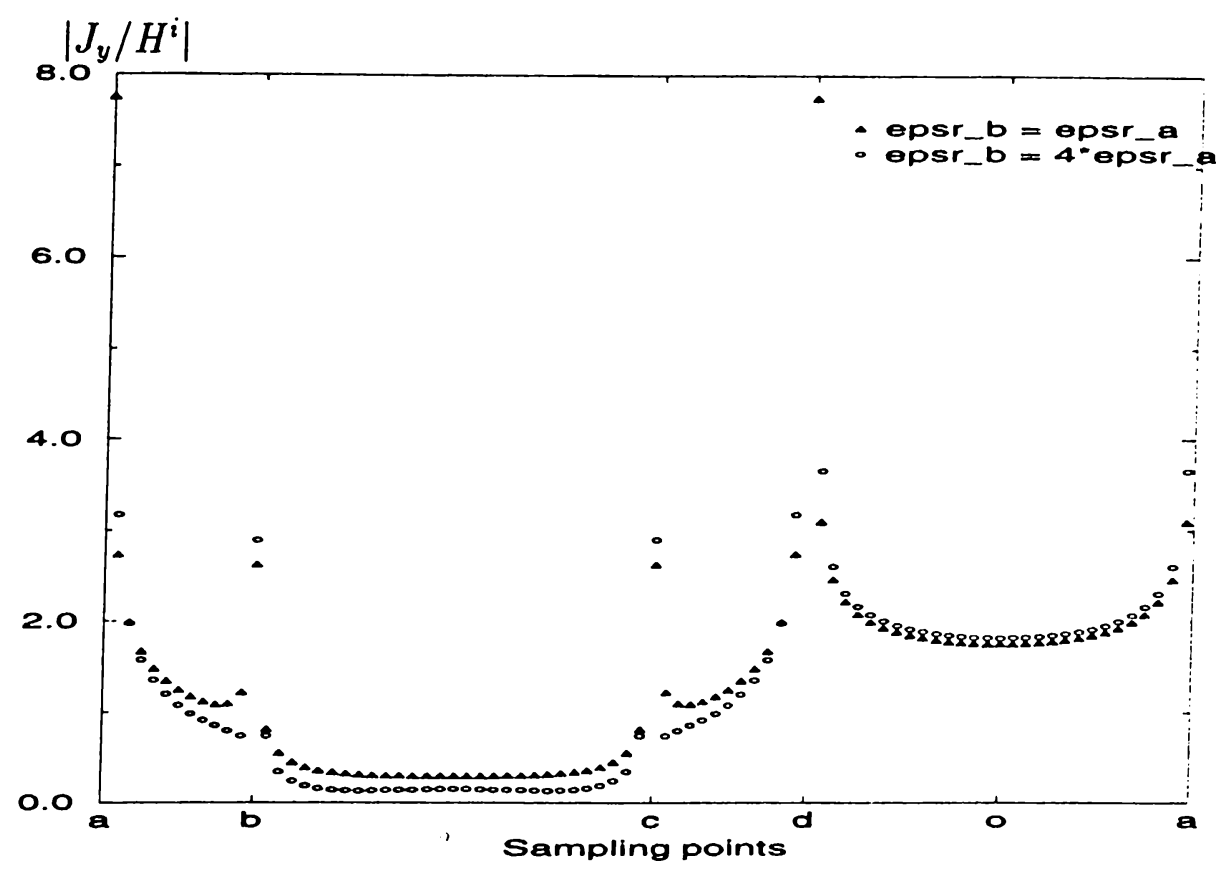

Figure 4.14: Normalized current density on a rectangular cylinder of cross section $0.25 \lambda_{a}$ (width) and $0.1 \lambda_{a}$ (height) for the TM excitation with various sampling points along the periphery: See Fig. 4.13 and the following text for the geometry and its parameters.

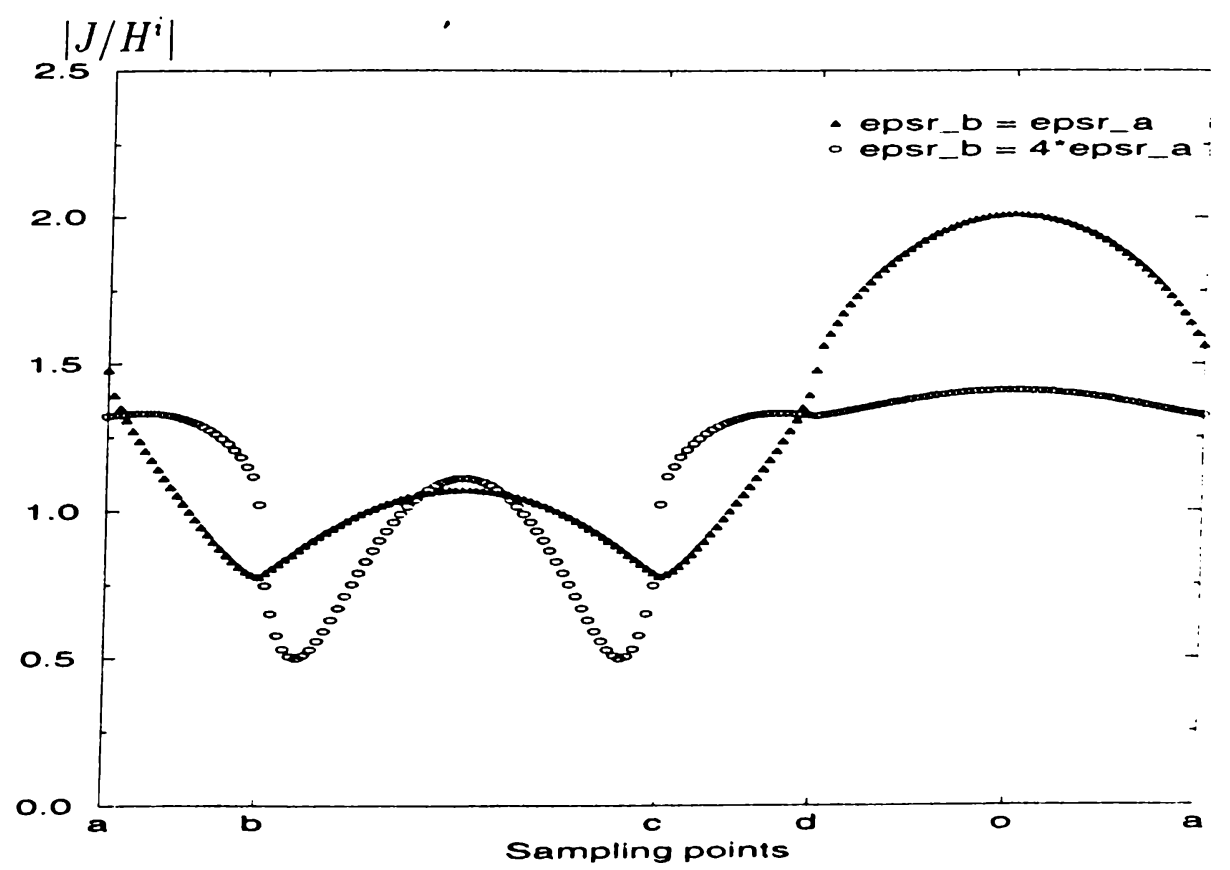

Figure 4.15: Normalized current density on a rectangular cylinder of cross section $0.25 \lambda_{a}$ (width) and $0.1 \lambda_{a}$ (height) for the TE excitation with rarious sampling points along the periphery: See Fig. 4.13 and the following text for the geometry and its parameters. 


\subsection{Comparison of the Methods}

So far, only the spatial-domain MoM has been applied to some geometries. such as strips, conducting cylinders and conducting rectangular cylinders, and the results have been compared to those available in the literature. Since the other techniques introduced in Chapter 3, namely the spectral-domain and the FFT approaches, have been specifically developed for planar geometries, they can not be employed in the analysis of non-planar geometries such as the examples of cylinders. In this section, these three techniques are applied to several geometries in multilayer media, and their performances are compared.

For the first example, a strip of width $2 w=4 \lambda_{a}$ at the interface of two semiinfinite layers with the dielectric constants of $\epsilon_{r_{a}}=1$ and $\epsilon_{r_{b}}=4$ is analyzed by using the three approaches mentioned above, Fig. 4.1. The number of basis functions is chosen to be $10 \tau$ for the TE case and 109 for the TM case. The normalized current densities obtained from these three approaches are given in Figs. 4.16 and 4.17 for the TE excitation and in Figs. 4.18 and 4.19 for the TM excitation. As it is observed, the results are almost indistinguishable, so the numerical efficiency of these approaches can be assessed without any bias. Table 5.1 shows the CPU times of the spectral and the special domain approaches and the FFT approach for the TE and TM excitations. It should be noted that the spectral domain formulation is much more efficient than the other two approaches. 


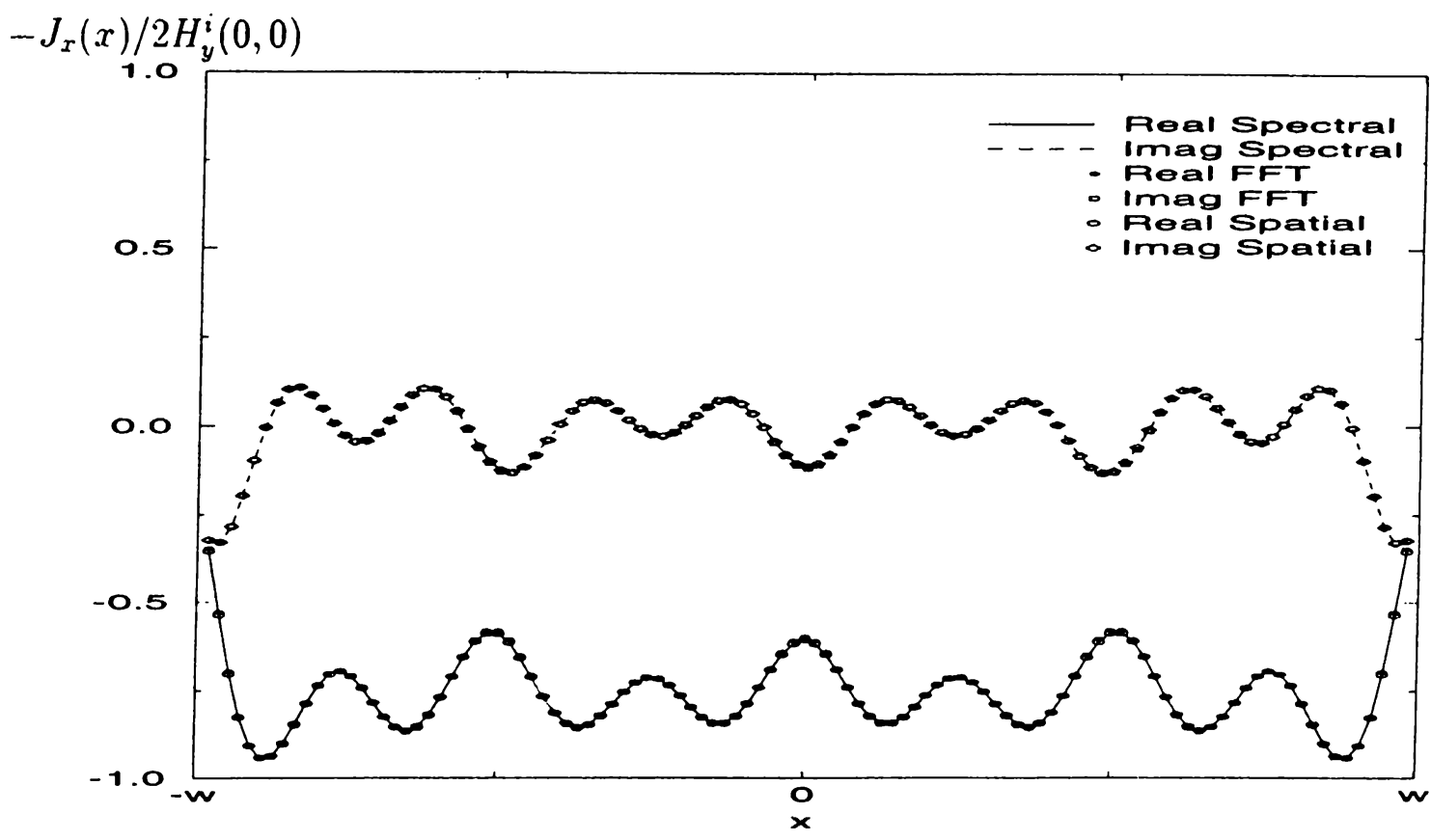

Figure 4.16: The real and imaginary parts of the normalized current densities for the TE excitation and for $\theta=0^{\circ}$

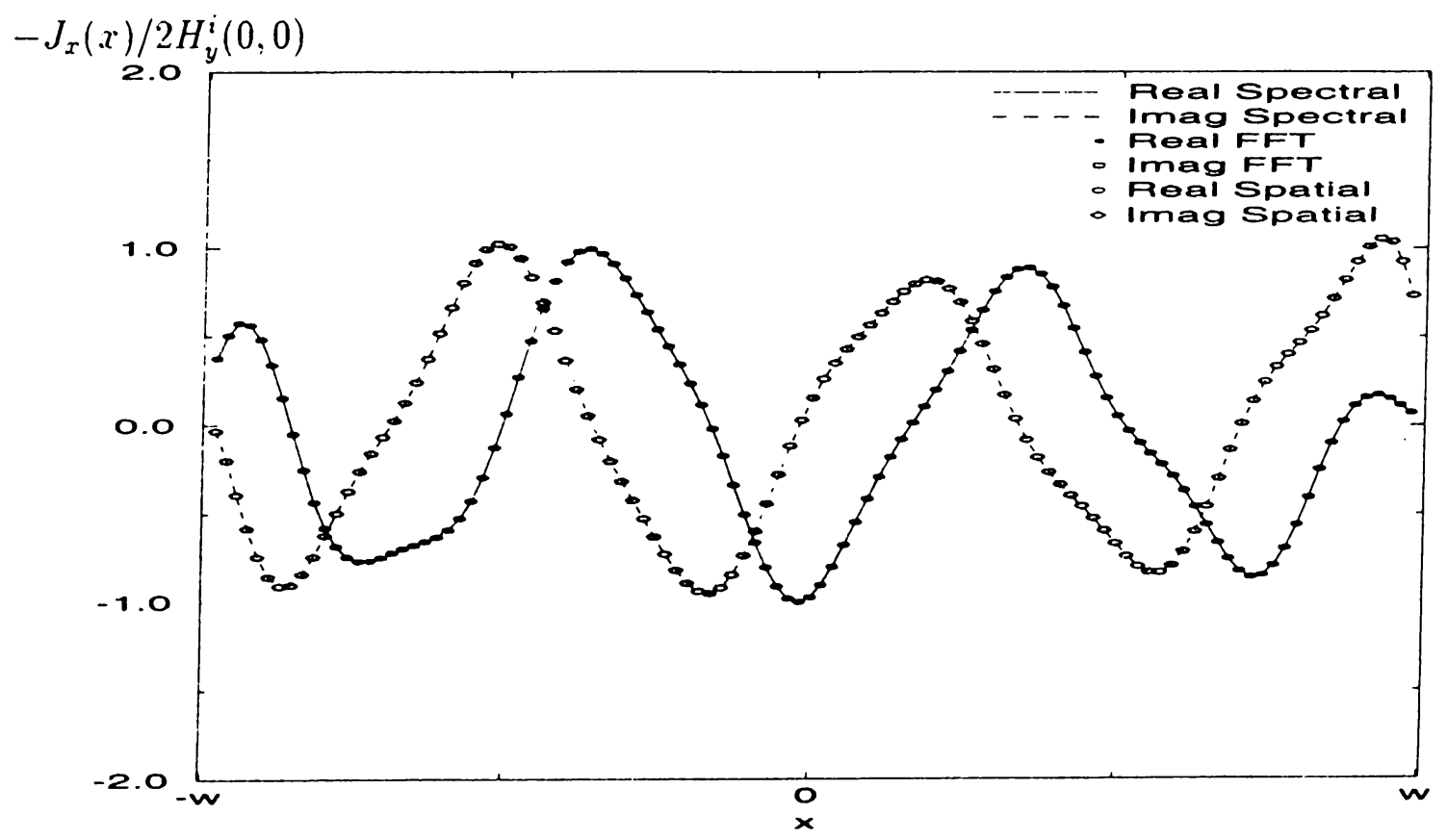

Figure 4.17: The real and imaginary parts of the normalized current densities for the ' $T$ E excitation and for $\theta=-45^{\circ}$ 


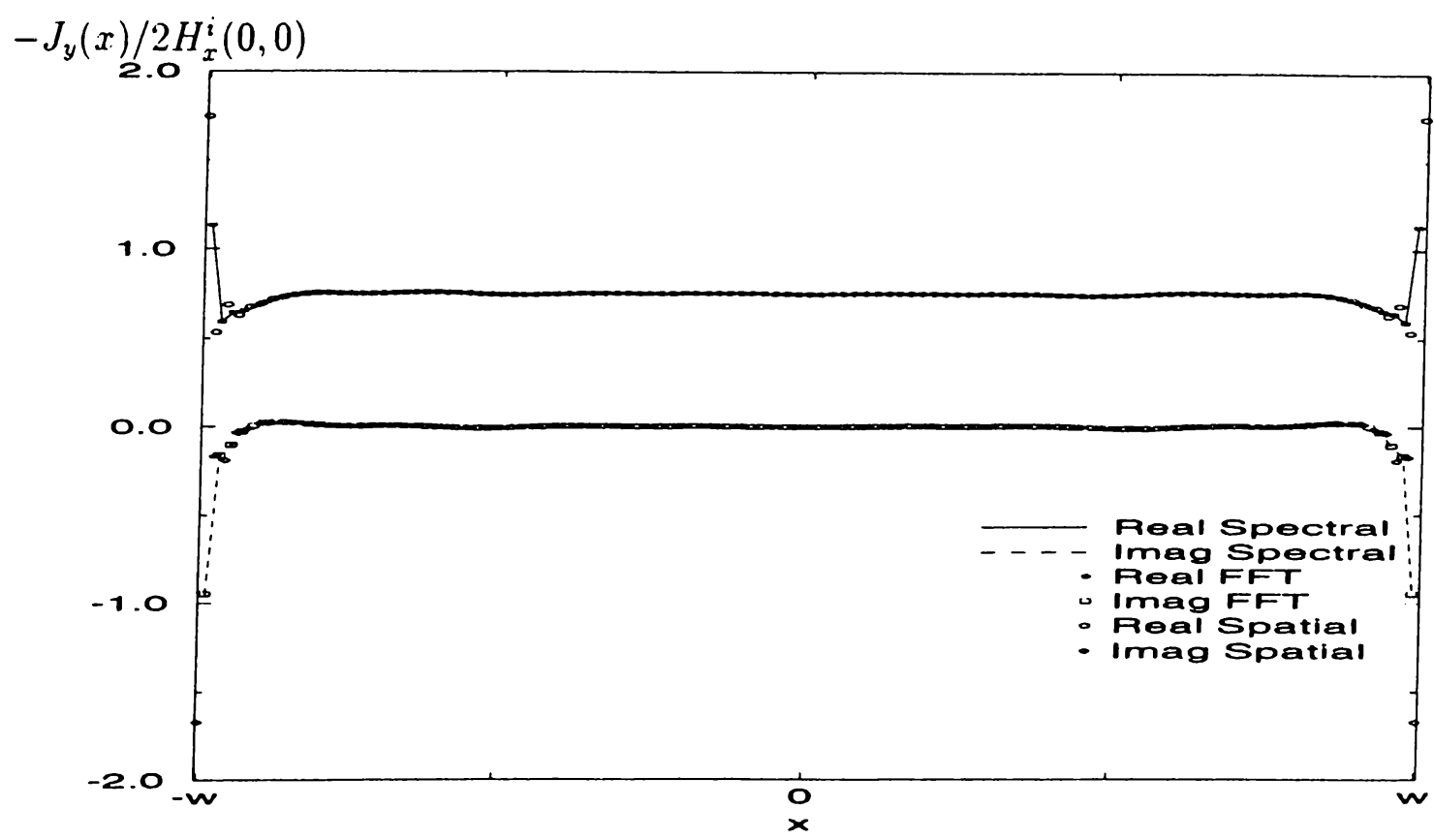

Figure 4.18: The real and imaginary parts of the normalized current densities for the TM excitation and for $\theta=0^{\circ}$

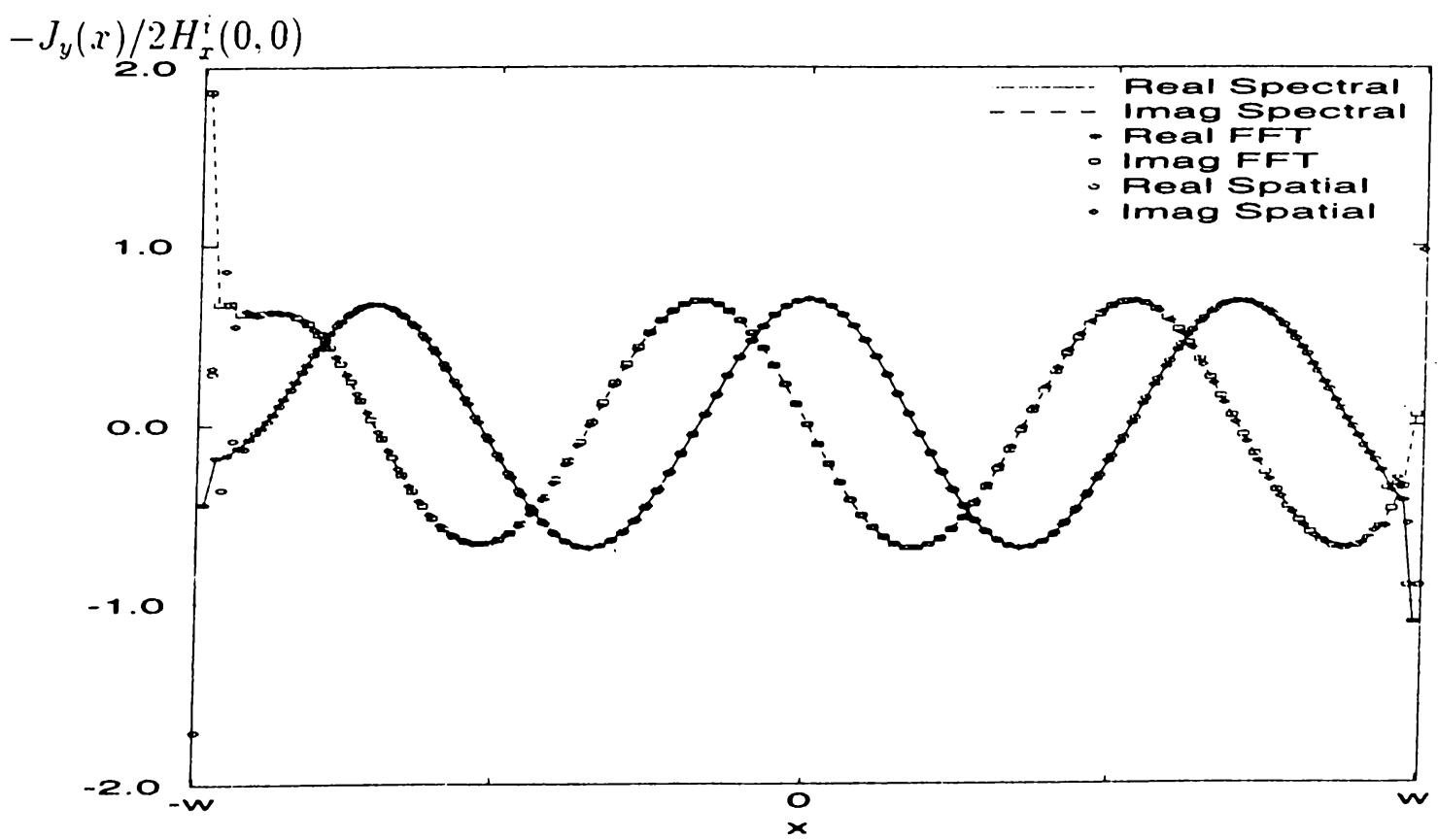

Figure 4.19: The real and imaginary parts of the normalized current densities for the TM excitation and for $A=-45^{\circ}$ 


\begin{tabular}{||c|c|c|c|c||}
\hline \multirow{2}{*}{} & \multicolumn{4}{|c||}{ CPU time (s) } \\
\cline { 2 - 5 } & \multicolumn{2}{|c|}{ TE } & \multicolumn{2}{c||}{ TM } \\
\cline { 2 - 5 } & $\theta=0^{\circ}$ & $\theta=-45^{\circ}$ & $\theta=0^{\circ}$ & $\theta=-4.5^{\circ}$ \\
\hline Spectral & 13.7 & 16.8 & 10.1 & 9.8 \\
\hline FFT & 16.3 & 33.0 & 14.9 & 15.0 \\
\hline Spatial & 361.7 & 416.5 & 107.5 & 108.2 \\
\hline
\end{tabular}

Table 4.1: The CPU times of the spectral domain, the spatial domain and the FFT approaches for TE and TM excitations

The geometry of the next example is given in Fig. 4.1. For this example, the geometry is defined as follows: $\epsilon_{r_{b}}=4$ and $\epsilon_{r_{a}}=1$ : the width of the strip, $2 w=\lambda_{b} ; h=-0.1 \lambda_{b},-0.2 \lambda_{b}$ and $-0.3 \lambda_{b}$, meaning that the strip is in the lower layer; and the angle of incidence, $\theta=0^{\circ}$. The number of the basis functions is 55 for the TE case and 57 for the TM case. Table 4.2 shows the C.PL times of the spectral and the spatial domain approaches and the FFT approach for the TE and T.M excitations. Again. the spectral domain approach uses the less computation time as compared to the other approaches. The normalized current densities obtained from these three approaches are given in Figs. 4.20 and 4.21 for the TE and TM excitations, respectively, for $h=-0.1 \lambda_{b}$. 


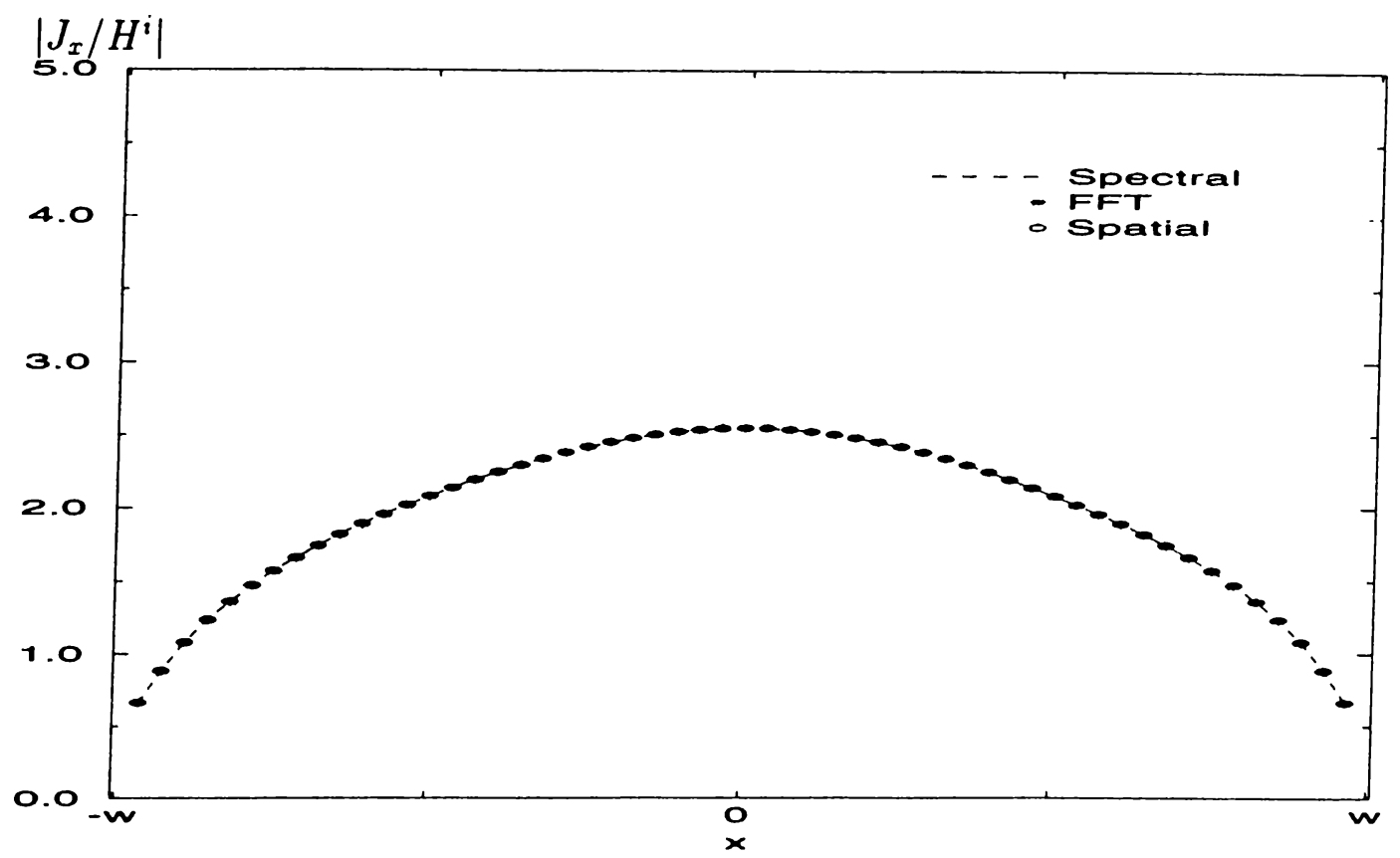

Figure 4.20: Normalized current densities on the strip for the TE excitation and $h=-0.1 \lambda_{b}$

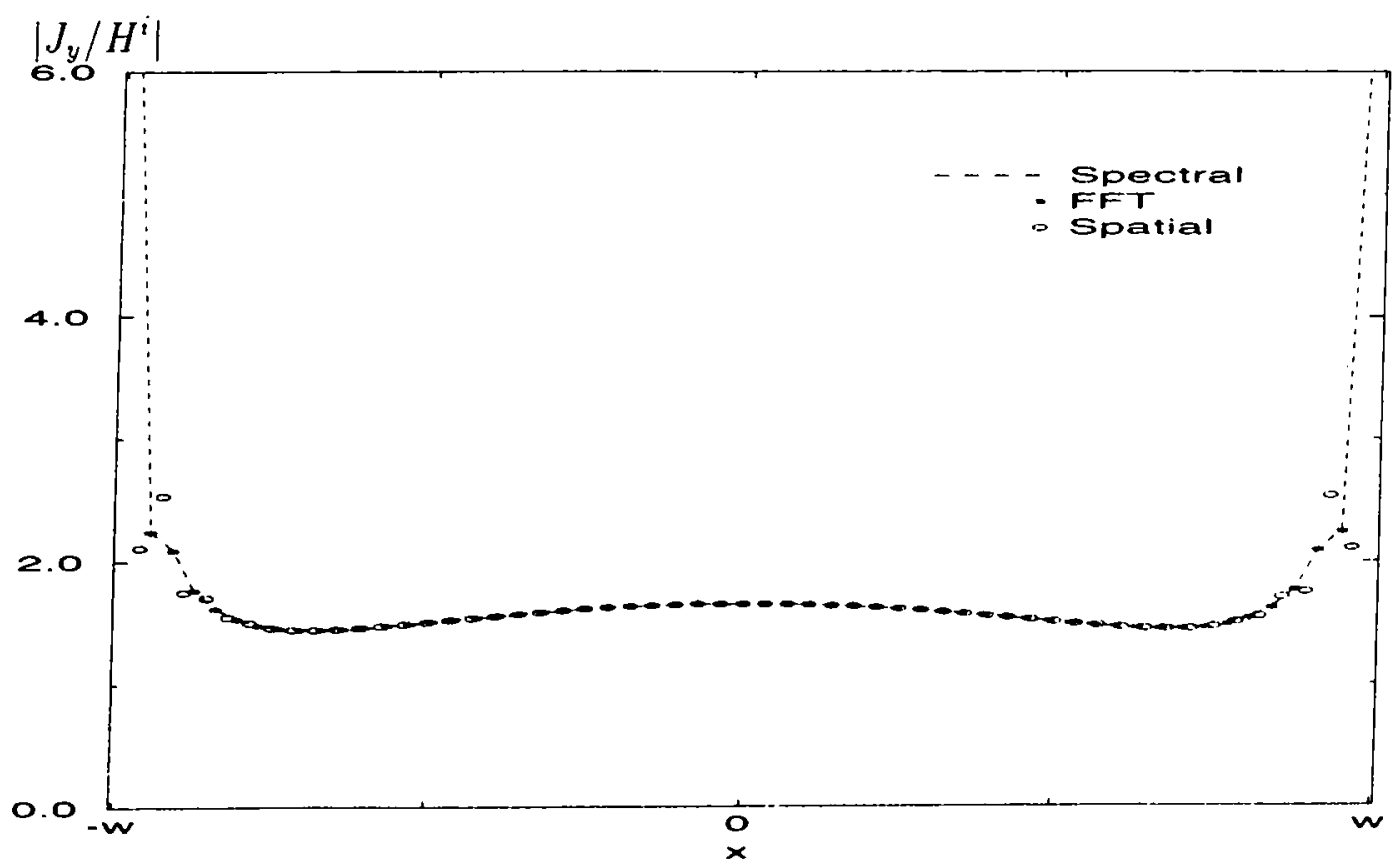

Figure 4.21: Yormalized current densities on the strip for the T.II excitation and $h=-0.1 \lambda_{b}$ 


\begin{tabular}{||c|c|c|c||}
\hline \multirow{2}{*}{} & \multicolumn{3}{|c||}{ CPU time (s) } \\
\cline { 2 - 4 } & $h=-0.1 \lambda_{0}$ & $h=-0.2 \lambda_{0}$ & $h=-0.3 \lambda_{0}$ \\
\hline Spectral & 10.5 & 9.7 & 9.7 \\
\hline FFT & 26.5 & 54.9 & 53.7 \\
\hline Spatial & 125.5 & 124.6 & 124.1 \\
\hline
\end{tabular}

(a)

\begin{tabular}{||c|c|c|c||}
\hline \multirow{2}{*}{} & \multicolumn{3}{|c||}{ CPU time (s) } \\
\cline { 2 - 4 } & $h=-0.1 \lambda_{0}$ & $h=-0.2 \lambda_{0}$ & $h=-0.3 \lambda_{0}$ \\
\hline Spectral & 11.4 & 11.2 & 11.6 \\
\hline FFT & 25.0 & 24.4 & 24.4 \\
\hline Spatial & 39.6 & 39.3 & 39.4 \\
\hline
\end{tabular}

(b)

Table 4.2: The CPU times of the spectral domain, the spatial domain and the FFT approaches for (a) TE and (b) TM excitations

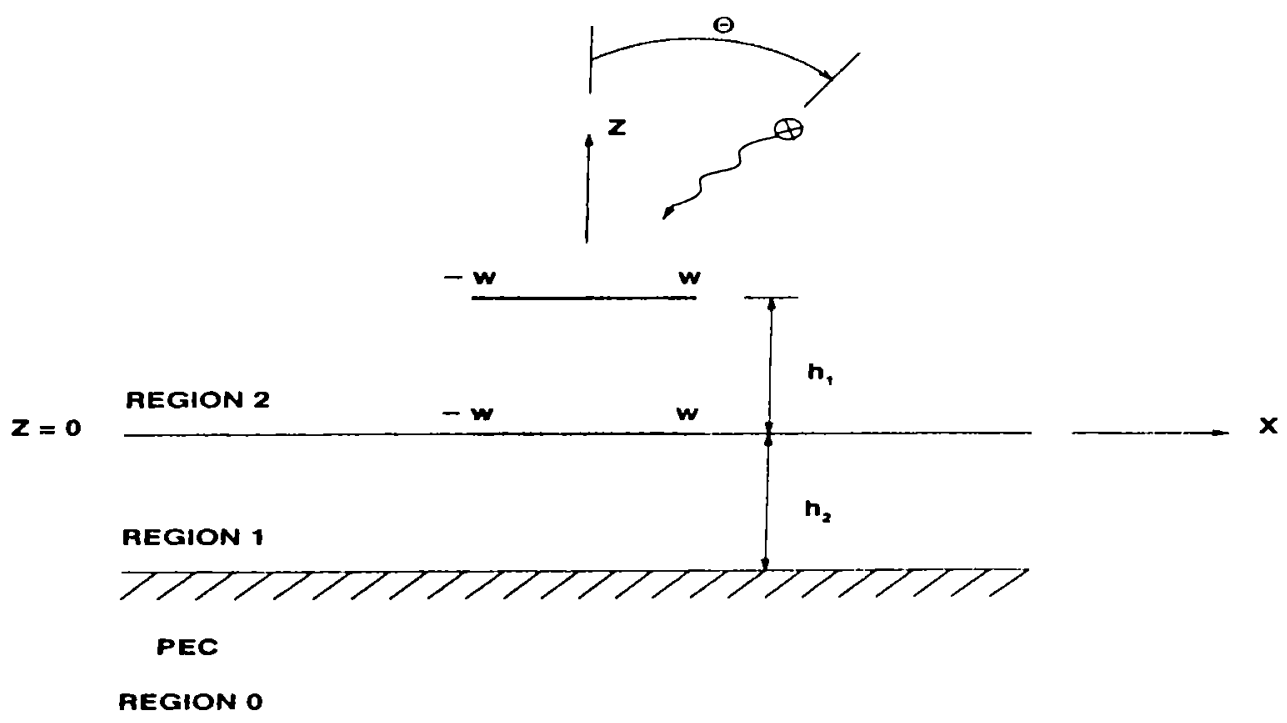

Figure 4.22: A two-strip, three layer geometry:

The geometry of the next example is given in Fig. 4.22. where Region 0 is PEC. $\epsilon_{r_{1}}=4$ and $\epsilon_{r_{2}}=1$. the width of the strips $2 u=0.5 \lambda_{2}$. and $h_{1}=h_{2}=\lambda_{2}$. The number of the basis functions is chosen to be 110 for the TE case and $11+$ for 


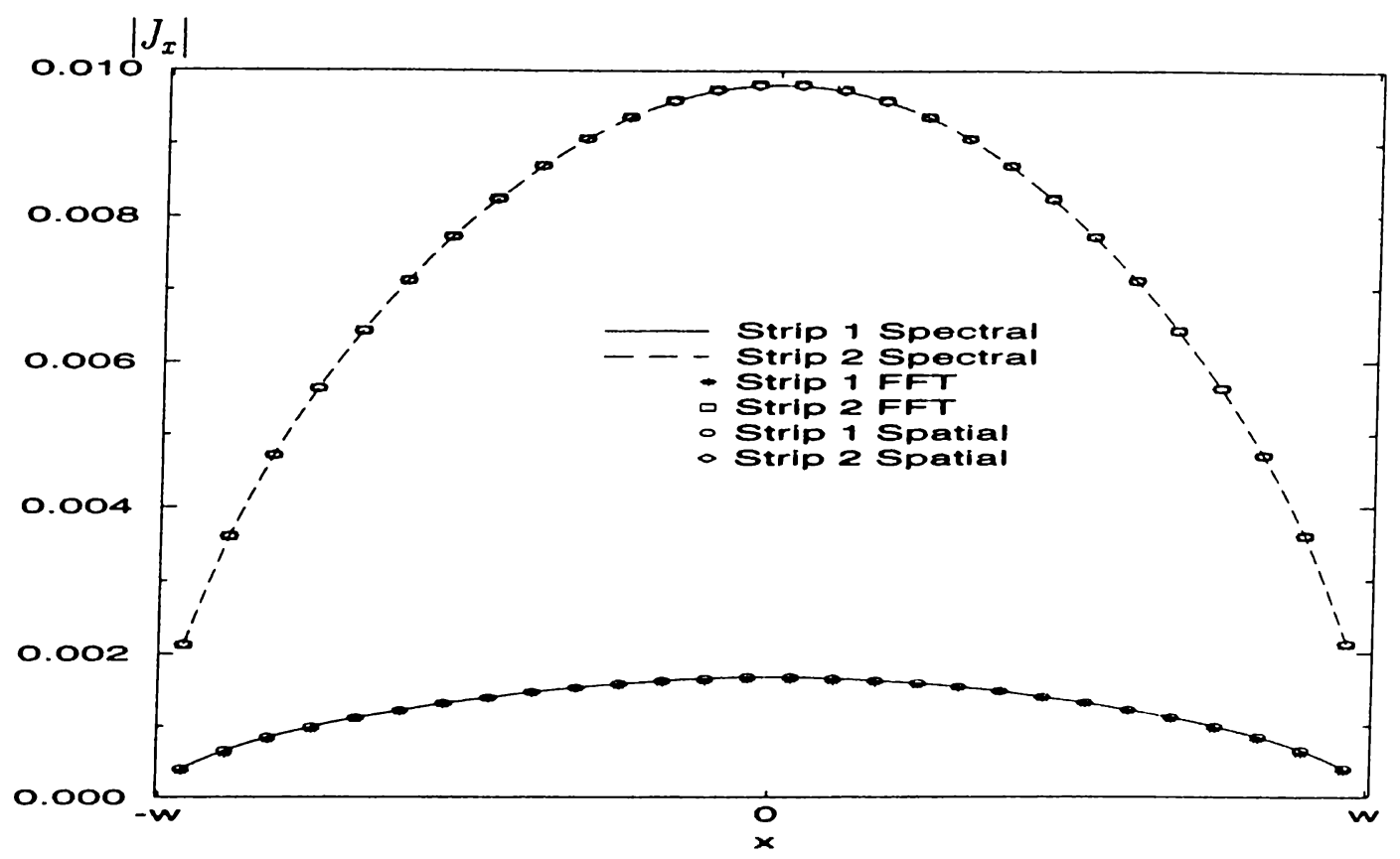

Figure 4.23: The magnitudes of the current densities on the two strips for the TM excitation

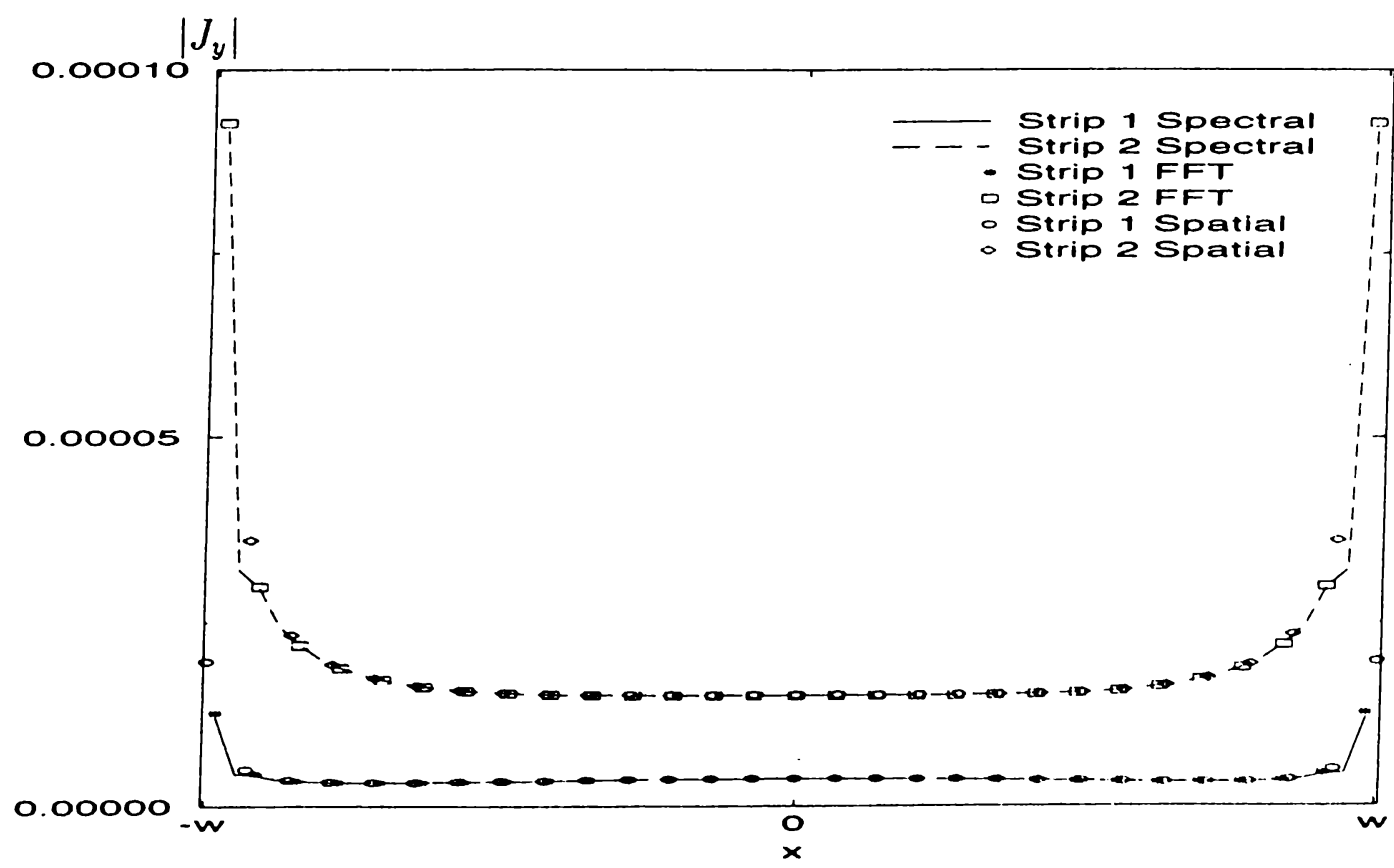

Figure 4.24: The magnitudes of the current densities on the two strips for the TM excitation 
the TM case, and the angle of incidence $\theta=0^{\circ}$. Table 4.3 shows the CPL times of

\begin{tabular}{||c|c|c|}
\hline \multirow{2}{*}{} & \multicolumn{2}{|c||}{ CPU time (s) } \\
\cline { 2 - 3 } & TE & TM \\
\hline Spectral & 109.2 & 63.6 \\
\hline FFT & 142.2 & 166.5 \\
\hline Spatial & 6980.2 & 2187.1 \\
\hline
\end{tabular}

Table 4.3: The CPU times of the spectral domain, the spatial domain and the FFT approaches for the TE and TM excitations

all methods for the TE and TM excitations, again the CPU time of the spectral domain approach is the smallest. Figs. 4.23 and 4.24 show the magnitudes of the current densities, obtained with using these three approaches, on the strips for the TE and TM excitations, respectively, no distinguishable difference in the results are observed.

\subsection{Examples for the MoM Formulation in the Spectral Domain}

After having established the superiority in the computational efficiency of the spectral-domain technique over the other two techniques for planar geometries in multilayer media. next two examples are investigated by the spectral-domain approach only:

The geometry of the first example is given in Fig. 4.25. where the angle of incidence $\theta=-45^{\circ}$. the medium is homogeneous with $\epsilon_{r}=1, k u=k d$. and there are 10 strips altogether. The magnitudes and phases of the current densities are given in Figs. 4.26-4.30 for the TE excitation. and in Figs. 4.31-1.35 for the TM excitation for five different strip widths, $k w=1.2 .3 .4 .5$. The 


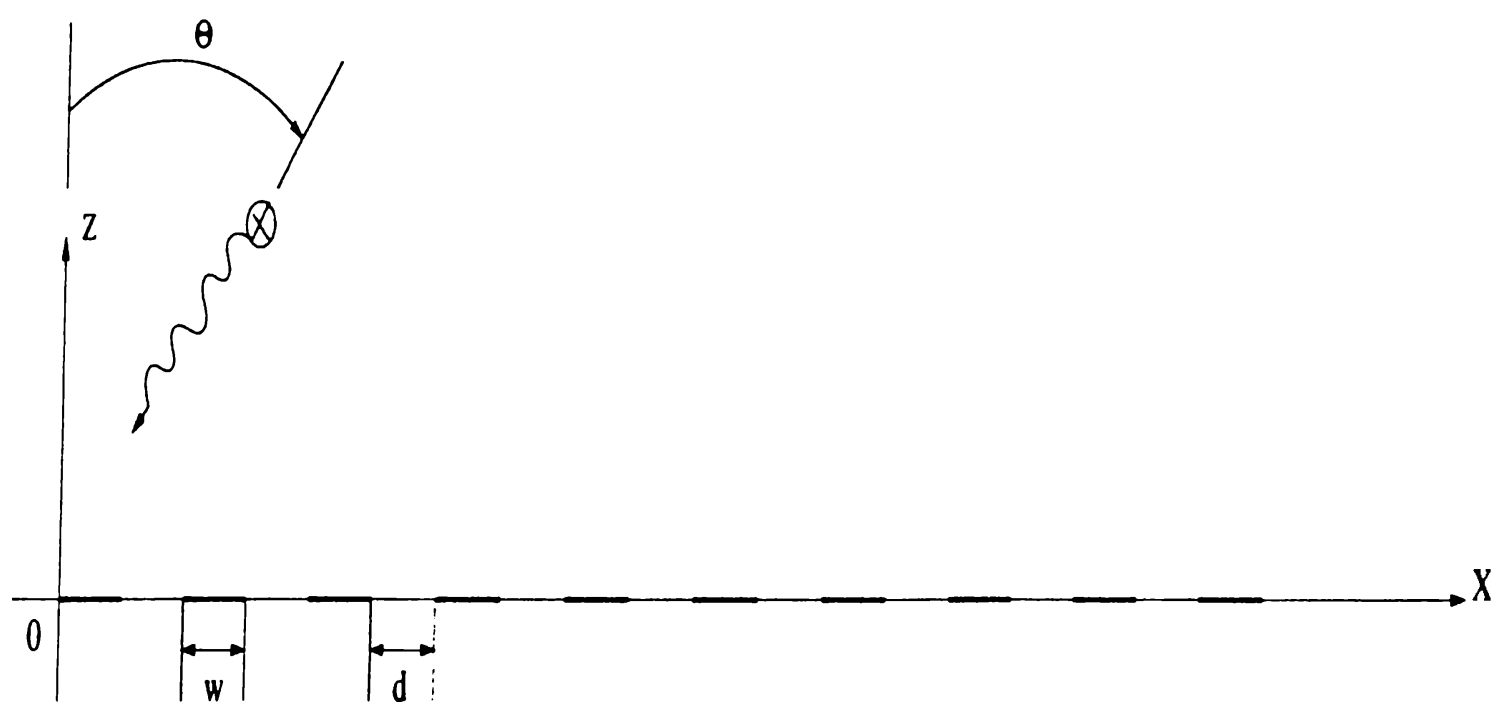

Figure 4.25: A geometry of ten strips located side by side in a homogeneous medium.

results are compared to those found in the literature [19], and agreement is quite good. Note that. in Figs. 4.26-4.35, solid lines and the triangular marks show the magnitude and the phase of the current densities, respectively. The magnitudes of the current densities in Figs. 4.26-4.35 are normalized so that thay can be put on the same plot with the phases of the current densities as in the literature [19]. 


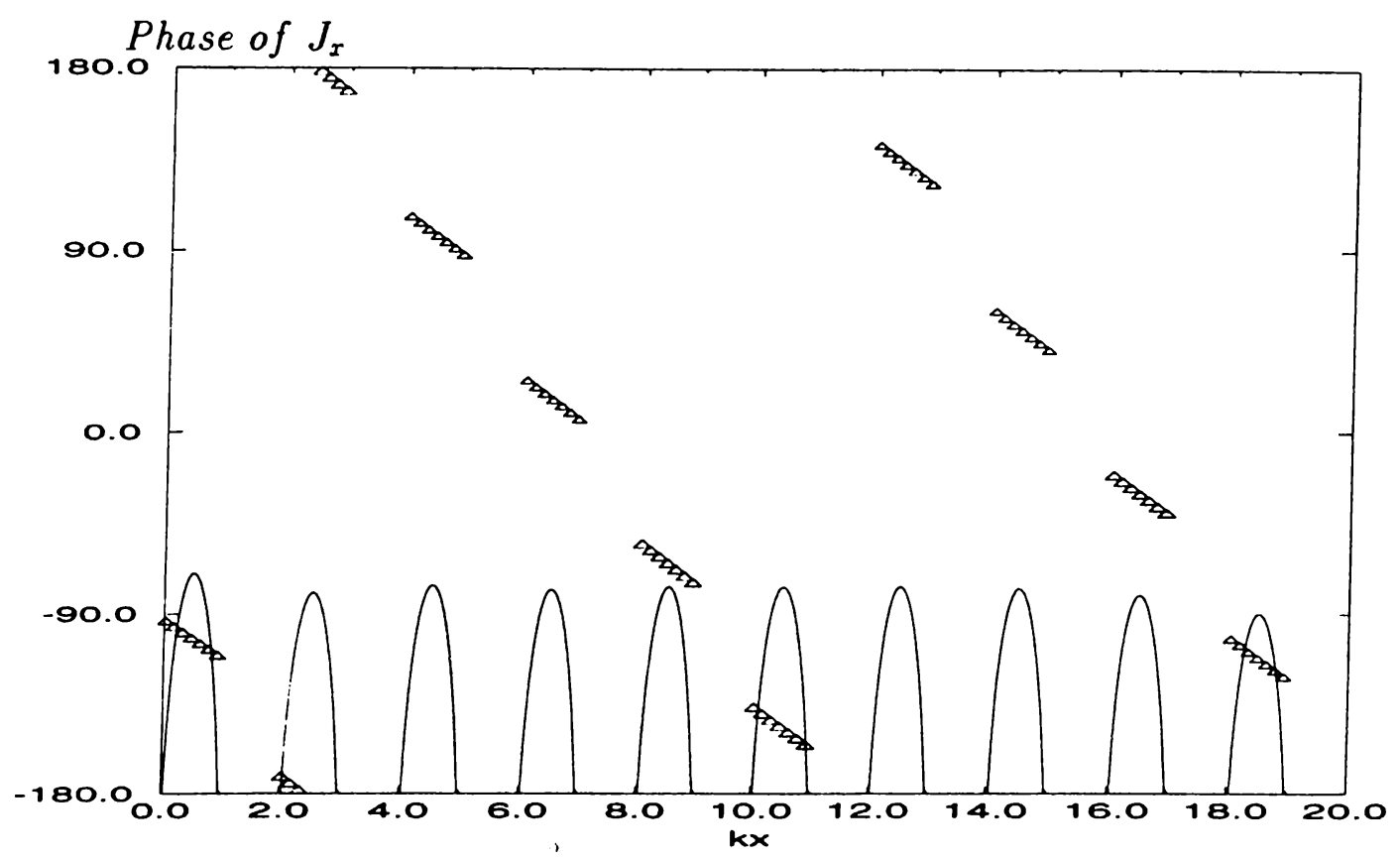

Figure 4.26: The magnitude and phase of the current densities for the TE excitation, and for $k w=1.0$

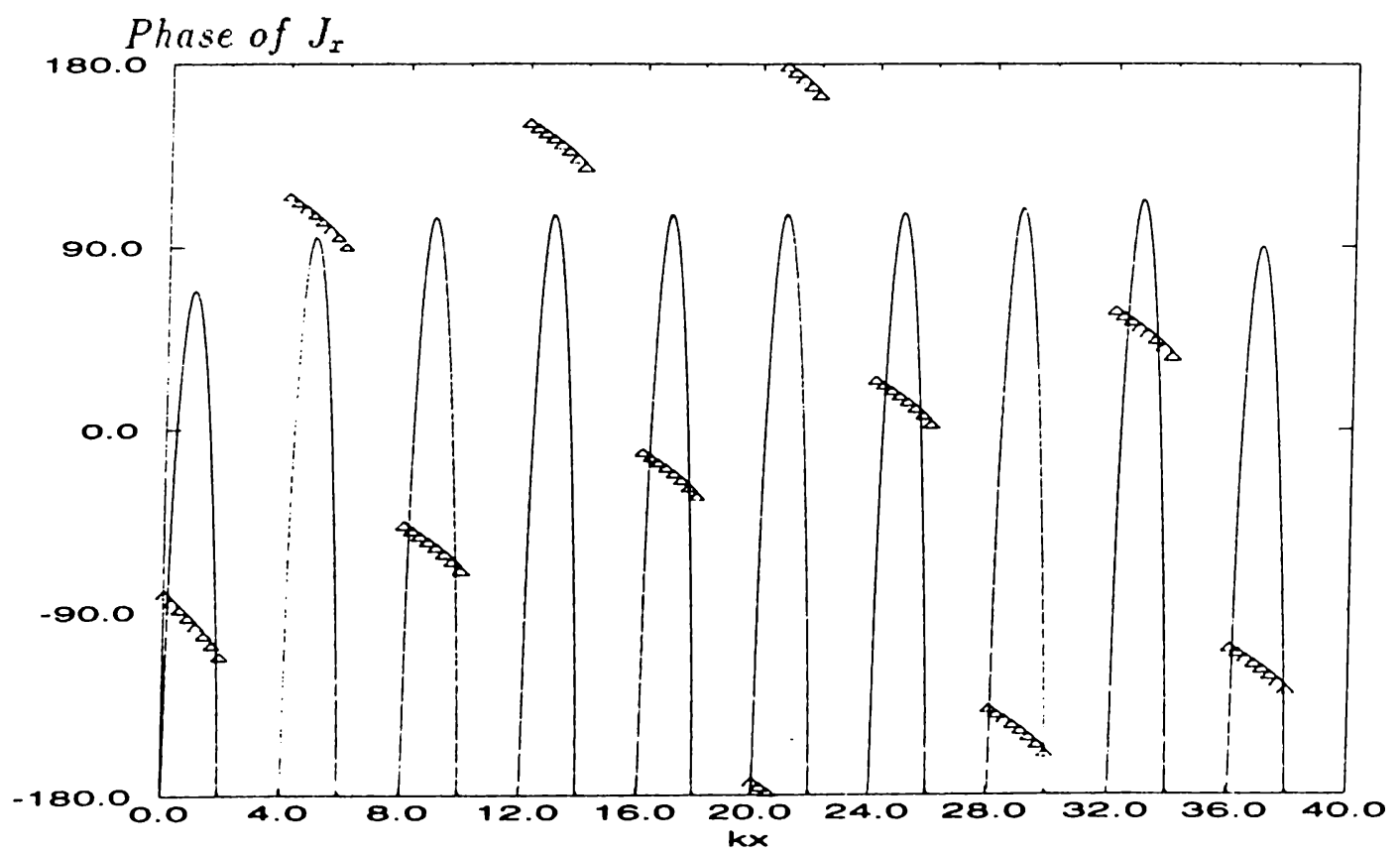

Figure 4.27: The magnitude and phase of the current densities for the TE excitation, and for $k u=2.0$ 


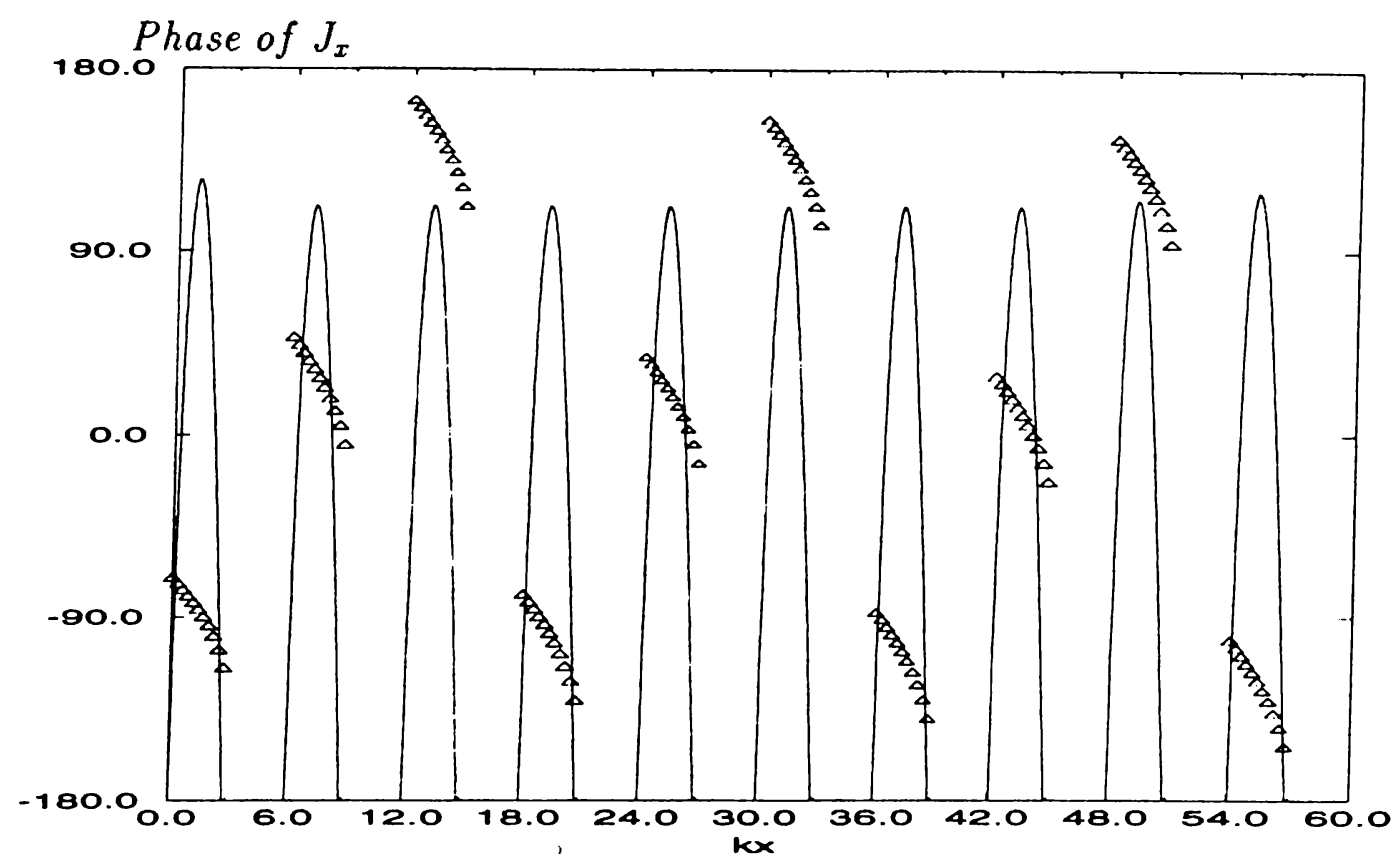

Figure 4.28: The magnitude and phase of the current densities for the TE excitation, and for $k w=3.0$

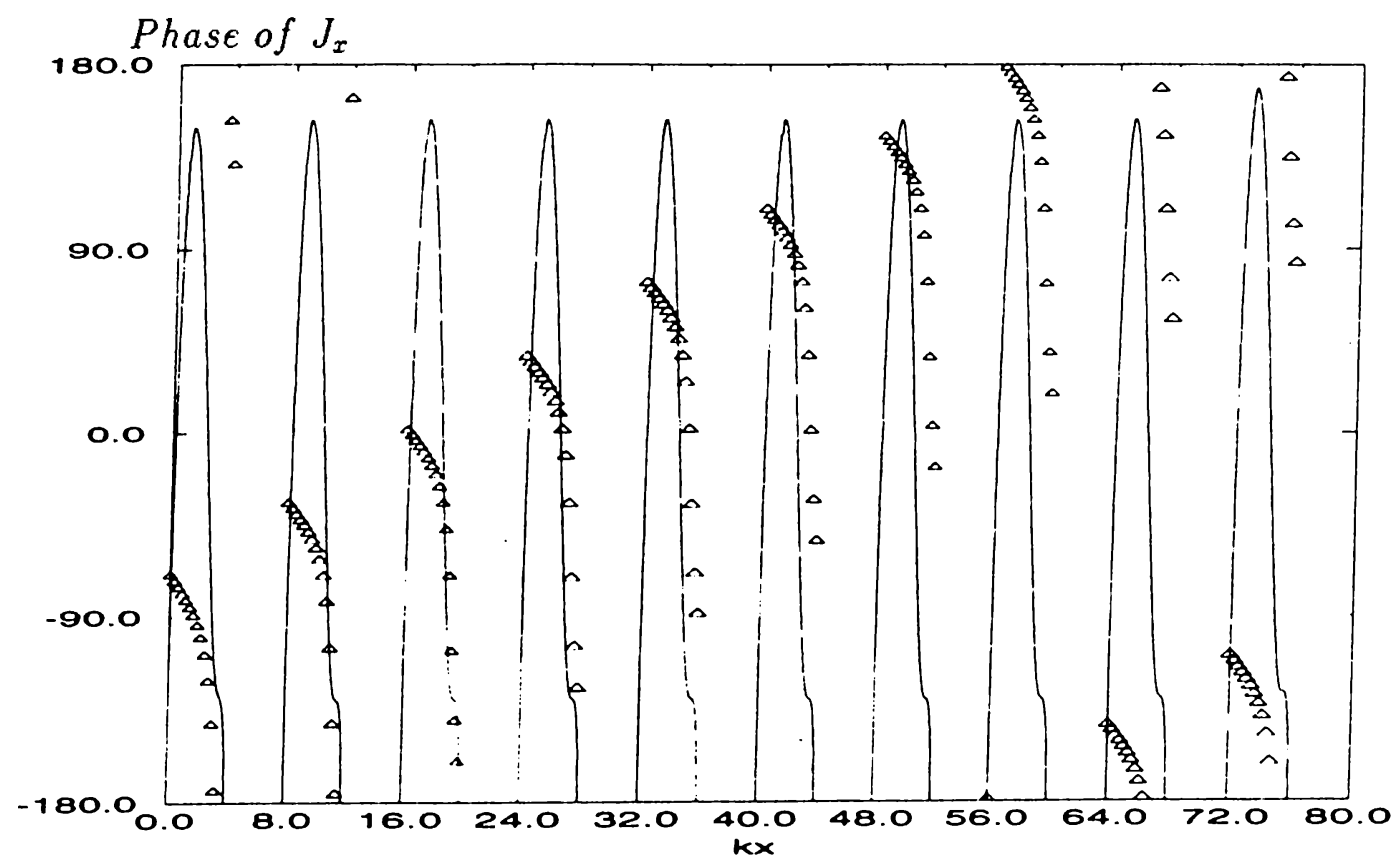

Figure 4.29: The magnitude and phase of the current densities for the TE excitation. and for $k w=4.0$ 


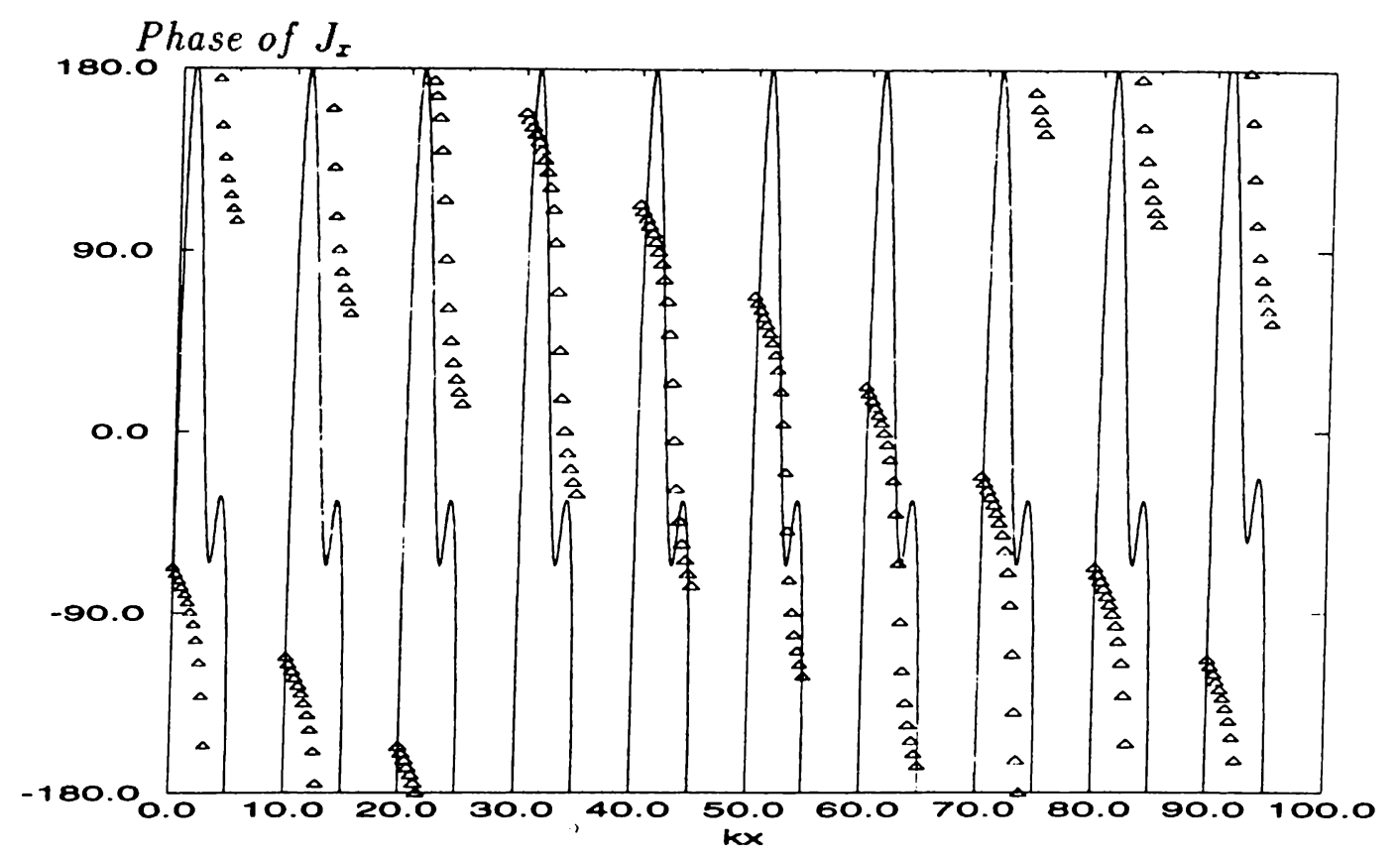

Figure 4.30: The magnitude and phase of the current densities for the TE excitation, and for $k w=5.0$

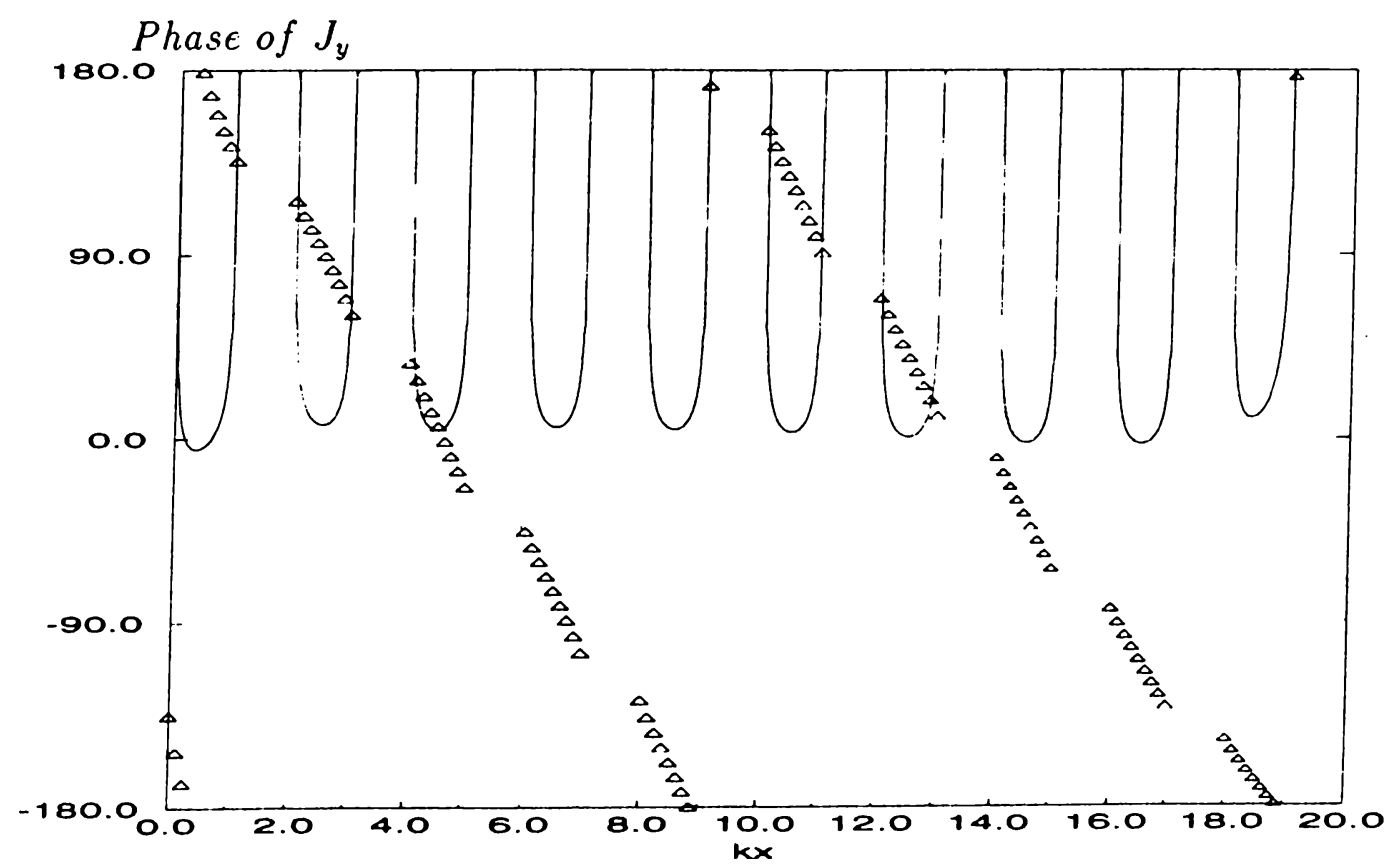

Figure 4.31: The magnitude and phase of the current densities for the T.M excitation. and for $k w=1.0$ 


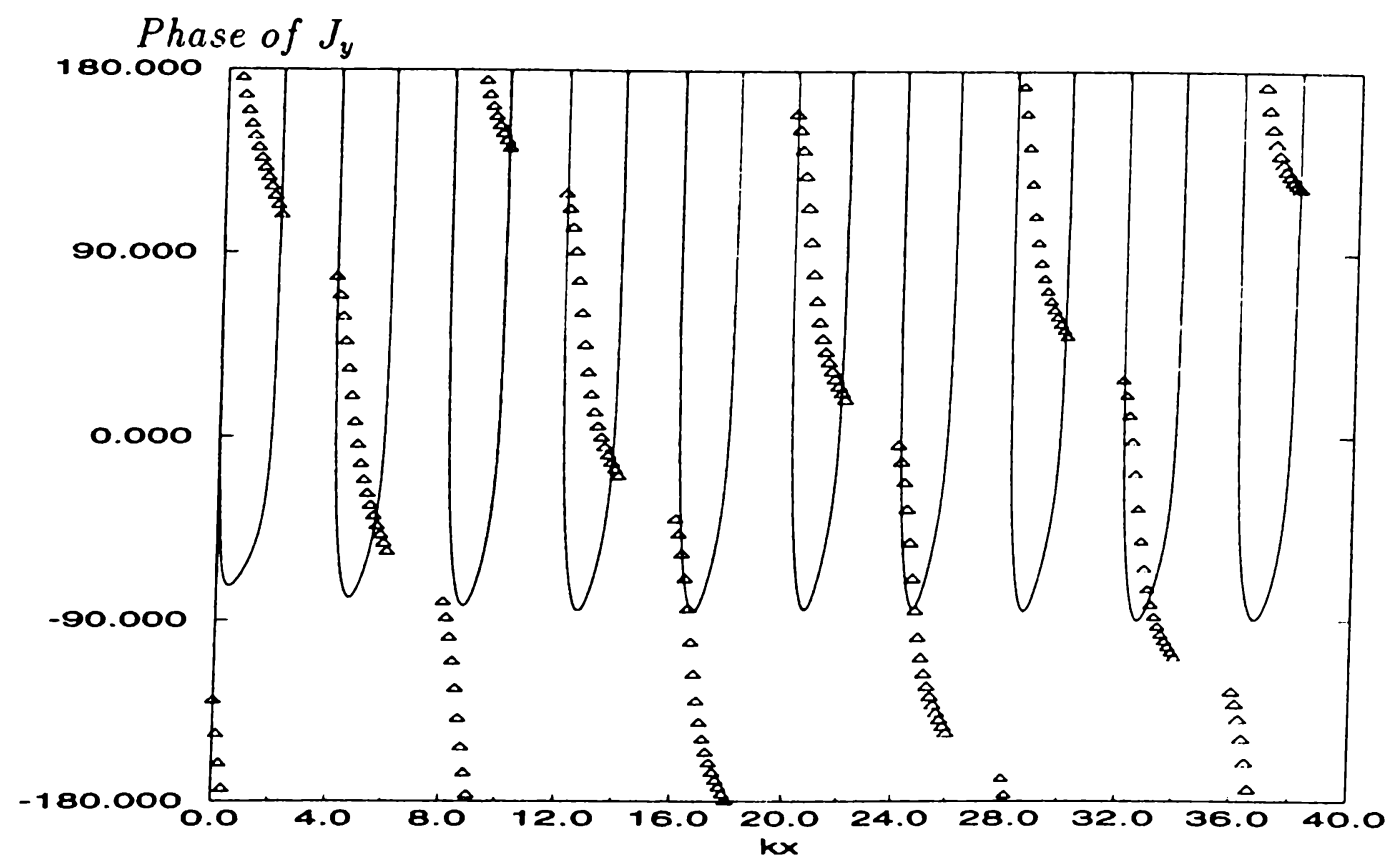

Figure 4.32: The magnitude and phase of the current densities for the TM excitation, and for $k w=2.0$

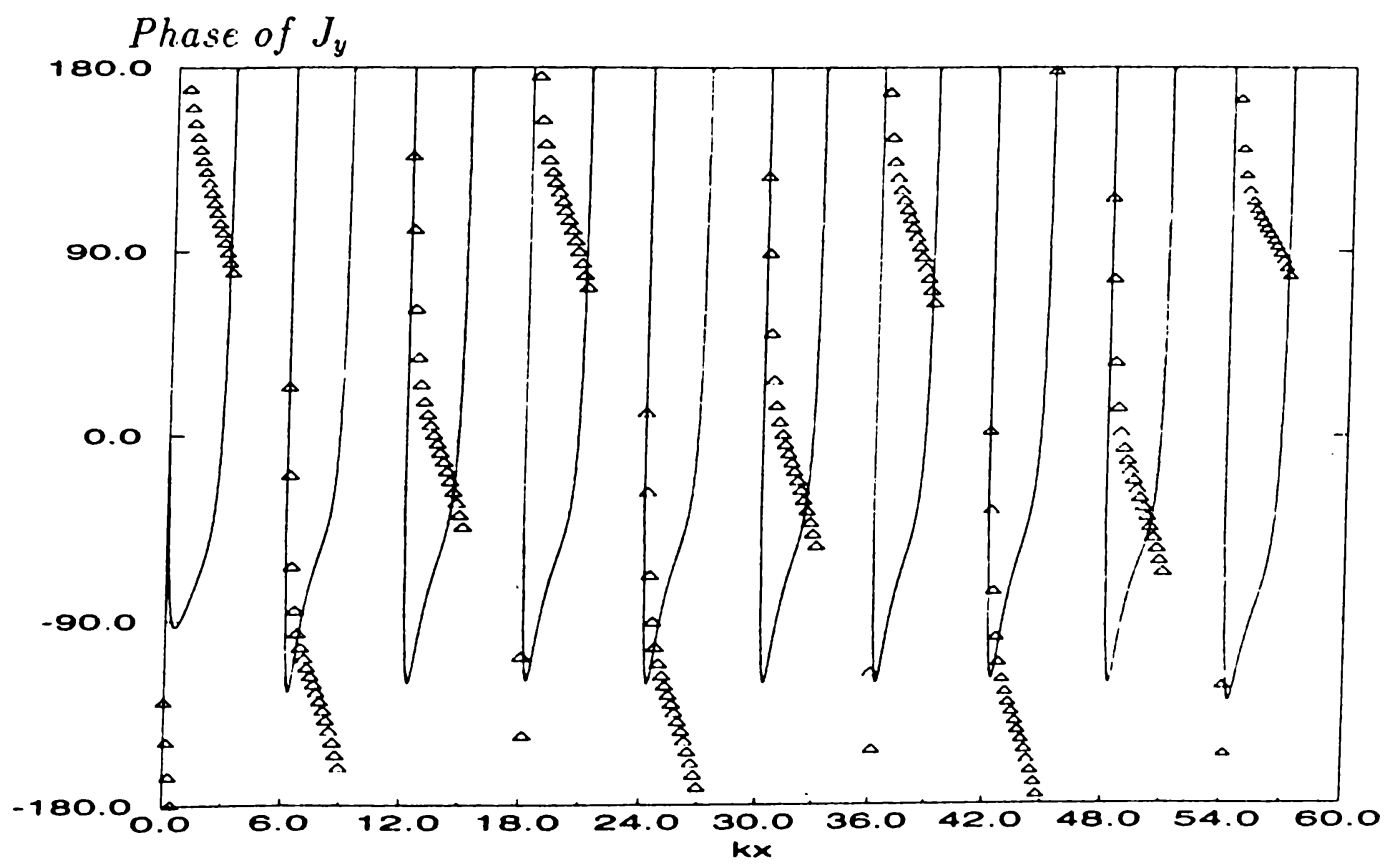

Figure 4.33: The magnitude and phase of the current densities for the $7 \mathrm{M}$ excitation, and for $k u=3.0$ 


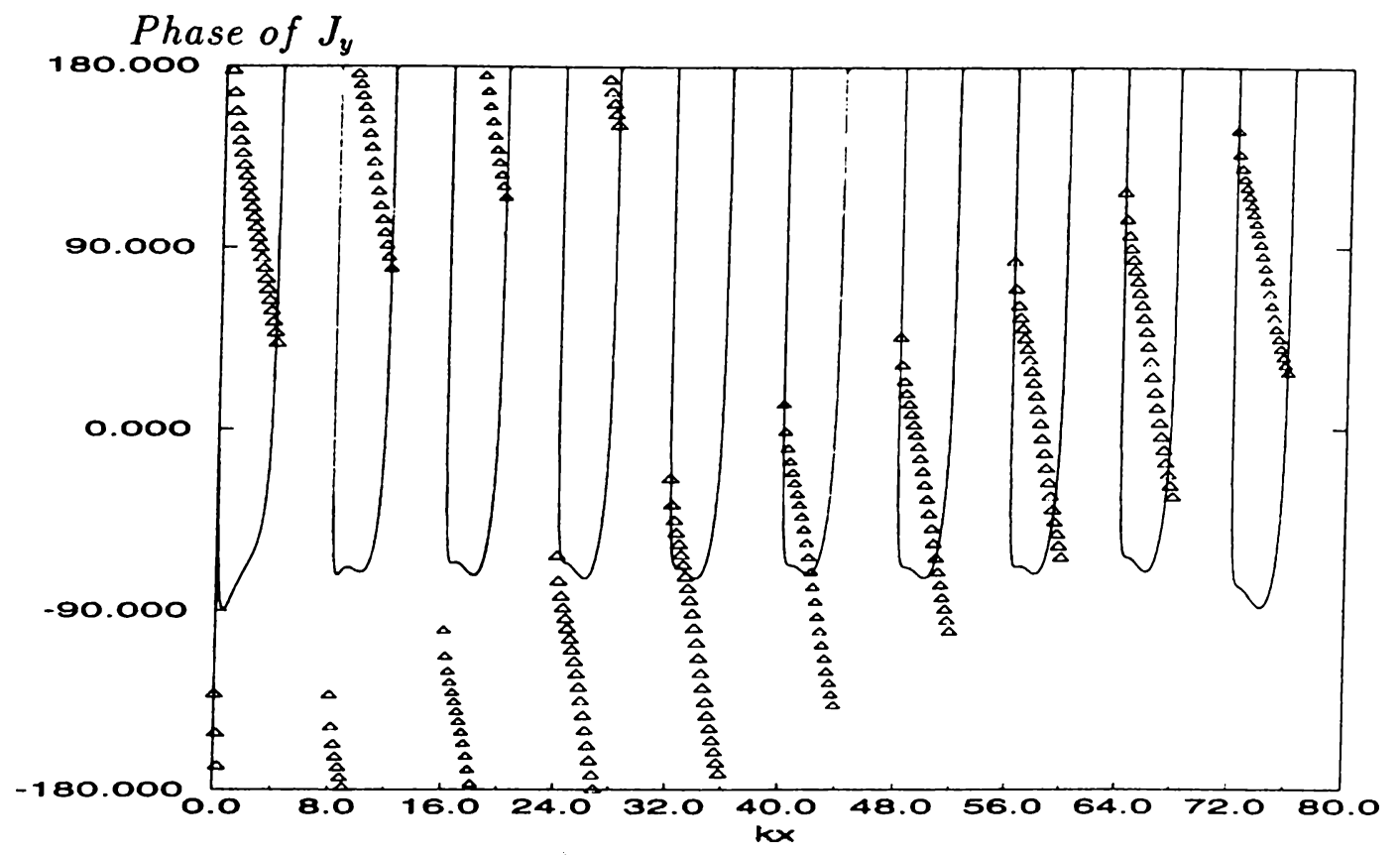

Figure 4.34: The magnitude and phase of the current densities for the TM excitation, and for $k w=4.0$

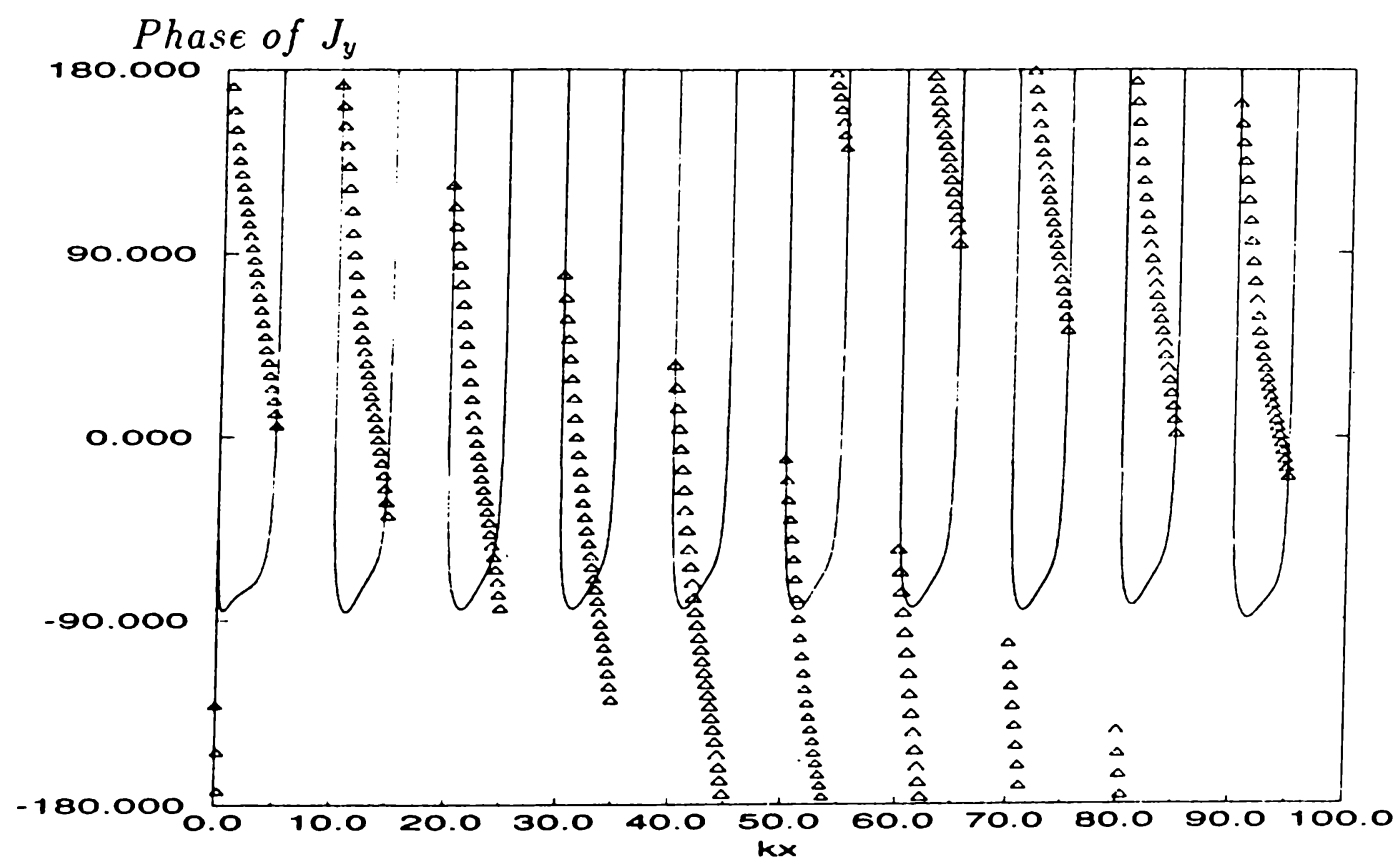

Figure 4.35: The magnitude and phase of the current densities for the TM excitation, and for $k w=5.0$ 


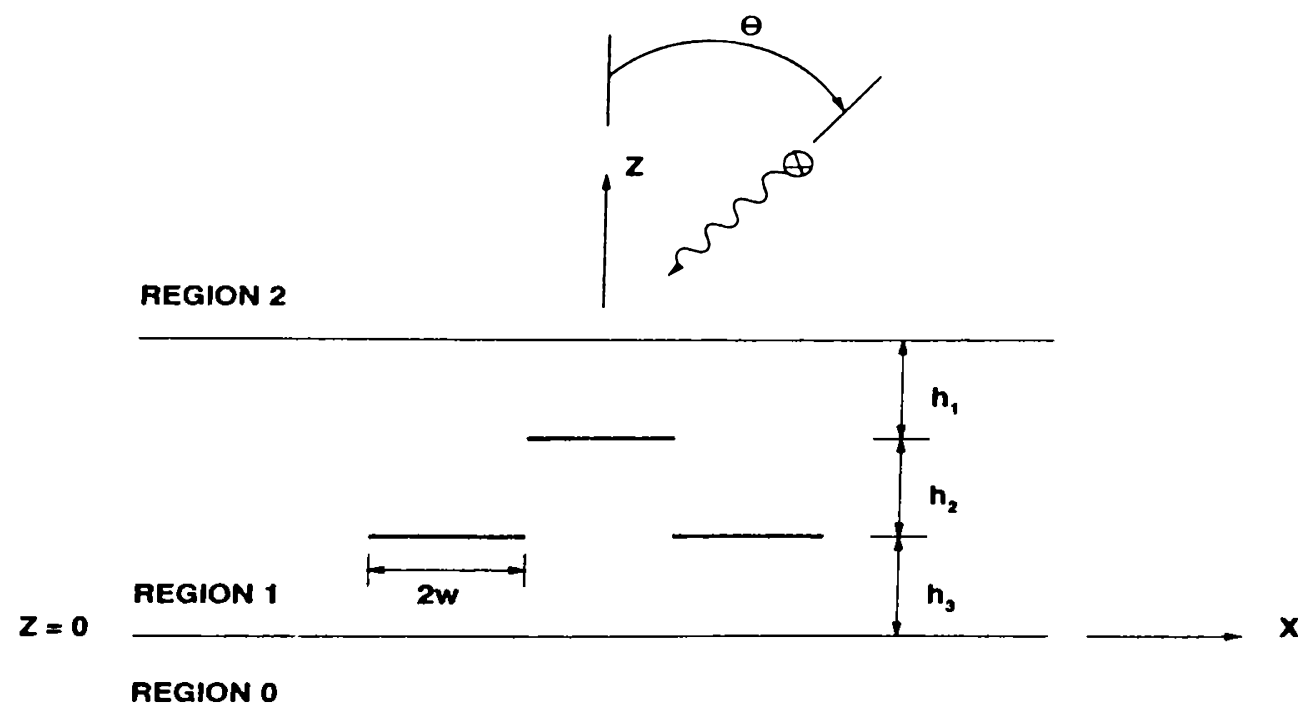

Figure 4.36: Three strips located in the same medium

The last example is a three-strip geometry in a three layer medium as shown in Fig. 4.36, for which Region 0 and Region 2 are free space. $\epsilon_{r_{1}}=4$ and $\epsilon_{r_{0}}=$ $\epsilon_{r_{2}}=1$, the width of the strips. $2 w=0.2 \lambda_{2}$, and $h_{1}=h_{2}=h_{3}=0.1 \lambda_{2}$. The number of the basis functions is chosen to be 105 for the TE case and 111 for the TM case, and the angle of incidence. $\theta=0^{\circ}$.

The CPU time to fill the impedance matrix and the source vector is $106.4 \mathrm{sec}$. for the TE case and $71.9 \mathrm{sec}$. for the TM case. The magnitudes of the current densities on the strips are given in Figs. 4.37 and 4.38 on the strips for the TE and TM excitations, respectively: 


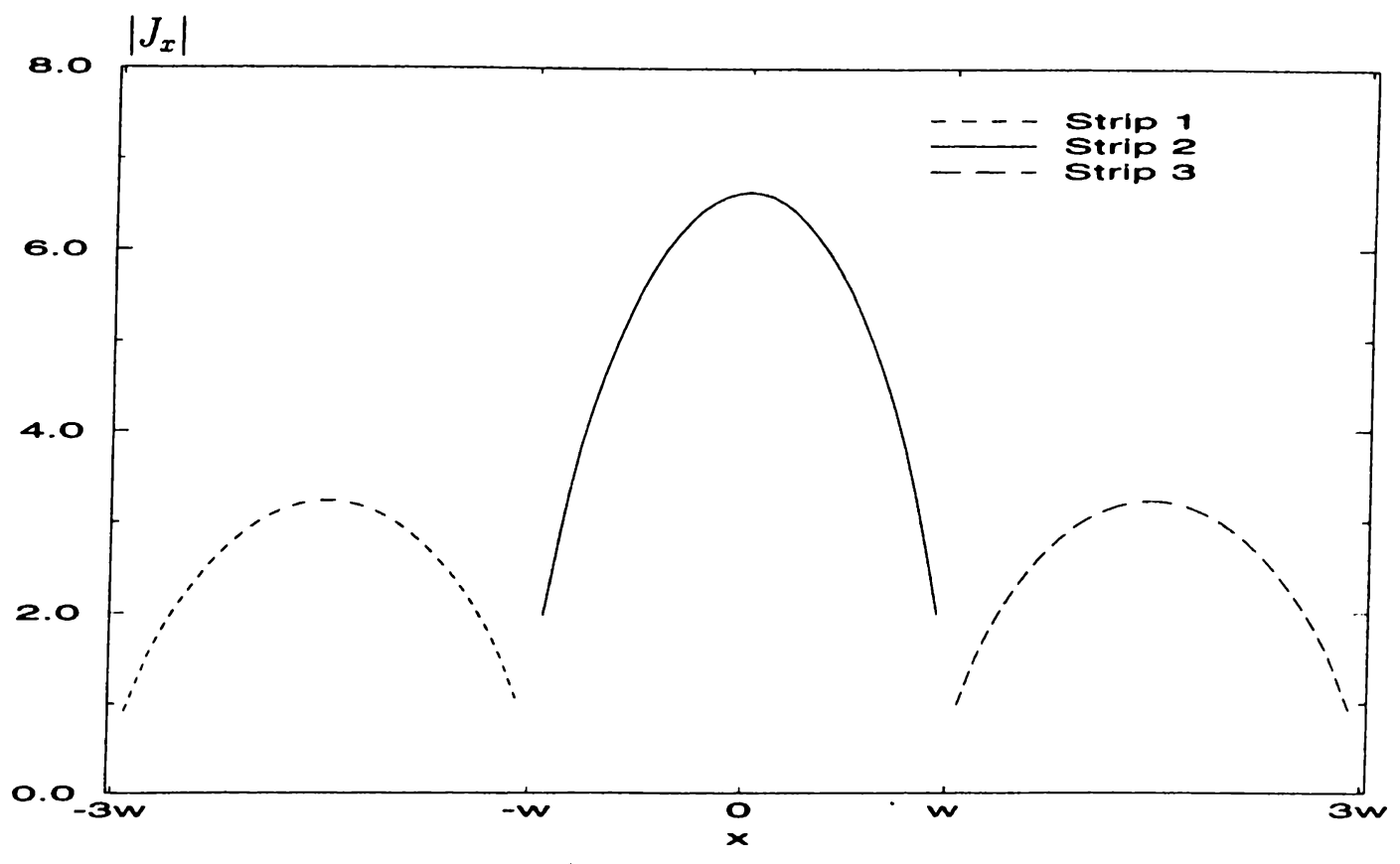

Figure 4.37: The magnitudes of the current densities on the three strips for the TE excitation

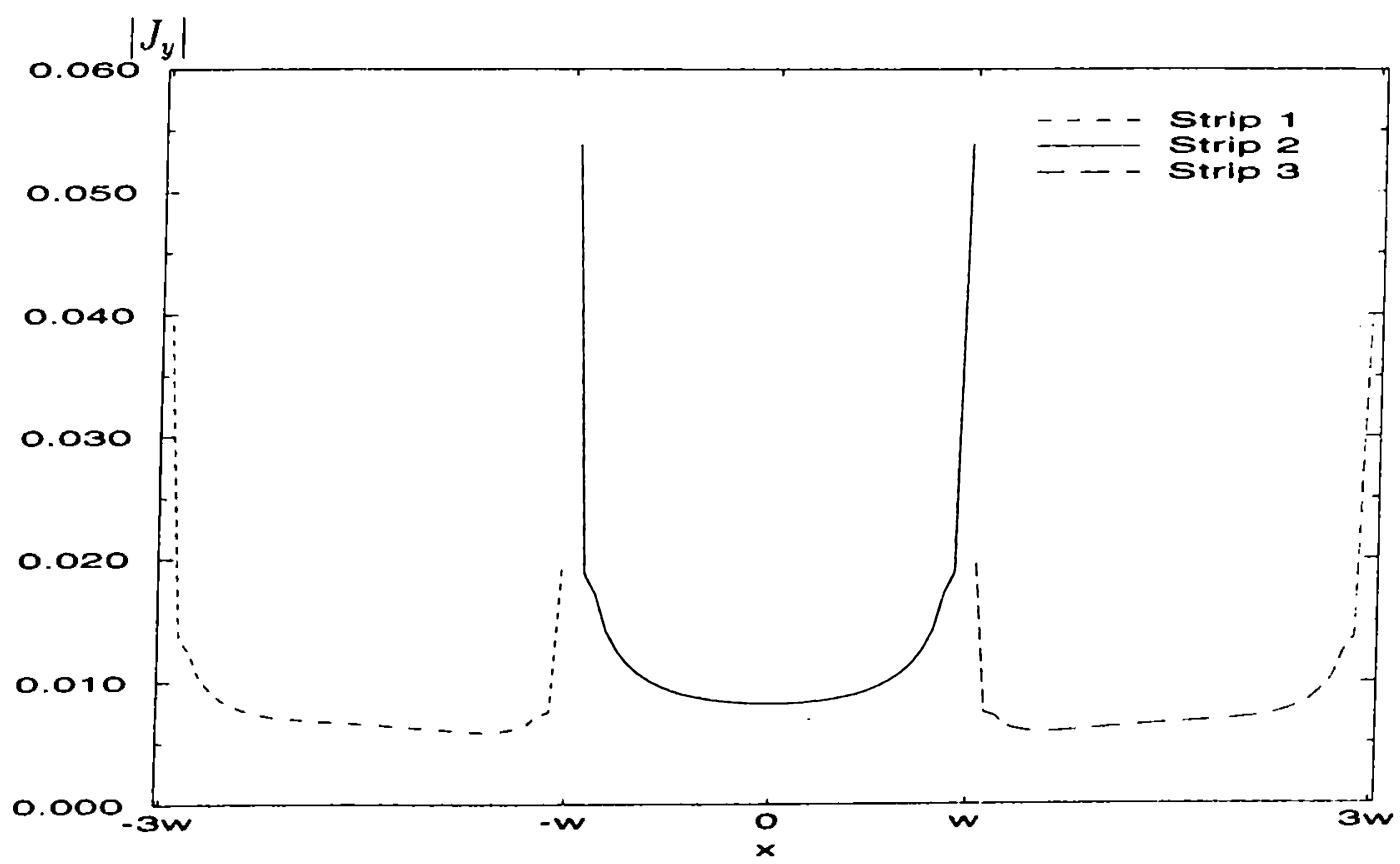

Figure 4.38: The magnitudes of the current densities on the three strips for the TM excitation 


\section{Chapter 5}

\section{Solving Linear Equations by the Iterative Method}

The basic idea of the Method of Moments is to transform an integral equation into a matrix equation and the numerical efficiency of the method is very sensitive to the technique used for the solution of the matrix equation. In this chapter, solutions for the linear system of equations of the type $A x=b$ are discussed where $A . b$ and $x$ correspond to impedance matrix. source and current density vectors in the Mo.M formulation, respectively:

Numerical methods used for solving linear system of equations can be classified into two types. namely direct and iterative methods. Direct methods are those yielding the exact solution in a finite number of elementary operations. The most commonly used direct method is the Gauss elimination. but even for this technique various choices of improved versions have been developed and they vary in computational efficiency and accuracy [20].

Iterative methods are those which start with an initial approximation for the 
solution and iteratively lead to better approximations. Iterative methods vary with the algorithm chosen and their rates of convergence.

Matrices associated with the linear systems are also classified as dense and sparse matrices. Dense matrices have very few zero elements. The matrix equations considered in this thesis form dense matrices.

In matrix equations, when the number of unknowns increases, matrix solution time dominates to the total CPU time which is the summation of the solution and matrix-fill times. In this thesis, a new iterative technique is proposed to reduce the matrix solution time which is an important consideration in the solution of electrically large geometries. The technique is based on dividing a large object into subregions and solving the current distribution on each subregion by considering the effects of other regions. The process should be repeated iteratively until a convergence is reached.

The example geometries are chosen to be planar surfaces in order to employ MoM formulation in the spectral domain. To assess the efficiency of the method. it is compared with LU decomposition. In the algorithm of the iterative method. LU decomposition is also used to find the result of subregions in each iteration. To demonstrate the algorithm. a single strip geometry is considered in $2-\mathrm{D}$. The strip is divided into $V_{2}$ subregions with $V_{1}$ unknowns in each subregion resulting total $N=N_{1} \times N_{2}$ number of unknowns. The equations. obtained from each row of the matrix. to be solved are of the form:

$$
\sum_{m=M_{a}}^{M I_{b}}-j w I_{y m}\left\langle\dot{T}_{y n} \cdot \dot{G}_{y y}^{A} \dot{B}_{m y}\right\rangle=\sum_{\substack{l=1 \\ l \neq m}}^{N} j \omega I_{y m}\left\langle\dot{T}_{y n} \cdot \dot{C}_{y y}^{A} \dot{B}_{l y}\right\rangle+b_{y n}
$$

Where $n$th component of the source vector is

$$
b_{y n}=-E_{1} 2 \pi \epsilon^{j k \sin \theta_{5 n_{2}}}\left(c^{j k \cos \theta=}+\dot{R}_{T E}^{i, i-1} \epsilon^{-j k \cos \theta z}\right) h_{2} \cdot \sin c\left(\frac{k \cdot \sin \theta h_{x}}{2}\right)
$$


for TM excitation and

$$
\sum_{m=1 M_{a}}^{M_{b}}-j \omega I_{x m}\left\langle\dot{T}_{x n}, \dot{G}_{x x}^{E} \tilde{B}_{m x}\right\rangle=\sum_{\substack{l=1 \\ l \neq m}}^{N} j \omega I_{x m}\left\langle\dot{T}_{x n} . \dot{G}_{x x}^{E} \dot{B}_{l x}\right\rangle+b_{x n}
$$

where $n$th component of the source vector is

$$
b_{x n}=\frac{k \cos \theta H_{i}}{\omega \epsilon} 2 \pi \epsilon^{j k \sin \theta x_{n_{2}}}\left(e^{j k \cos \theta z}-\tilde{R}_{T M}^{i, i-1} e^{j k \cos \theta z}\right) h_{x} \operatorname{sinc} c^{2}\left(\frac{k \sin \theta h_{x}}{2}\right)
$$

for TE excitation where $n=M_{a}, \cdots, M_{b}$ and $M_{b}-M_{a}+1=N_{1}$. The basis and testing functions are triangular functions for TE excitation and pulse functions for TM excitation. As it can be seen from Eqs. (5.1) and (5.3), the current distribution on each subregion can be obtained by solving this linear system. Then, to obtain the total current distribution, one should apply Eqs. (5.1) and (5.3), iteratively by changing $M_{a}$ and $M_{b}$. Changing $M_{a}$ and $M_{b}$ corresponds to changing the subregion. In this method, one iteration means $m$ is increased from one to $N$ and then it is decreased from $N$ to one.

In the solution of the linear system corresponding to each subregion, LU decomposition is employed. Since LU decomposition is $O\left(\mathrm{~N}_{1}^{3}\right)$ for each subregion the overall technique is $O\left(\Lambda_{1}^{3} \times N_{2} \times\right.$ number of ittrations $)$. On the other hand if the original matrix without any subdivision is solved using LL: decomposition. the technique is $O\left(\mathrm{~V}^{3}\right)$. Therefore, the proposed iterative technique improves the numerical efficiency because LU decomposition is applied to smaller size matrices.

The initial value of the unknown current coefficients is one of the important considerations of the method. The initial value can be set to unity: zero. a complex number or any constant then the iterative method will find the unknown coefficients. However. if a coarse solution is found before starting the iteration and this coarse solution is assigned as the initial value of the amplitudes of the basis functions. the convergence of the method will be improved. 
The comparison of $\mathrm{CPU}$ times between the iterative method and the $\mathrm{LU}$ decomposition method is presented in Section 5.1.

\subsection{Comparison of the Iterative Method and LU Decomposition}

In this section, three example geometries for both TM and TE excitation cases are studied using LU decomposition and iterative method. The CPU time comparisons are done increasing the number of unknowns, changing $N_{1}$ and $N_{2}$ without changing $N$. In the examples, the coarse solution is assigned as the initial value of the current and the size of the matrix for the coarse solution is chosen as $\mathrm{N}_{2}$ so when $N$ is changed, the coarse solution is changed.

\subsubsection{Example 1}

The geometry of first example is shown in Fig. 5.1. The parameters used in this example are as follows: $\epsilon_{r_{0}}=\epsilon_{r_{1}}=1$; the width of the strip, $2 u=1 \lambda_{1} ; h=0$ : the angle of incidence. $\theta=45^{\circ}$. Table 5.1 shows $N_{1}$ and $\Lambda_{2}$ values which are used in the example for various $N$ values. These $N_{1}$ and $N_{2}$ values are used to find the coarse solution for both TM and TE excitations.

Table 5.2 (a) shows the CPU times of the iterative method and LU decomposition for the TM excitation. It is observed that before $I=300$, the CPU time of $L U$ decomposition is less than the time of the iterative method. However, after $N=300$, the time of $L U$ decomposition increases very rapidly as compared to the time of the iterative method as shown in Table 5.2 (a). and required number of iterations in the iterative method increases slowly when the number of unknowns 

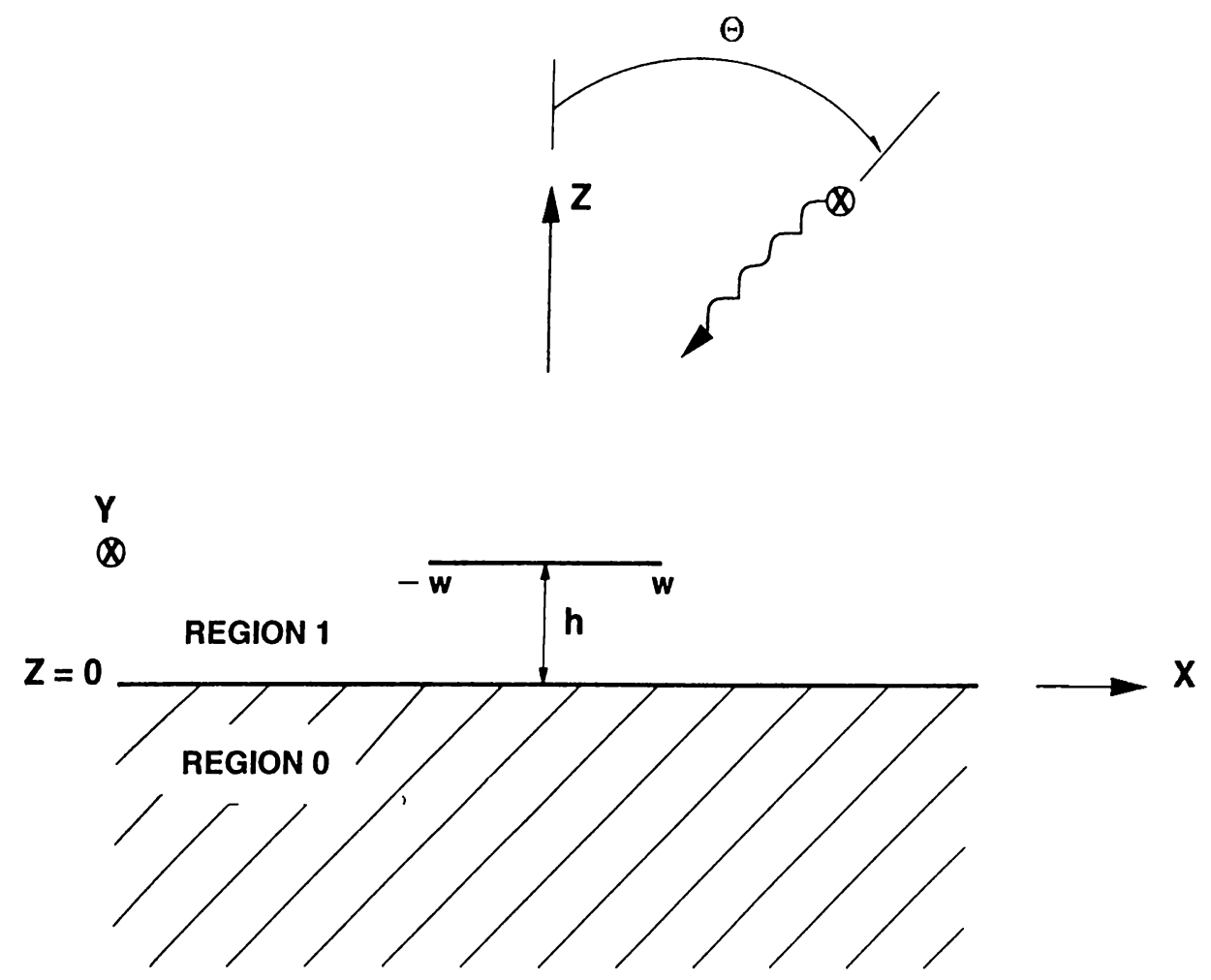

Figure 5.1: Strip of width $2 w$ located near interface between two semi-infinite half-spaces and illuminated by source in upper half-space

is increased, Table $5.2(\mathrm{~b})$.

It is known that $N=N_{1} \times N_{2}$ and in this study different $N_{1}$ and $N_{2}$ values are considered for the same $N$ to observe how the CPU time of the iterative method is sensitive to various $N_{1}$ and $\Lambda_{2}$ values. The results of a test case with $N$ is equal to 200 and 400 are shown in Table 5.3. It is observed that if $N_{1}$ is close to $N_{2}$, the CPU time is smaller, especially when $N_{2}$ is slightly greater than $N_{1}$ for the TM excitation case.

Table 5.4 (a) shows the CPU times of the iterative method and LU decomposition for the TE excitation case. It is observed that after $N$ is equal to 450 . the 


\begin{tabular}{||c|c|c||}
\hline $\mathrm{N}$ & $N_{1}$ & $N_{2}$ \\
\hline 50 & 5 & 10 \\
\hline 100 & 10 & 10 \\
\hline 150 & 10 & 15 \\
\hline 200 & 10 & 20 \\
\hline 250 & 10 & 25 \\
\hline 300 & 10 & 30 \\
\hline 350 & 10 & 35 \\
\hline 400 & 10 & 40 \\
\hline 450 & 10 & 45 \\
\hline 500 & 10 & 50 \\
\hline 600 & 20 & 30 \\
\hline 700 & 20 & 35 \\
\hline 800 & 20 & 40 \\
\hline 900 & 20 & 45 \\
\hline 1000 & 20 & 50 \\
\hline
\end{tabular}

Table 5.1: The values of $N_{1}$ and $N_{2}$ for different $N$ for example 1 .

C.PU time of the iterative method is less than the CPU time of LU decomposition. The number of iterations for the TE excitation is greater than the number for the TM excitation because of the oscillatory behavior of the current for the TE excitation.

In Table 5.5 (a) and (b), The CPU times and required number of iterations are compared for different $N_{1}$ and $N_{2}$ values corresponding to $N=200$ and $N=100$. respectively for the TE excitation. It is observed that the smallest value of the the CPU time is obtained for $N_{2}=10$ when $N=200$ and for $N_{2}=200$ when $N=400$. For $N=400$ case, the coarse solution is very close to the exact solution therefore the smallest value of the CPU time is achieved with the smallest number of iterations.

Figure 5.2 shows the improvement of the iterative method with respect to the iteration number when $N$ is equal to 1000 for the 'TM excitation. It is observed 
that the difference between the current magnitude for two iterations is small. As a result, the process is concluded at 10 iterations. The magnitude of the current densities for the iterative method and $L U$ decomposition are in agreement for the TM excitation in Fig. 5.3.

Figure 5.4 shows the improvement of the iterative method with respect to the iteration number when $N$ is equal to 1000 for the TE excitation. For this case, the difference between iterations is not small so the number of iterations for the TE excitation is greater than the number of iterations for the TM excitation. Fig. 5.5 compares the results of the iterative method and LU decomposition and a good agreement is observed between the two methods.

In the iterative method, the error is defined as

$$
\text { error }=\frac{\left\|x_{\text {old }}-x_{n e w}\right\|}{\left\|x_{n e w}\right\|}
$$

where $x_{\text {old }}$ and $x_{\text {new }}$ correspond to consecutive iterative results. The error plots of the iterative method for 1000 unknowns and for the TMI and TE excitations are shown in Fig. 5.6 and 5.7, respectively. The error plot shows the improvement. between two adjacent iterations and the error plot of the TM excitation reaches the error criteria faster than the error plot of the TE excitation since the number of iterations for the TE excitation is greater than the number of itcrations for the 'TM excitation. 


\begin{tabular}{||c|c|c||}
\hline \multirow{2}{*}{$\mathrm{N}$} & \multicolumn{2}{|c|}{ CPU time $(\mathrm{s})$} \\
\cline { 2 - 3 } & Ite. & $\mathrm{LU}$ \\
\hline 50 & 6.1 & 0.1 \\
\hline 100 & 6.4 & 0.4 \\
\hline 150 & 7.1 & 1.4 \\
\hline 200 & 7.9 & 3.3 \\
\hline 250 & 9.6 & 6.6 \\
\hline 300 & 9.7 & 11.6 \\
\hline 350 & 11.4 & 18.1 \\
\hline 400 & 11.6 & 27.0 \\
\hline 450 & 12.7 & 37.9 \\
\hline 500 & 14.3 & 51.9 \\
\hline 600 & 20.0 & 89.0 \\
\hline 700 & 23.6 & 141.2 \\
\hline 800 & 27.3 & 210.1 \\
\hline 900 & 32.4 & 298.2 \\
\hline 1000 & 43.0 & 405.6 \\
\hline
\end{tabular}

(a)

\begin{tabular}{||c|c||}
\hline $\mathrm{N}$ & Number of Iterations \\
\hline 50 & 6 \\
\hline 100 & $\mathrm{~T}$ \\
\hline 150 & $\mathrm{~T}$ \\
\hline 200 & $\mathrm{~T}$ \\
\hline 250 & 8 \\
\hline 300 & 8 \\
\hline 350 & 8 \\
\hline 400 & 8 \\
\hline 450 & 8 \\
\hline 500 & 8 \\
\hline 600 & 9 \\
\hline 700 & 9 \\
\hline 800 & 9 \\
\hline 900 & 9 \\
\hline 1000 & 10 \\
\hline
\end{tabular}

(b)

Table 5.2: (a) The CPU times of the iterative method and Lli decomposition (b) The number of iterations of the iterative method for the 'TM excitation and example 1. 


\begin{tabular}{||c|c|c|c||}
\hline$N_{1}$ & $N_{2}$ & CPU time (s) & Number of Iterations \\
\hline 2 & 100 & 9.4 & 6 \\
\hline 4 & 50 & 8.1 & 7 \\
\hline 5 & 40 & 8.1 & 7 \\
\hline 8 & 25 & 7.7 & 7 \\
\hline 10 & 20 & 8.0 & 7 \\
\hline 20 & 10 & 7.8 & 8 \\
\hline 25 & 8 & 8.0 & 9 \\
\hline 40 & 5 & 9.0 & 8 \\
\hline 50 & 4 & 9.2 & 8 \\
\hline 100 & 2 & 12.1 & 7 \\
\hline
\end{tabular}

(a)

\begin{tabular}{||c|c|c|c||}
\hline$N_{1}$ & $N_{2}$ & CPC time (s) & Number of Iterations \\
\hline 2 & 200 & 16.1 & 7 \\
\hline 4 & 100 & 12.2 & 7 \\
\hline 8 & 50 & 11.9 & 8 \\
\hline 10 & 40 & 11.6 & 8 \\
\hline 16 & 25 & 11.5 & 8 \\
\hline 20 & 20 & 12.3 & 9 \\
\hline 25 & 16 & 13.0 & 9 \\
\hline 40 & 10 & 15.5 & 9 \\
\hline 50 & 8 & 19.1 & 10 \\
\hline 100 & 4 & 30.8 & 9 \\
\hline 200 & 2 & 60.5 & 8 \\
\hline
\end{tabular}

(b)

Table 5.3: The CPU time and number of iterations of the iterative method for the $T M$ excitation when $\mathrm{N}$ is set to (a) 200. (b) 400 . 


\begin{tabular}{||c|c|c||}
\hline \multirow{2}{*}{$\mathrm{N}$} & \multicolumn{2}{|c|}{ CPU time (s) } \\
\cline { 2 - 3 } & Ite. & $\mathrm{LU}$ \\
\hline 50 & 6.7 & 0.1 \\
\hline 100 & 7.1 & 0.6 \\
\hline 150 & 14.7 & 1.4 \\
\hline 200 & 32.9 & 4.9 \\
\hline 250 & 38.3 & 10.1 \\
\hline 300 & 30.9 & 17.7 \\
\hline 350 & 57.5 & 28.7 \\
\hline 400 & 65.3 & 43.1 \\
\hline 450 & 56.6 & 68.9 \\
\hline 500 & 49.2 & 85.2 \\
\hline 600 & 52.1 & 149.8 \\
\hline 700 & 107.6 & 240.7 \\
\hline 800 & 492.7 & 363.6 \\
\hline 900 & 868.8 & 1597.3 \\
\hline 1000 & 203.6 & 2179.5 \\
\hline
\end{tabular}

(a)

\begin{tabular}{||c|c||}
\hline$X$ & Number of Iterations \\
\hline 50 & 16 \\
\hline 100 & 12 \\
\hline 150 & 97 \\
\hline 200 & 190 \\
\hline 250 & 144 \\
\hline 300 & 80 \\
\hline 350 & 126 \\
\hline 400 & 113 \\
\hline 450 & 77 \\
\hline 500 & 52 \\
\hline 600 & 35 \\
\hline 700 & 60 \\
\hline 800 & 184 \\
\hline 900 & 111 \\
\hline 1000 & 58 \\
\hline
\end{tabular}

(b)

Table 5.4: (a) The CPU times of the iterative method and LU decomposition (b) The number of iterations of the iterative method for the TE excitation and example 1. 


\begin{tabular}{||c|c|c|c||}
\hline$N_{1}$ & $N_{2}$ & CPU time $(\mathrm{s})$ & Number of Iterations \\
\hline 2 & 100 & 23.3 & 83 \\
\hline 4 & 50 & 113.6 & 721 \\
\hline 5 & 40 & 132.6 & 848 \\
\hline 8 & 25 & 58.1 & 325 \\
\hline 10 & 20 & 37.1 & 190 \\
\hline 20 & 10 & 8.8 & 11 \\
\hline 25 & 8 & 9.0 & 11 \\
\hline 40 & 5 & 22.1 & 9 \\
\hline 50 & 4 & 9.1 & 8 \\
\hline 100 & 2 & 12.3 & 7 \\
\hline
\end{tabular}

(a)

\begin{tabular}{||c|c|c|c||}
\hline$N_{1}$ & $N_{2}$ & CPU time (s) & Number of Iterations \\
\hline 2 & 200 & 13.8 & 2 \\
\hline 4 & 100 & 33.7 & 9 \\
\hline 8 & 50 & 43.3 & 65 \\
\hline 10 & 40 & 71.5 & 113 \\
\hline 16 & 25 & 53.0 & 78 \\
\hline 20 & 20 & 38.5 & 47 \\
\hline 25 & 16 & 28.3 & 31 \\
\hline 40 & 10 & 18.6 & 11 \\
\hline 50 & 8 & 20.6 & 12 \\
\hline 100 & 4 & 30.8 & 9 \\
\hline 200 & 2 & 59.4 & 8 \\
\hline
\end{tabular}

(b)

Table 5.5: The CPU time and number of iterations of the iterative method for the TE excitation when $V$ is set to (a) 200. (b) 400 . 


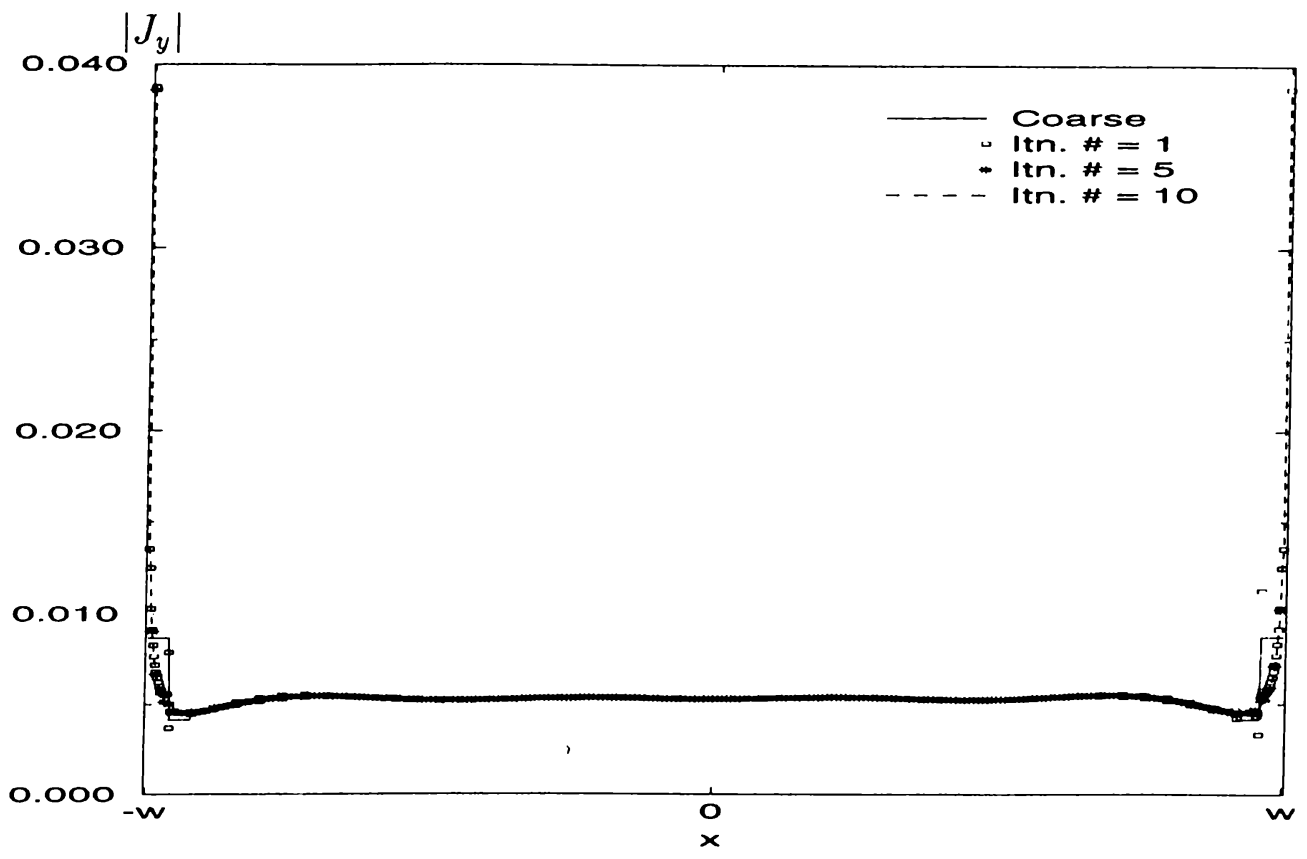

Figure 5.2: The current magnitude of the iterative method for the TM excitation when iteration number is increasing for $N=1000$

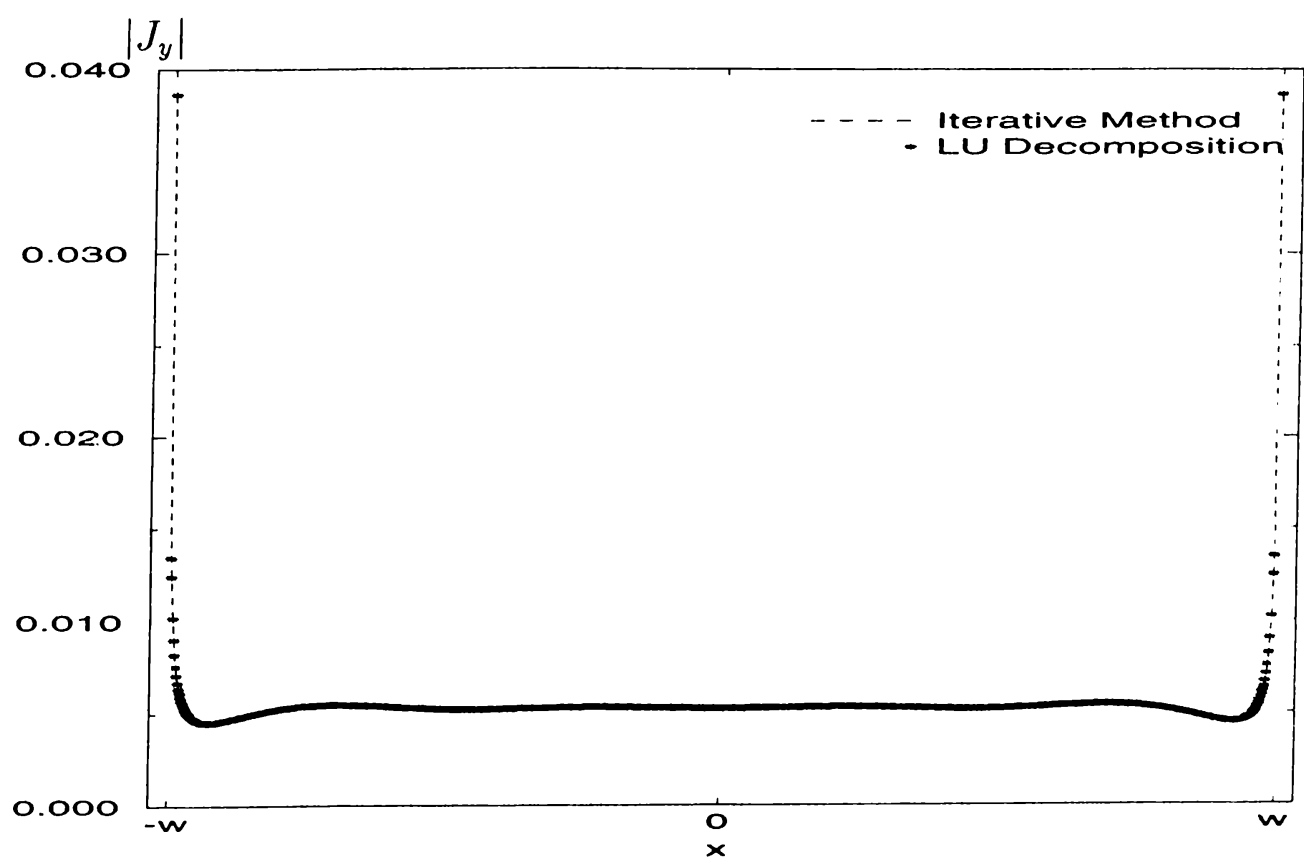

Figure 5.3: The current magnitude of the iterative method and LU decomposition for the TM excitation and for $N=1000$. 


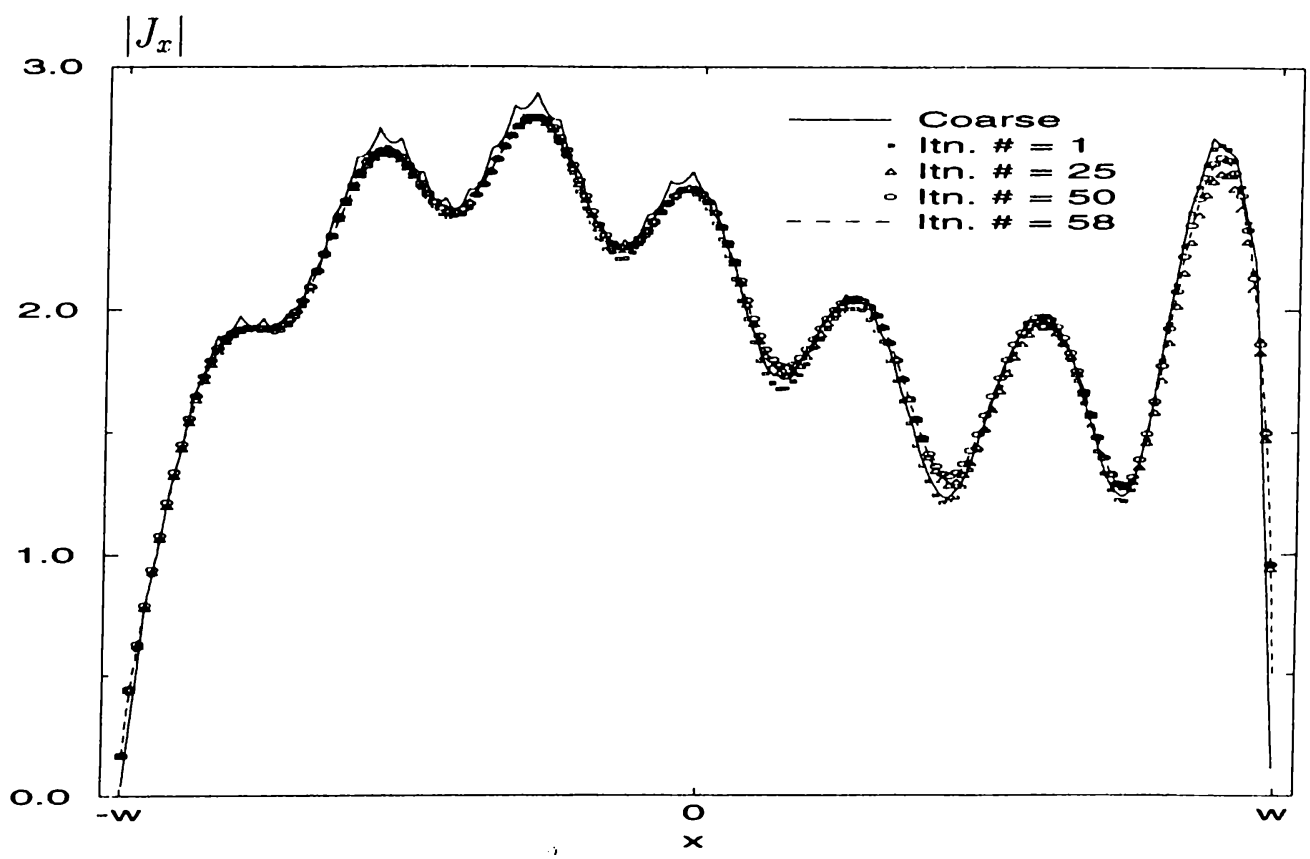

Figure 5.4: The current magnitude of the iterative method for the TE excitation when iteration number is increasing for $N=1000$

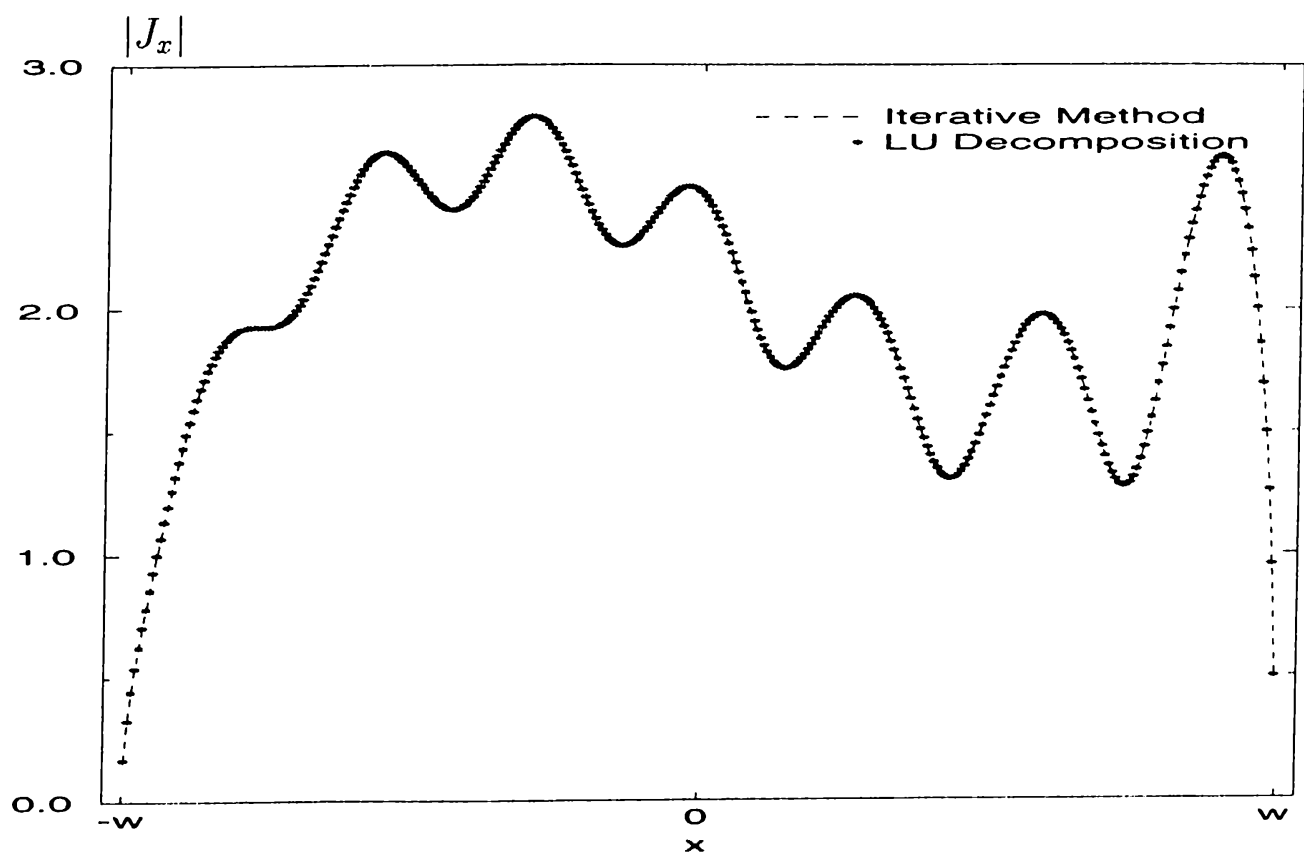

Figure 5.5: The current magnitude of the iterative method and LU' decomposition for the TE excitation and for $N=1000$ 


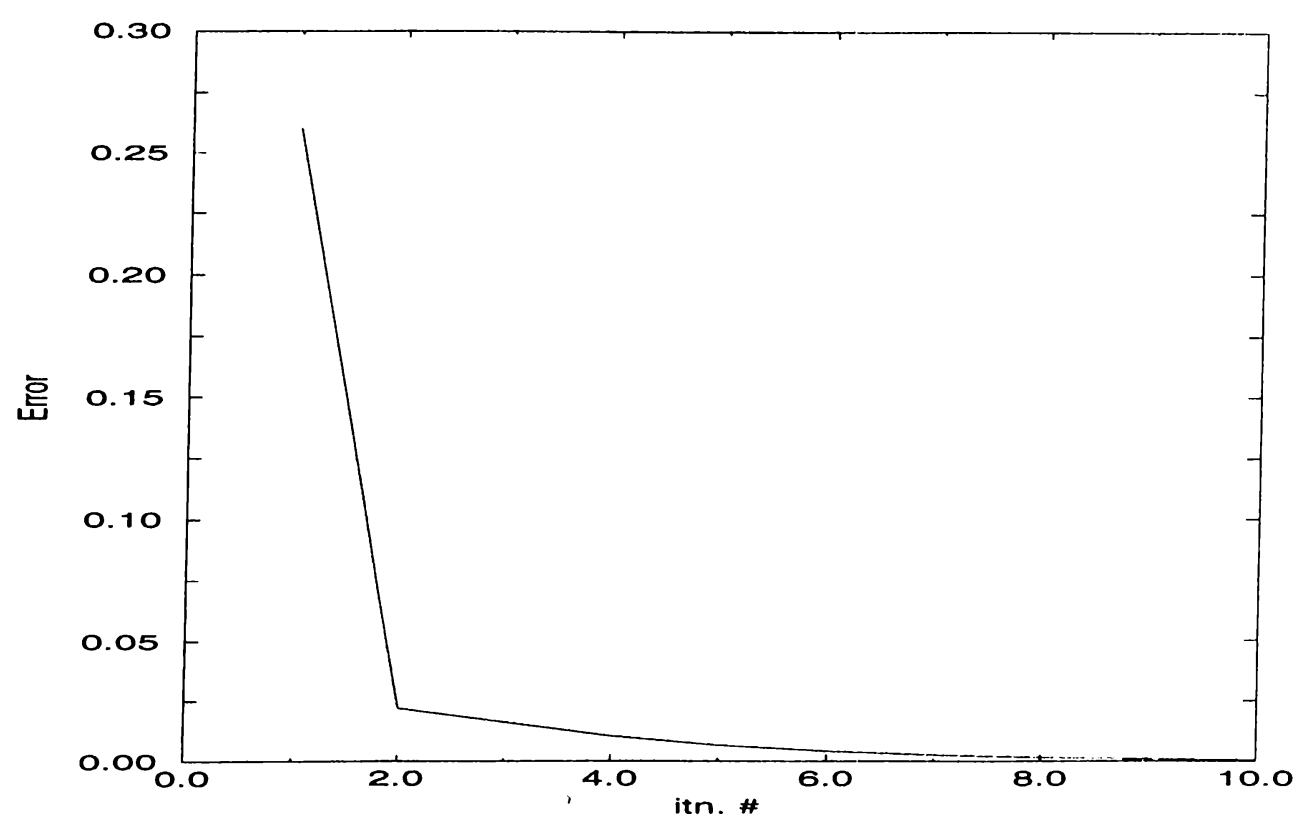

Figure 5.6: The error plot of the iterative method for the TM excitation and for $N=1000$

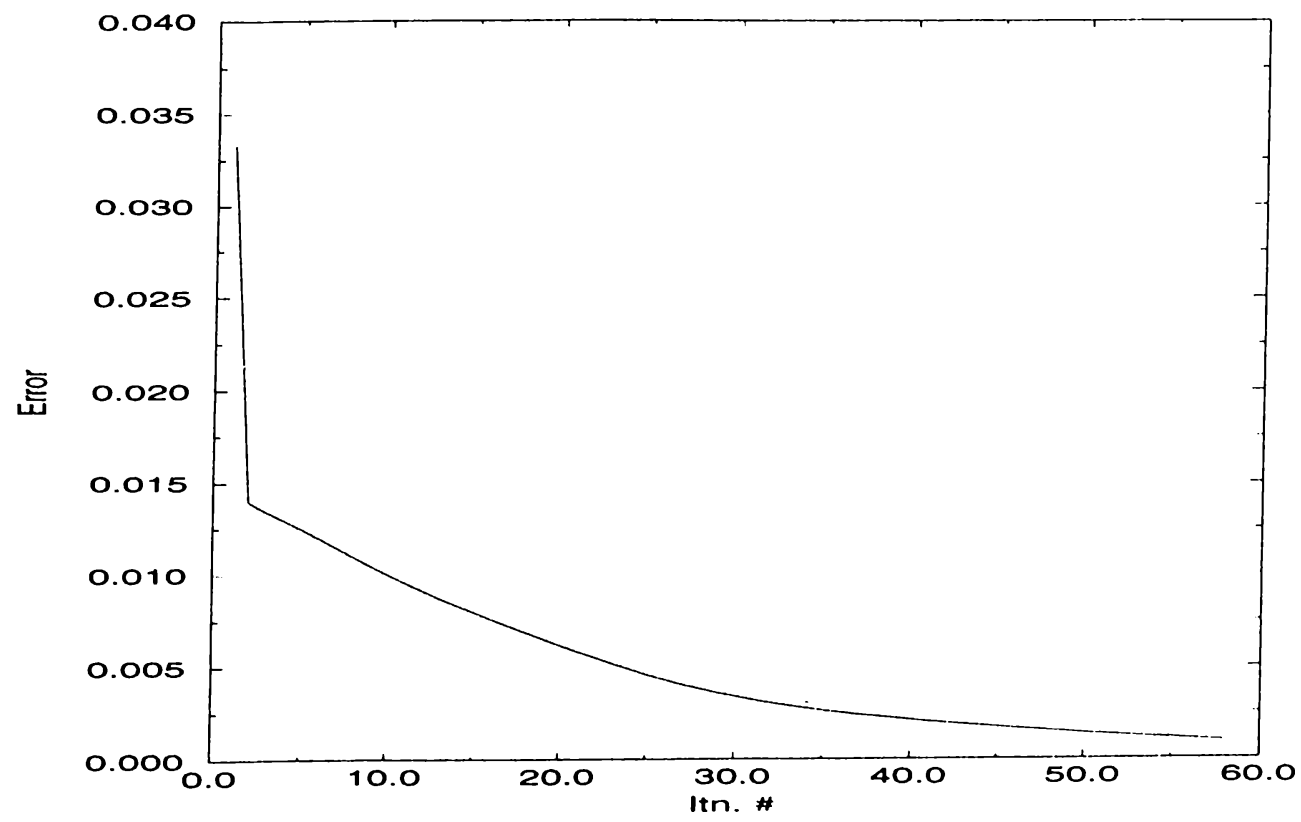

Figure 5.7: The error plot of the iterative method for the TE excitation and for $N=1000$ 


\subsubsection{Example 2}

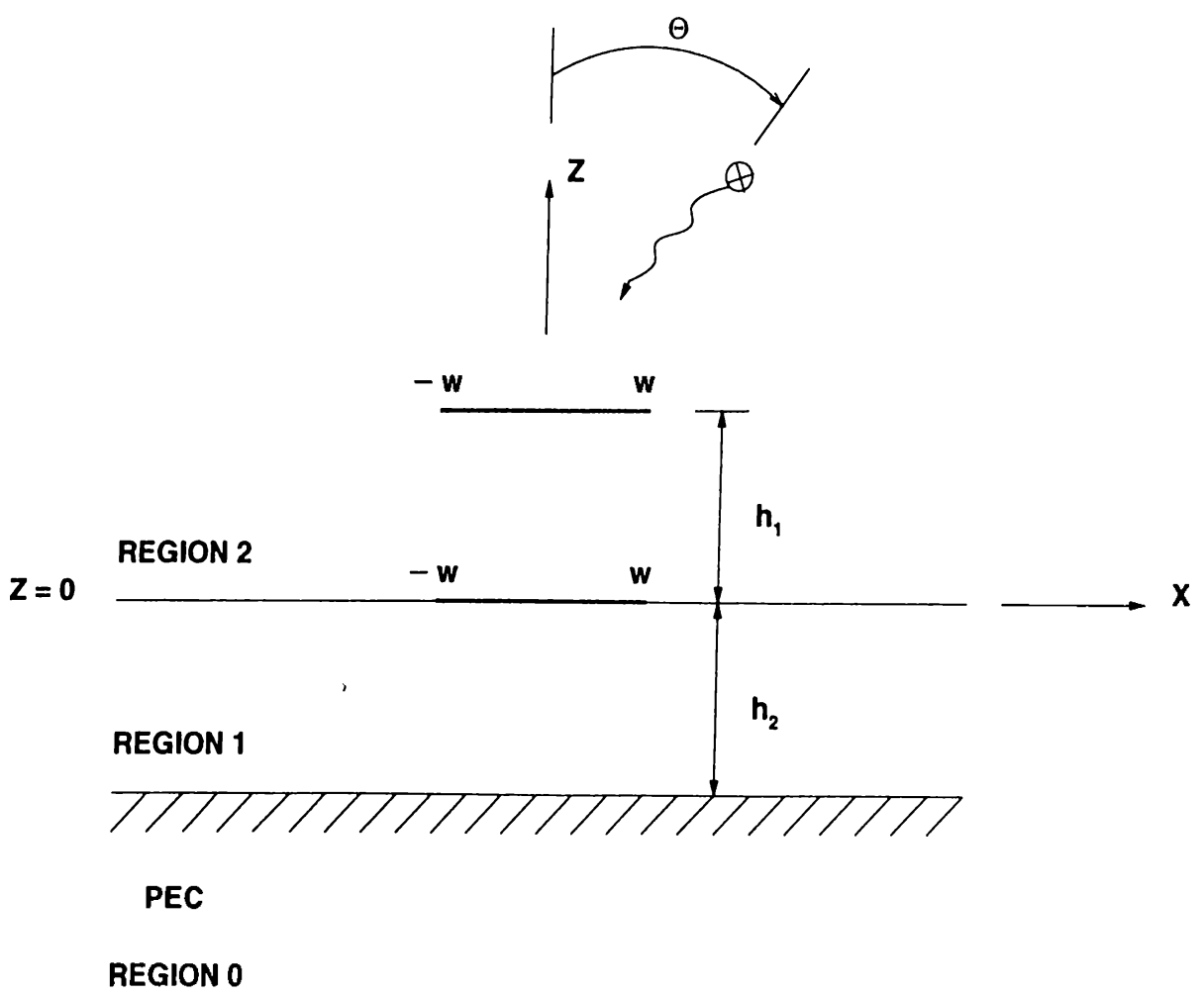

Figure 5.8: Two strips are located parallel to each other where the distance between them is $\lambda_{2}$.

The parameters of this example are as follows: Region 0 is PEC, $\epsilon_{r_{1}}=4$ and $\epsilon_{r_{2}}=1$; the width of the strips, $2 w=0.5 \lambda_{2} ; h_{1}=h_{2}=\lambda_{2} ;$ the angle of incidence, $\theta=0^{\circ}$. Table 5.6 shows $\lambda_{1}$ and $N_{2}$ which are used in the example 2 for various $N$ values. These $N_{1}$ and $N_{2}$ values are used for both the TM and TE excitation cases.

Table 5.7 and 5.8 show that when $N$ is greater than 300 , the CPU time of the iterative method is smaller than the CPU time of LU decomposition and Fig. 5.9 and 5.10 show the magnitudes of the current densities for the iterative method and LU decomposition for the TM and TE excitations, respectively. 'The results 


\begin{tabular}{||c|c|c||}
\hline $\mathrm{N}$ & $N_{1}$ & $N_{2}$ \\
\hline 100 & 10 & 10 \\
\hline 200 & 10 & 20 \\
\hline 300 & 10 & 30 \\
\hline 400 & 10 & 40 \\
\hline 500 & 10 & 50 \\
\hline 600 & 10 & 60 \\
\hline 700 & 10 & 70 \\
\hline 800 & 10 & 80 \\
\hline 900 & 10 & 90 \\
\hline 1000 & 10 & 100 \\
\hline
\end{tabular}

Table 5.6: The values of $N_{1}$ and $N_{2}$ for various $N$ for example 2.

of the iterative method and LU decomposition are found to be in good agreement. 


\begin{tabular}{||c|c|c||}
\hline \multirow{2}{*}{$\mathrm{N}$} & \multicolumn{2}{|c|}{ CPU time (s) } \\
\cline { 2 - 3 } & Ite. & LU \\
\hline 100 & 8.6 & 0.4 \\
\hline 200 & 17.5 & 3.3 \\
\hline 300 & 29.1 & 56.8 \\
\hline 400 & 43.9 & 138.9 \\
\hline 500 & 64.2 & 267.9 \\
\hline 600 & 89.6 & 453.4 \\
\hline 700 & 107.9 & 743.2 \\
\hline 800 & 134.6 & 1083.8 \\
\hline 900 & 160.2 & 1468.1 \\
\hline 1000 & 239.8 & 2460.2 \\
\hline
\end{tabular}

(a)

\begin{tabular}{||c|c||}
\hline$N$ & Iteration Number \\
\hline 100 & 9 \\
\hline 200 & 9 \\
\hline 300 & 10 \\
\hline 400 & 10 \\
\hline 500 & 11 \\
\hline 600 & 11 \\
\hline 700 & 11 \\
\hline 800 & 11 \\
\hline 900 & 11 \\
\hline 1000 & 11 \\
\hline
\end{tabular}

(b)

Table 5.7: (a) The CPU times of the iterative method and LU decomposition (b) The iteration number of the iterative method for the TM excitation and example 2 . 


\begin{tabular}{||c|c|c||}
\hline \multirow{2}{*}{$\mathrm{N}$} & \multicolumn{2}{|c|}{ CPU time (s) } \\
\cline { 2 - 3 } & Ite. & $\mathrm{LU}$ \\
\hline 100 & 12.2 & 0.6 \\
\hline 200 & 16.3 & 5.1 \\
\hline 300 & 23.1 & 59.4 \\
\hline 400 & 38.5 & 140.4 \\
\hline 500 & 68.7 & 299.7 \\
\hline 600 & 58.3 & 489.3 \\
\hline 700 & 81.8 & 780.0 \\
\hline 800 & 106.4 & 1111.2 \\
\hline 900 & 224.3 & 1621.9 \\
\hline 1000 & 350.4 & 2173.4 \\
\hline
\end{tabular}

(a)

\begin{tabular}{||c|c||}
\hline$N$ & Iteration Number \\
\hline 100 & 9 \\
\hline 200 & 9 \\
\hline 300 & 3 \\
\hline 400 & 7 \\
\hline 500 & 8 \\
\hline 600 & 3 \\
\hline 700 & 2 \\
\hline 800 & 2 \\
\hline 900 & 10 \\
\hline 1000 & 8 \\
\hline
\end{tabular}

(b)

Table 5.8: (a) The CPU times of the iterative method and LU decomposition (b) The it eration number of the iterative method for the TE excitation and example 2 . 


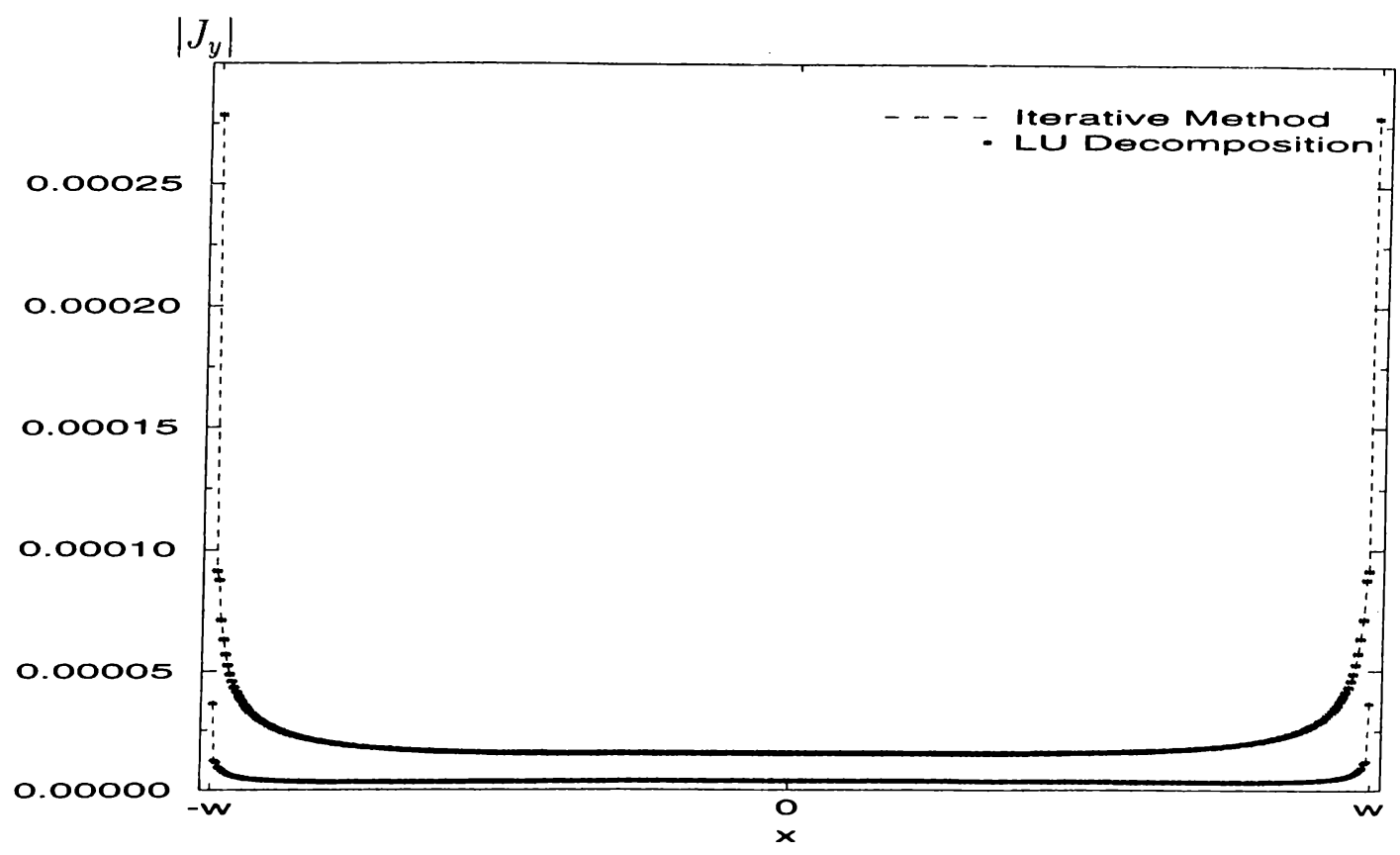

Figure 5.9: The magnitude of the current densities for the iterative method and LU decomposition for the TM excitation, $N=1000$ and example 2

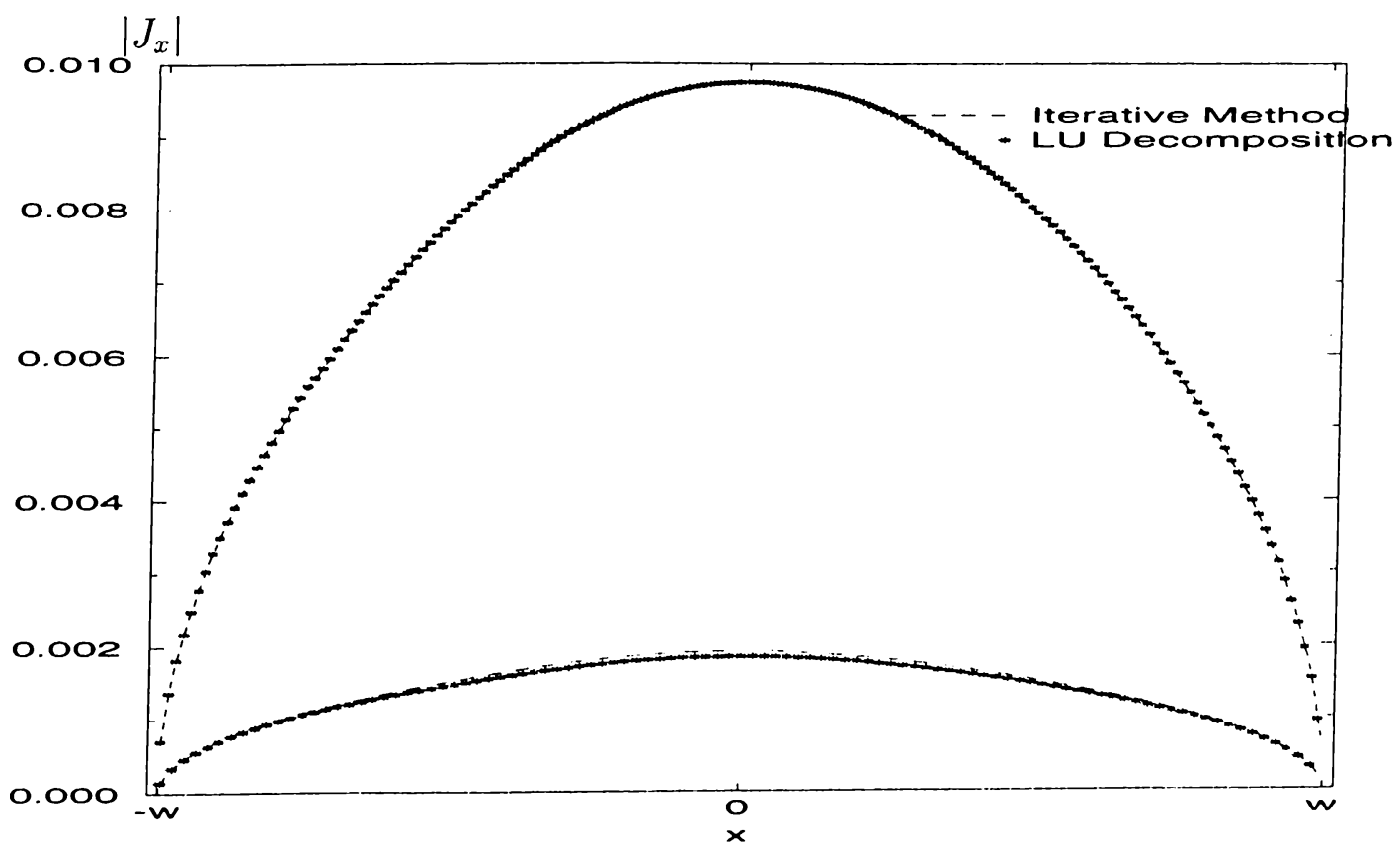

Figure 5.10: The magnitude of the current densities for the it erative method and LU decomposition for the TE excitation, $N=1000$ and example 2 


\subsubsection{Example 3}

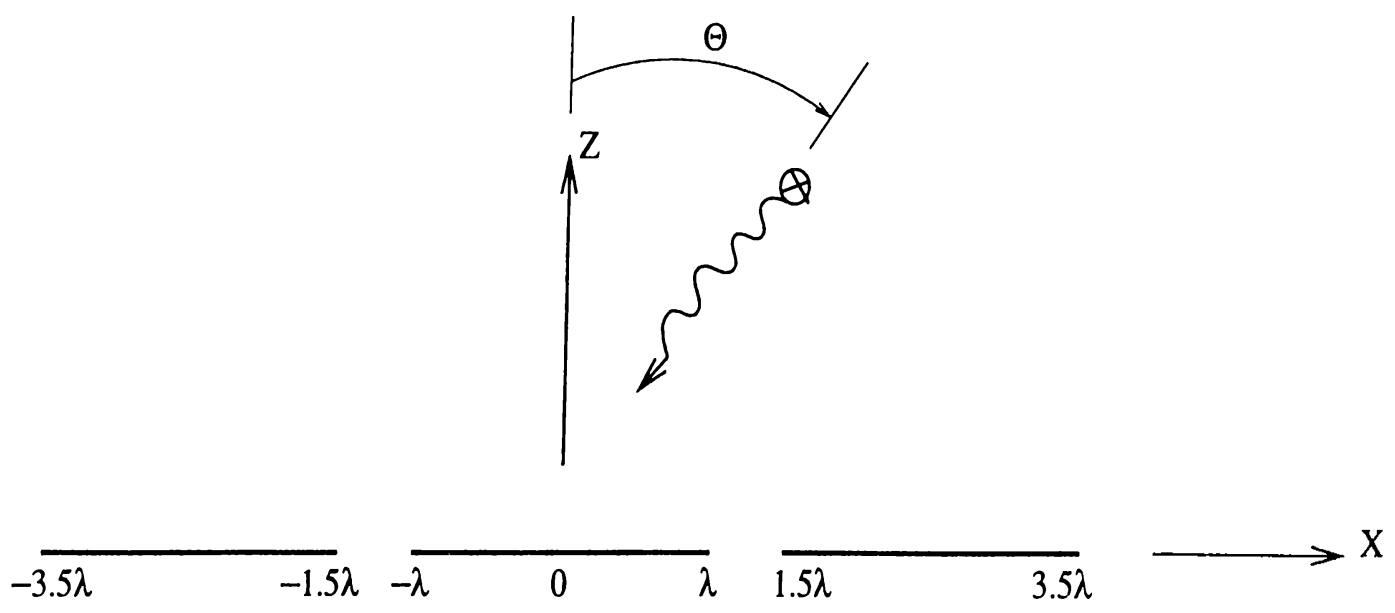

Figure 5.11: Three strips of width $2 w$ located in homogeneous medium with $0.5 w$ spacing between them on the same $z$

This example consists of three strips of $2 \lambda_{0}$ widths and $0.5 \lambda_{0}$ spacing between the strips as shown in Fig. 5.11. They are located in homogeneous medjum on the same $z$ plane. The number of unknowns for this system is taken as 900 ; $N_{2}=90$; the angle of incidence, $\theta=0^{\circ}$ and $60^{\circ}$. Table 5.9 shows the CPU times of the iterative method and $\mathrm{LU}$ decomposition and the number of iterations for the iterative method for T.M and TE excitations.

Again, the CPU time of the iterative method is observed to be smaller than the CPU time of LU decomposition since the number of unknowns is very large. Figures 5.12 - 5.15 show that the results are in good agreement for the iterative method and LU decomposition.

These examples show that when the number of unknowns of the problem is large, the CPU time to solve the linear system becomes huge when LU decomposition is used to find the solution. The use of the iterative method reduces the 


\begin{tabular}{||c|c|c|c||}
\hline \multicolumn{2}{||c||}{ CPL Time(s) TM excitation } \\
\hline \multicolumn{2}{||c||}{$\theta=0^{\circ}$} & \multicolumn{2}{|c|}{$\theta=60^{\circ}$} \\
\hline 118.5 & 1548.2 & 138.4 & 1625.1 \\
\hline
\end{tabular}

(a)

\begin{tabular}{||c|c|c|c||}
\hline \multicolumn{3}{||c|}{ CPU Time(s) TE excitation } \\
\hline \multicolumn{2}{|c||}{$\theta=0^{\circ}$} & \multicolumn{2}{c|}{$\theta=60^{\circ}$} \\
\hline 486.7 & 1573.6 & 493.1 & 1717.4 \\
\hline \multicolumn{4}{|c|}{ (b) }
\end{tabular}

\begin{tabular}{||c|c|c|c||}
\hline \multicolumn{3}{||c||}{ Iteration number } \\
\hline \multicolumn{2}{|c||}{$\mathrm{TM}$} & \multicolumn{2}{c||}{$\mathrm{TE}$} \\
\hline$\theta=0^{\circ}$ & $\theta=60^{\circ}$ & $\theta=0^{\circ}$ & $\theta=60^{\circ}$ \\
\hline $\mathbf{9}$ & 10 & 46 & 43 \\
\hline
\end{tabular}

(c)

Table 5.9: The CPU times of the iterative method and LU decomposition for (a) TM and (b) TE excitations and (c) the iteration numbers of the TM and TE excitations when $\theta$ is $0^{\circ}$ and $60^{\circ}$

CPU time hence improves the computational efficiency. 


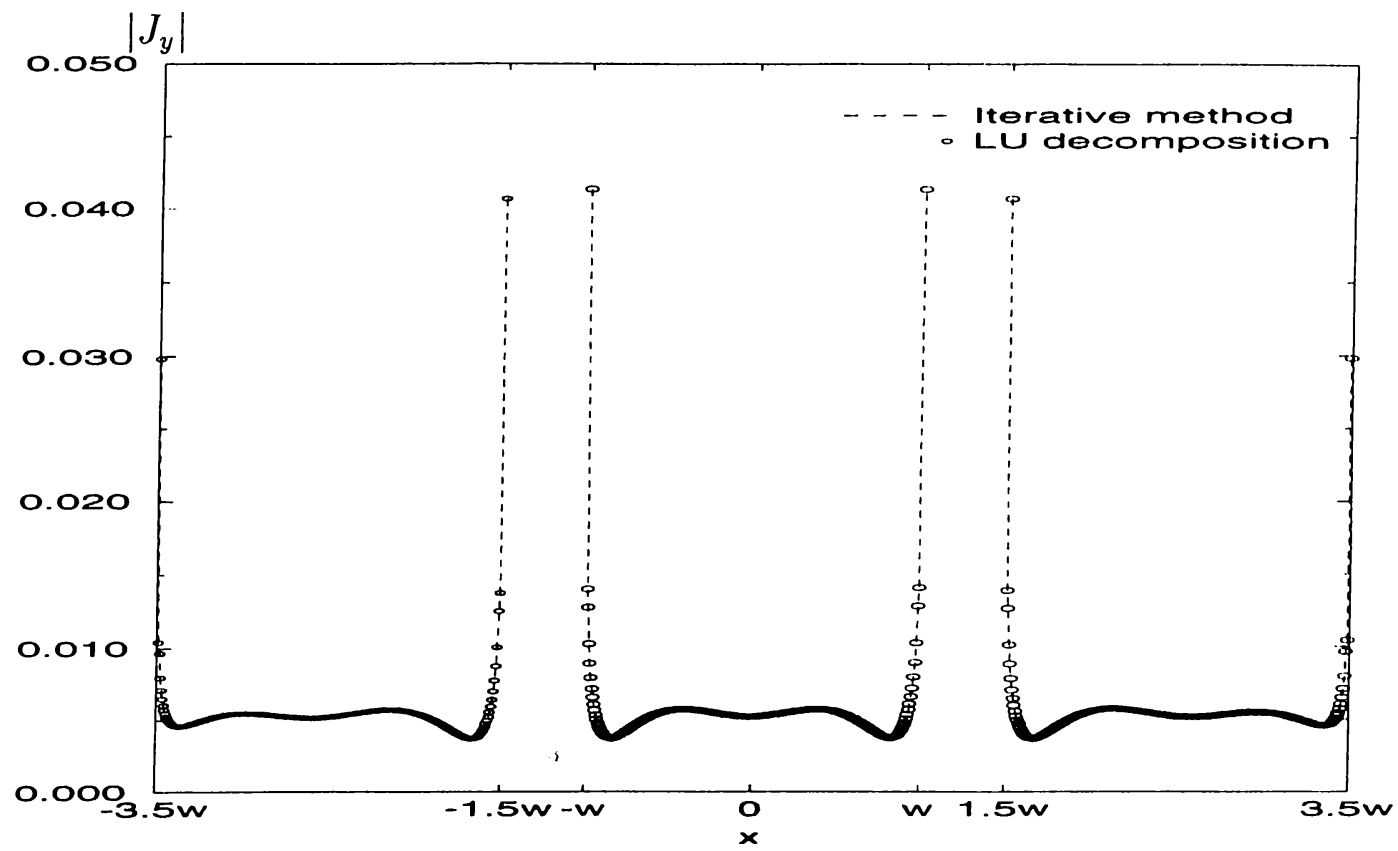

Figure 5.12: The current magnitude of the iterative method and LU decomposition for $\theta=0^{\circ}$ and TM excitation

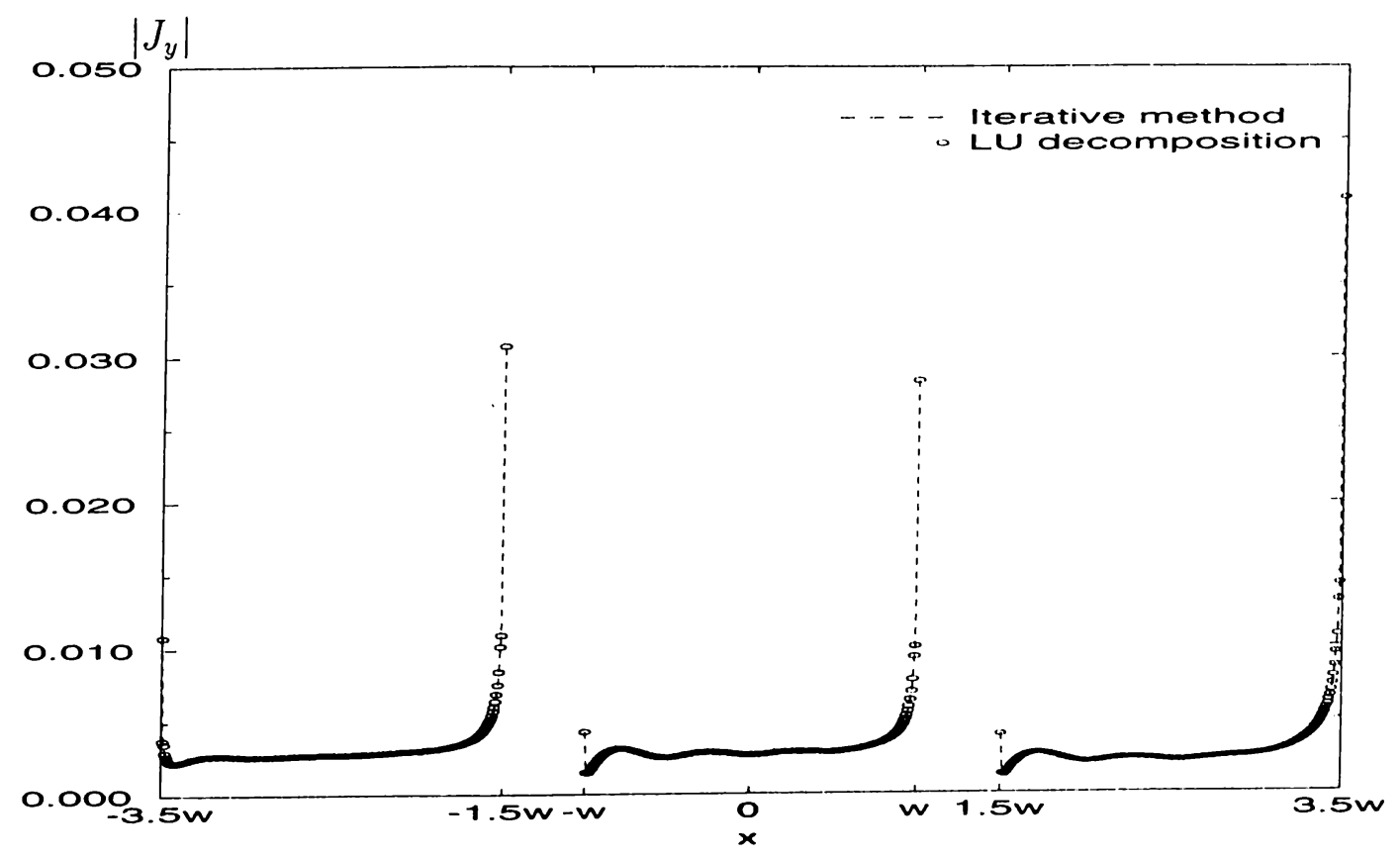

Figure 5.13: The current magnitude of the iterative method and LU decomposition for $\theta=60^{\circ}$ and TM excitation 


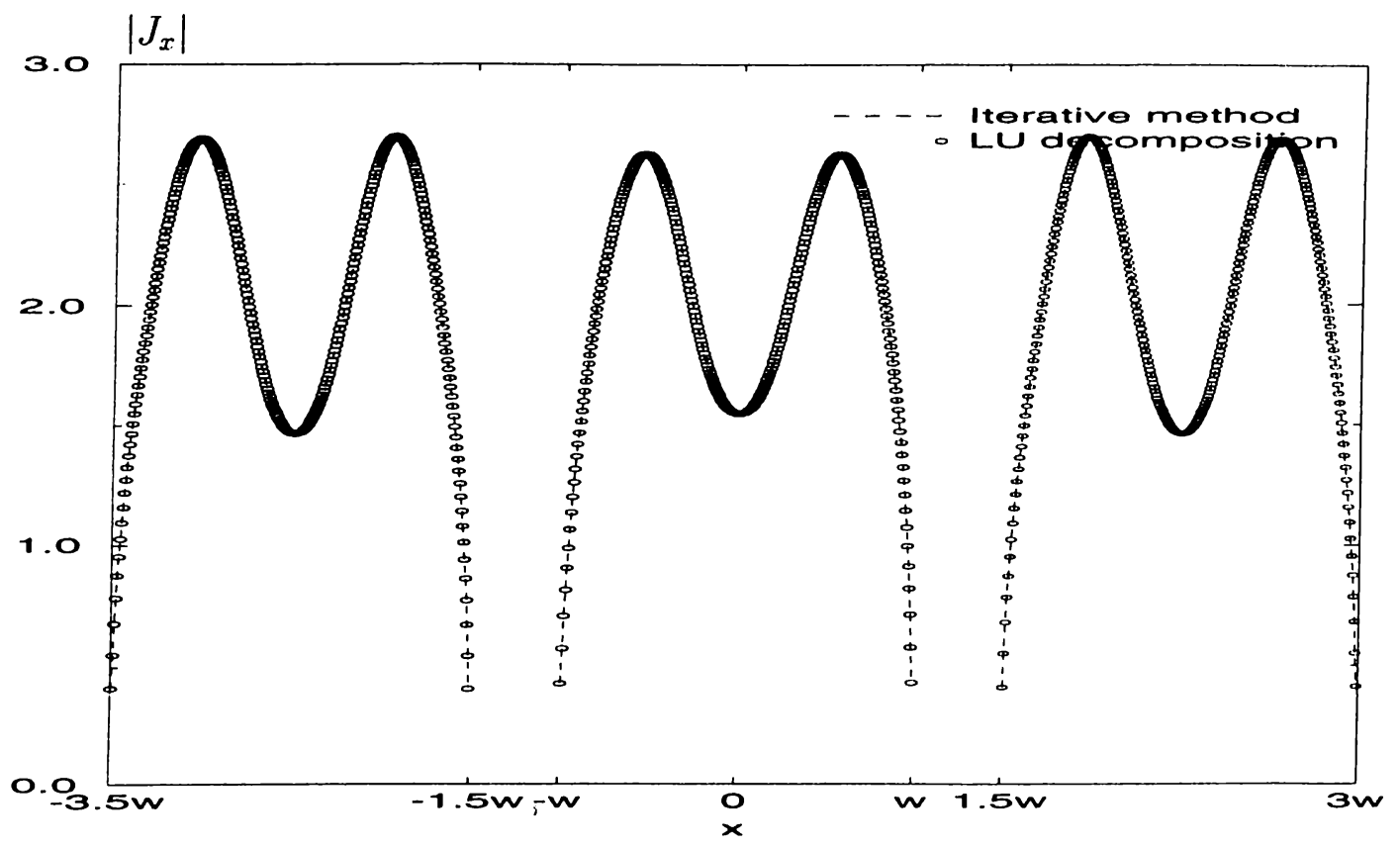

Figure 5.14: The current magnitude of the iterative method and LU decomposition for $\theta=0^{\circ}$ and TE excitation

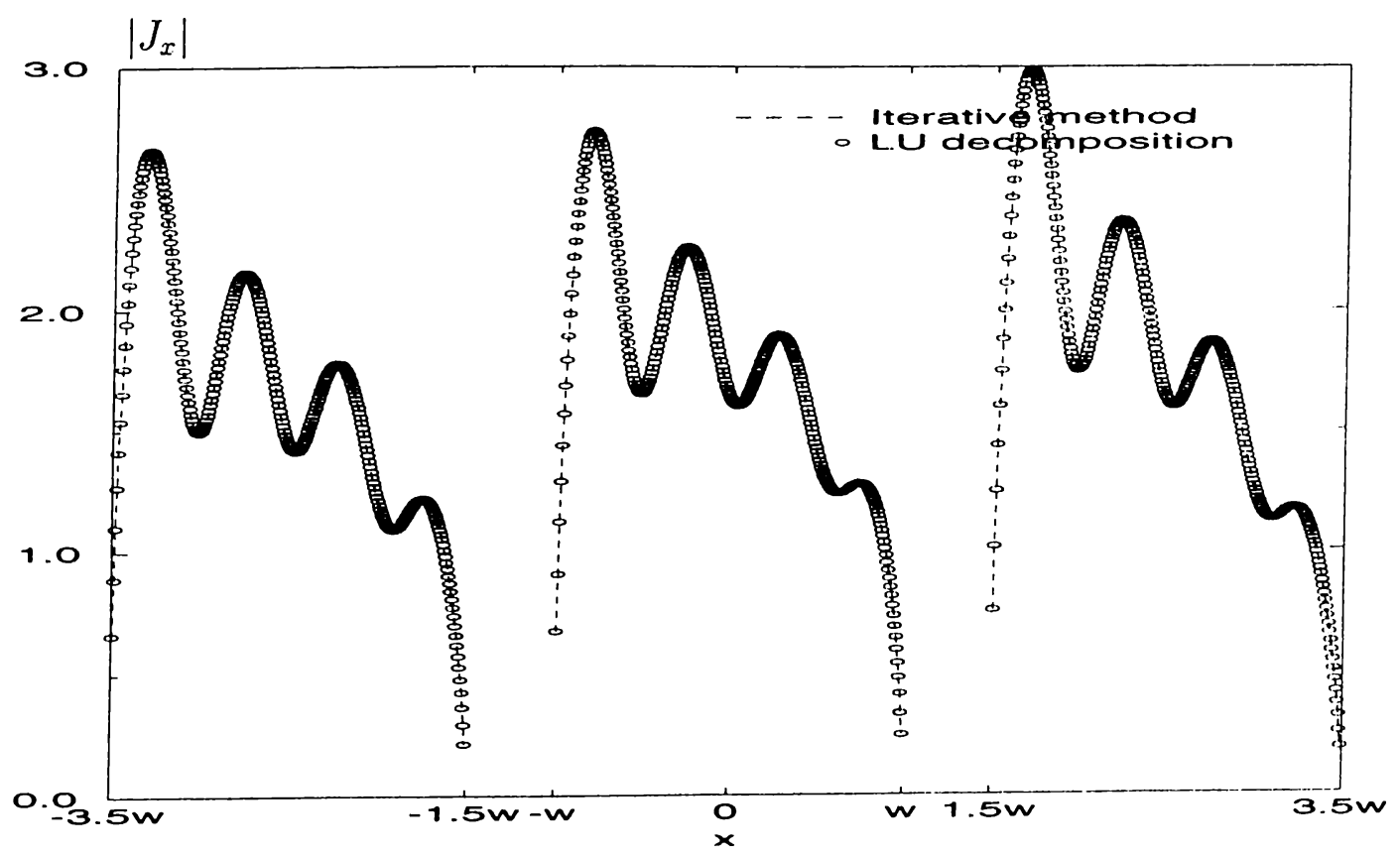

Figure 5.15: The current magnitude of the iterative method and LU decomposition for $\theta=60^{\circ}$ and $\mathrm{TE}$ excitation 


\section{Chapter 6}

\section{Conclusions}

As it is well-known, the application of the MoM to 2-D planar multilayer geometries transforms integral equations into matrix equations whose entries become double integrals over finite domains in the spatial-domain MoM, and single integrals over infinite domain in the spectral-domain MoM. In this thesis. three different algorithms to efficiently evaluate those integrals have been studied, which are namely; i) the use of closed form Green's functions for the spatial-domain MoM formulation, ii) the use of generalized pencil of functions (GPOF). and iii) the use of FFT algorithm. both in the spectral-domain MoM formulation. The first two approaches have been developed in this thesis and the results obtained for different applications are compared with each other and with the third approach. It is observed that there is no accuracy problem in any of these approaches, but as far as the numerical efficiency of these algorithms are concerned the one using the GPOF formulation is the best, which has been verified for several examples by giving the CPU times for filling the MoM matrices. 
The efficiency in the matrix fill time is important for moderate size geometries requiring a few hundred of basis functions, however for large geometries the solution of the matrix equation dominates the overall performance of MoM techniques. To improve the matrix solution time in addition to the matrix fill time, an iterative algorithm has been developed, which is based on dividing a large object into subregions and solving the matrix equation on each subregion by considering the effects of the other regions. This algorithm was compared to the conventional LU decomposition and was proven that it is superior.

As a future work, the spectral-domain MoM using the GPOF algorithm will be applied to more general geometries in multilayer media. In addition, to improve the convergence of the iterative method, overlapped subregions will be used to reduce the iteration number so that the $\mathrm{CPU}$ time required for this method will be improved. 


\section{References}

[1] Roger F. Harrington. Field Computation by Moment Methods. The Macmillan Company, 1968.

[2] Roger F. Harrington "Matrix methods for field problems," IEEE Proceedings, vol. 55, pp. 136-149, Feb. 1967.

[3] J. Jin. The Finite Element Method in Electomagnetics. Newyork: John Wiley \& Sons, Inc., 1993.

[1] K. Kunz and R. Luebber. The Finite Difference Time Domain method for Electomagnetics. Boca Raton, FL: CRC press, 1993.

[5] Juan R. Mosig "Arbitrarily Shaped Microstrip Structures and Their Analysis with a Mixed Potential Integral Equation," IEEE Trans. on Microwave Theory Tech., vol. MTT-36, pp. 314-32:3, February 1988.

[6] Ilkmo Park, R. Mittra, and M. I. Aksun "Numerically. Efficient Analysis of Planar Microstrip Configurations Using Closed-form Green`s functions," IEEE Trans. on Microwave Theory Tech., vol. 43, pp. 394-400, February 199.5. 
[7] Krishna Naishadham and Todd W. Nuteson "Efficient Analysis of Passive Microstrip Elements in MMICs," Int. J. MIMICAE, vol. 1, pp. 219-229, July 1994.

[8] S. L. Marple. Digital Spectral Analysis with Applications. Englewood Cliffs, NJ: Prentice-Hall, 1987.

[9] M. I. Aksun and Raj Mittra "Derivation of closed-form green's functions for a general microstrip geometry," IEEE Trans. on Microwave Theory Tech., vol. 40, pp. 2055-2062, Nov. 1992.

[10] Hua Yingbo and Tapan K. Sarkar "generalized pencil-of-function method for extracting poles of an em system from its transient response," IEEE Trans. on Antennas and Propagation, vol. AP-37, pp. 229-234, Feb. 1989.

[11] G. Dural and M. I. Aksun "Closed-form green's functions for general sources and stratified media," IEEE Trans. on Microwave Theory Tech., vol. 43, pp. $1545-1552$, July 1995.

[12] W. C. Chew. Waves and Fields in Inhomogeneous Media. Van Yostrad Reinhold, 1990.

[13] M. I. Aksun "A Robust Approach for the Derivation of Closed-Form Green's Functions," IEEE Trans, on Microwave Theory Tech., vol. H. pp. 6.51-6.58. May 1996.

[1-1] Alan V. Oppenheim and Roland W. Schafer. Discrete-Time Signal Processing. Prentice Hall International, Inc.. 1989. 
[15] Chalmers M. Butler "Current Induced on a Conducting Strip Which Resides on the Planar Interface Between Two Semi-Infinite Half-Spaces," IEEE Trans, on Antennas and Propagation, vol. 32, pp. 226-2:31. March 1984.

[16] Xiao-Bang Xu Chalmers M. Butler and Allen W. Gilisson "Current Induced on a Conducting Cylinder Located Near the Planar Interface Between Two Semi-Infinite Half-Spaces," IEEE Trans. on Antennas and Propagation, vol. 33, pp. 616-624, June 1985.

[17] Xiao-Bang Xu and Chalmers M. Butler "Current Induced by TE Excitation on a Conducting Cylinder Located Near the Planar Interface Between Two Semi-Infinite Half-Spaces," IEEE Trans. on Antennas and Propagation, vol. 34 , pp. 880-890, July 1986.

[18] Roger F. Harrington. Time-Harmonic Electromagnetic Fields. McGraw-Hill, 1961.

[19] Levent Gurel and W. C. Chew "A Recursive T-matrix Algorithm for Strips and Patches," Radio Science, vol. 27, pp. 387-101, May-June 1992.

[20] Samuel D. Conte and Carl de Boor. Elemenlary Numerical Analysis. McGraw-Hill, 1980. 